\title{
Placement Accuracy of External Ventricular Drain when comparing Freehand Insertion to Neuronavigation Guidance in Severe Traumatic Brain Injury
}

\author{
Ahmed Al-Azri \\ Department of Experimental Surgery \\ McGill University \\ Montreal, Canada \\ February 2016
}

"A thesis submitted to McGill University in partial fulfillment of the requirements of the degree of Master of Science (M.Sc.) in Experimental Surgery" 


\section{DEDICATION}

To my son, Marwan.

And

To the memory of my mother, Rahma. 


\section{ACKNOWLEDGMENTS}

Thanks go first to my advisor, Dr. Judith Marcoux, who supported me in this endeavor. I have always appreciated the trust she has shown in me. Thanks also to the program director of the neurosurgery residency at McGill University, Dr. Jeffrey Atkinson, who has given me the opportunity to have the time for the master study. I also thank the head of the neurosurgery department, Dr. Kevin Petrecca, who supported me to have the funding of this project, and to Mr. Kelvin Mok, the biomedical engineer at the neuronavigation unit, who was the key point for correspondence for the equipment supply, and who spared his time to help with the training of using the AxiEM navigation system. This project has been made possible by the funding provided by the Medtronic Surgical Technology Neurosurgery, which has provided the navigation system and the disposable kits plus the cost paid to the Research Institute of the Montreal University Hospital Centre.

I acknowledge the help from the traumatic brain injury (TBI) program at the Montreal General Hospital (MGH), and special thanks to the program coordinator, Ms. Metra Feyz. I also thank the neurosurgeons at the MGH for their support, in addition to Dr. Judith Marcoux, Dr. Mohammed Maleki and the former staff, Dr. Rajeet Saluja. My sincere thanks to my colleagues, the residents in the neurosurgery program at McGill University, who in their busy duties did not forget to inform me about new patients requiring ventricular drain placement. Thanks also extended to the library technician at the MGH, Mr. Daniel Lavigne, who was resourceful to me during my thesis writing. I appreciate the work done by the department of radiology at the MGH to help establishing the new scanning protocol for severe TBI, the CT EVD Protocol.

Gratefulness goes to the Government of Oman, my country, for the endless support in my mission. Finally, thanks to my wife, Khalsa, without here none of this work would have been possible. 


\section{CONTRIBUTION OF AUTHORS}

I am the principal author of this thesis. I principally worked on establishing the CT EVD Protocol, designed the recruitment process and implemented the navigation in the prospective group, conducted the chart's review, performed the statistical analyses and interpreted that data, reviewed the literature and wrote the text contained herein.

Co-authors of course assisted in this research. Dr. Judith Marcoux, my advisor, had the conception of this project and helped with the design. She also helped with data interpretation, and critically revised my thesis and provided valuable comments plus writing the French translation of the thesis abstract. The establishment of the CT EVD Protocol was made possible by the work done by Dr. Jeffrey Chankowsky and Mr. Kelvin Mok. Dr. Chankowsky was the neuroradiologist who was blinded to evaluated the catheter tip accuracy plus other radiological reports in pre and post procedural scans. Mr. Mok was a helpful resource for information regarding the AxiEM navigation system. The statistical analyses results and interpretations were reviewed and edited by the Mr. Muhammed Mullah, a PhD candidate in Biostatistics in his final year.

The authors report no conflict of interest concerning the methods used in this study or the findings specified in this paper, and have no personal financial or institutional interest in any of the materials, or devices described in this thesis. 


\begin{abstract}
External ventricular drain (EVD) placement is one of the most frequently performed neurosurgical procedures. Inaccuracies in the drain positioning and the need for multiple passes using the classic freehand insertion technique are increasingly reported in the literature. The problem is seen most frequently in the severe traumatic brain injury (TBI) population. Many proposed methods were discussed to improve the placement accuracy and none gained enough support to be implemented in EVD placement. The purpose of this study is to evaluate the use of electromagnetic neuronavigation guidance to aid EVD insertion to improve the accuracy and minimize the number of passes in severe TBI patients. The navigation was applied prospectively for all new severe TBI patients who required ventricular catheter placement over a year period, and this was compared to a retrospective cohort of severe TBI patients who had EVD inserted freehand in the preceding year. Fifty-four cases were recruited, 35 (64.8\%) had their EVD placed using the freehand technique and 19 $(35.2 \%)$ using navigation guidance. In the navigation group, the placement accuracy was as follows: $94.7 \%(18 / 19)$ achieved a grade 1 and 5.3\% (1/19) a grade 2, while none were in grade 3. In comparison, freehand placement was associated with misplacement (grade 2 and 3 ) in $42.9 \%$ of the cases $(\mathrm{P}-$ Value $=0.009)$. The number of passes was significantly lower in the navigation group with a mean of $1.16 \pm 0.38\{\mathrm{P}-$ Value $=0.018,95 \% \mathrm{CI}(-0.86,-0.09)\}$, compared to the freehand group with a mean of $1.63 \pm 0.88$. This suggests that using the navigation to guide EVD placement was associated with better accuracy and lower number of passes in challenging cases of severe TBI, which means less associated morbidities.
\end{abstract}




\section{RÉSUMÉ}

L'insertion d'un drain ventriculaire externe (DVE) externe est l'une des interventions neurochirurgicales les plus fréquemment exécutées. Le positionnement inadéquat du drain et la nécessité de recourir à de multiples passages en utilisant la technique d'insertion à main levée classique sont de plus en plus rapportés dans la littérature. Le problème est le plus fréquent dans la population avec traumatismes crânio-cérébral grave (TCC) graves. Beaucoup de méthodes proposées ont été discutées pour améliorer la précision de placement mais aucune n'a gagné suffisamment de soutien pour être mis en utilisation lors de l'insertion d'une DVE. Le but de cette étude est d'évaluer l'utilisation du guidage par neuronavigation électromagnétique pour faciliter l'insertion EVD et pour améliorer la précision et minimiser le nombre de passages dans les patients TCC graves. La navigation a été appliquée de façon prospective pour tous les nouveaux patients TCC graves qui ont nécessité l'insertion du cathéter ventriculaire au cours d'une période de une année, et cela a été comparé à une cohorte rétrospective de patients TCC graves qui avaient eu une DVE insérée à main levée l'année précédente. Cinquante-quatre cas ont été recrutés, 35 (64,8\%) ont eu leur DVE insérée avec la technique à main levée et $19(35,2 \%)$ à l'aide du guidage de navigation. Dans le groupe de la navigation, la précision de l'emplacement de la DVE a été comme suit : $94,7 \%$ (18/19) ont obtenu un grade 1 et 5.3\% (1/19) un grade 2, alors qu't aucun n'a eu de grade 3. En comparaison, l'insertion à main levée a été associée à un mauvais emplacement (grade 2 et 3 ) dans $42,9 \%$ des cas (valeur $p=0,009$ ). Le nombre de passages était significativement plus faible dans le groupe de navigation avec une moyenne de 1,16 $\pm 0,38$ $\{$ valeur $\mathrm{p}=0,018$, IC à $95 \%(-0,86,-0,09)\}$, par rapport au groupe à main levée, avec une moyenne de $1,63 \pm 0,88$. Ceci suggère que l'utilisation de la navigation pour guider le positionnement des DVE a été associée à une meilleure précision et moins de nombre de passages dans les cas difficiles de TCC grave, ce qui signifie moins de morbidités associées. 


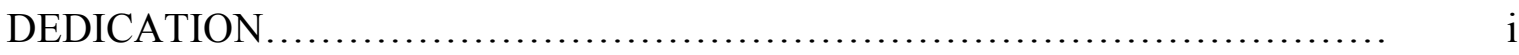

ACKNOWLEDGEMENTS .................................................. ii

CONTRIBUTION OF AUTHORS............................................. iii

ABSTRACT ...................................................................... iv

RÉSUMÉ....................................................................

TABLE OF CONTENTS..................................................... vi

LIST OF TABLES ............................................................ ix

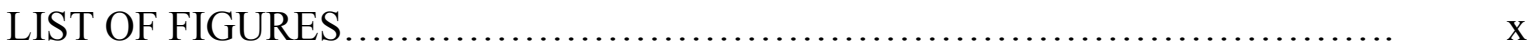

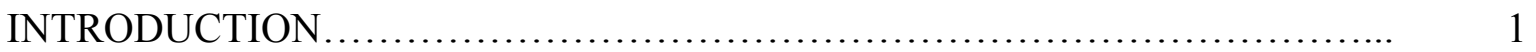

History and evolution of ventricular catheter............................. 1

Ventricular placement in traumatic brain injury.......................... 2

Intracranial pressure monitors.......................................... 4

Classic freehand EVD technique....................................... 5

Morbidity of EVD placement......................................... 6

Navigation technology in neurosurgery ................................. 8

Study hypotheses.................................................. 9

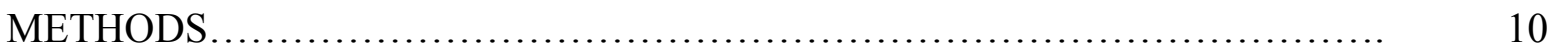

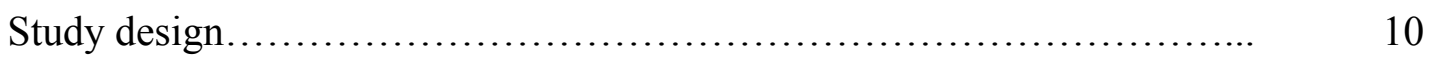

Enrollment center.................................................... 10

Patients and data collection.............................................. 11

Establishing CT EVD Protocol........................................... 12

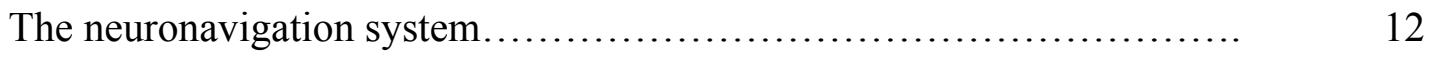

The EVD insertion procedure.......................................... 13

Classic freehand technique......................................... 13

Navigation technique............................................ 14 
Radiological evaluation.............................................. 15

Clinical evaluation................................................. 15

Statistical analysis................................................ 16

RESULTS ............................................................ 18

Baseline characteristics........................................... 18

Potential determinants of EVD placement accuracy..................... 20

Primary outcomes.............................................. 21

Clinical outcomes.................................................. 23

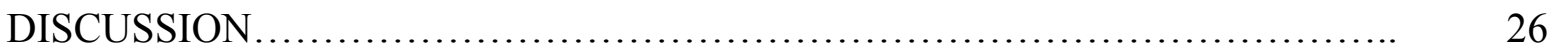

Methods reported to improve EVD placement......................... 27

Novelty of our study and the limitations............................... 28

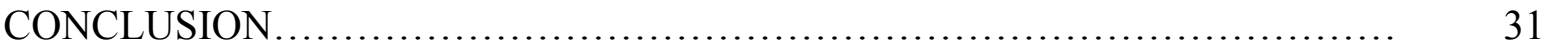

BIBLIOGRAPHY ...................................................... $\quad 32$

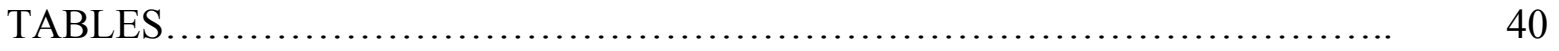

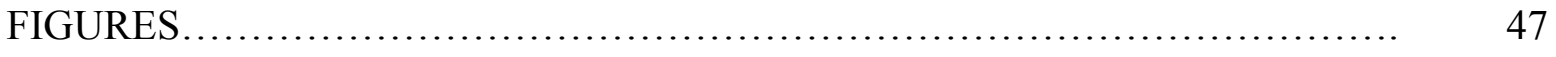

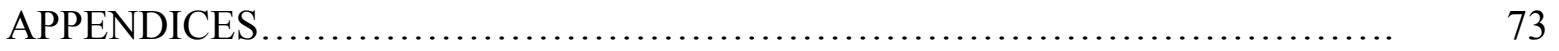

Appendix A: A table of the standard Glasgow Coma Scale (GCS) scores and

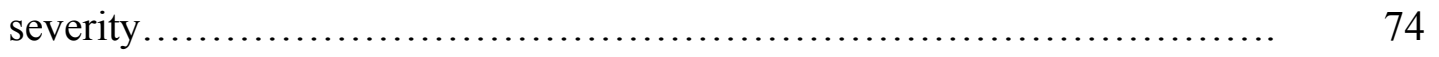

Appendix B: A table of the indications for intracranial pressure (ICP)

monitoring in severe traumatic brain injury (TBI) patients................ 75

Appendix C: A table of the Kakarla grading for the evaluation of the accuracy of ventricular catheter placement........................... 76

Appendix D: The first page of the Medtronic navigation system imaging protocol........................................................

Appendix E: The correspondence with the medical physicist at the radiology department regarding the radiation dose safety of the new CT EVD Protocol. 78 Appendix F: Electromagnetic AxiEM system equipment................. 79 Appendix G: The electromagnetic system setup registration and navigation 
highlights as been described in the Medtronic AxiEM pocket guide........ 80

Appendix H: A table of the diffuse axonal injury (DAI) grades............ 81

Appendix I: A table of the Marshall Grading for structural abnormalities in

CT scan in TBI................................................ 82

Appendix J: Correspondence to Medtronic after mid interim analysis....... 83 


\section{LIST OF TABLES}

Table 1: Summary of external ventricular drain (EVD) placement accuracy in the

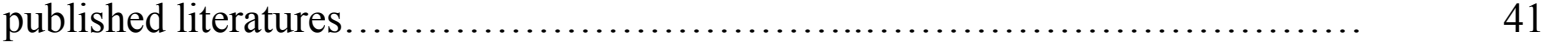

Table 2: Baseline demographics of patients............................... 42

Table 3: Potential determinants of external ventricular drain (EVD) placement.....

Table 4: Multivariate logistic regression model results of factors affecting external

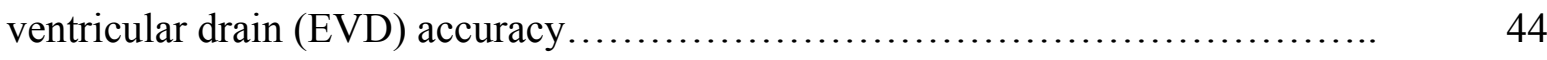

Table 5: The accuracy of external ventricular drain (EVD) procedure............. 45

Table 6: Clinical outcome of external ventricular drain (EVD) procedure........... 46 


\section{LIST OF FIGURES}

Figure 1: Different steps in applying the navigation system to guide the ventricular

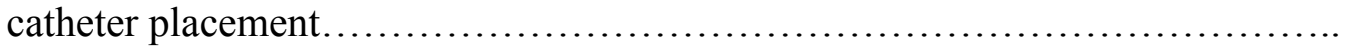

Figure 2: Different measurements applied to pre-procedural scan............... 49

Figure 3: Midline shift measurement................................... $\quad 50$

Figure 4: Catheter length using the cranium outer table..................... 51

Figure 5: The steps of recruitment................................... 52

Figure 6: Example of cases that got no intracranial pressure (ICP) pressure

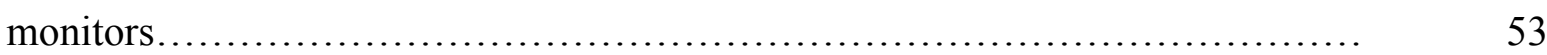

Figure 7: Different intracranial pressure (ICP) monitors...................... 54

Figure 8: Two cases that crossed over to the freehand group.................. 55

Figure 9: Bar chart showing the different intracranial pressure monitors used in

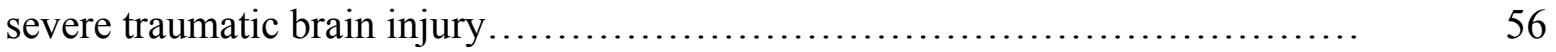

Figure 10: A challenging case managed with navigation guidance............... 57

Figure 11: Bar chart showing the mode of injury for the navigation and freehand groups

Figure 12: Bar chart showing the GCS after 24 hours for the navigation and freehand groups

Figure 13: Bar chart showing level of training and catheter placement accuracy...

Figure 14: The single case in the navigation group that had the catheter misplaced in the contralateral ventricle.

Figure 15: Example of cases with ventricular catheter tip misplacement....

Figure 16: Graph showing the number of passes related to the ventricular catheter accuracy.

Figure 17: Tract hemorrhage related to catheter placement.

Figure 18: Hemorrhagic complications and mean number of passes.

Figure 19: Bar chart of the accuracy of the entry point and safe ventricular path... 
Figure 20: Additional time required when applying the navigation guidance to the external ventricular drain (EVD) insertion procedure.........................

Figure 21: Placement accuracy relation with ventricular drain length.............. 68

Figure 22: Optimal ventricular catheter insertion............................ 69

Figure 23: Ventricular catheter misplacement detected in post-procedural scans... $\quad 70$

Figure 24: Head scan differences when using the CT EVD Protocol and routine

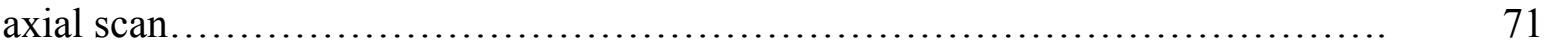

Figure 25: Example of the scanning pitfalls when used for the navigation............. 72 


\section{INTRODUCTION}

External ventricular drain (EVD) placement is one of the most commonly performed neurosurgical procedure: it is potentially lifesaving when draining hydrocephalus or when used to manage high intracranial pressure (ICP). This is often performed in the intensive care unit (ICU) and it is likely the first procedure to be learnt and performed by neurosurgery trainees on a regular basis. $(1,2)$ Optimal catheter trajectory and positioning are crucial to prevent damage to vital neuronal and vascular structures. Guidance technologies may replace the traditional blind freehand method when proven to be feasible and more accurate.

\section{History and evolution of ventricular catheter}

Alexander Monro, a Scottish anatomist, published a monograph in 1783 in which he postulated the initial Monro-Kellie hypothesis. Monro observed that the brain being enclosed in a rigid case of bone, the brain being incompressible, the volume of the blood in the cranial cavity was therefore constant, and a constant drainage of venous blood was required to make space for the continuous incoming arterial blood flow. (3) Monro's student, George Kellie, supported the observations a few years later after studying venous blood in the brain of autopsies of humans and animals that had died for various reasons. Francois Magendie added to the work done by George Burrows in the $19^{\text {th }}$ century, recognized the cerebrospinal fluid (CSF) and declared a reciprocal relationship between intracranial volume and CSF volume. (4) This added another dimension to the MonroKellie equation. Cushing, early in the $20^{\text {th }}$ century, formulated the Monro-Kellie hypothesis or doctrine and indicated that with an intact skull, the sum of the volume of the brain plus the CSF volume plus the intracranial blood volume is constant. Therefore an increase in one should cause a reduction in one or both of the remaining two. $(5,6)$ This concept is generally utilized when dealing with hydrocephalus or with increase ICP due to cerebral edema or intracranial hematoma. Hence, the ICP monitoring and CSF drainage have been conceptualized later on in the $20^{\text {th }}$ century.

The history of EVD is an example of a technical innovation and evolution in the field of neurosurgery. Many centuries ago, its utmost indication was CSF drainage in cases of hydrocephalus. Fabricius was the first to introduce cannula to drain the ventricles as he 
described in his major surgical text, Opera Chirurgica that was published in 1617. (7) Prior to it, hydrocephalus was drained using multiple skin incisions which carried high mortality rate. With evolution of the cannula as a urological instrument containing a trocar and a stopper, Claude-Nicolas Le Cat recounted its use for ventriculostomy in 1753. (8) Two centuries later, Michael Scott introduced the rubber ventricular cannula and indwelling catheter to tap or drain the ventricles, for continuous injection and pressure estimation. (9) In the second half of the $20^{\text {th }}$ centaury, other materials were used for ventricular draining such as polymer-based plastic catheters, pediatric feeding tubes, spinal needles and ventricular needle trephination system. (10) Later on, ventricular catheters kits including high-standard catheter materials closely connected to draining system and a measuring column were made available. Since infection control has been a concern in the last few decades, prophylactic antibiotics or silver-impregnated catheters were evaluated in clinical practice. $(1,11-13)$ The accuracy of ventricular catheter placement and associated morbidities has come to attention also in the last few decades. Different techniques were described to improve ventricular catheter placement. The application of navigation-guidance was evaluated for ventricular shunt placement in general and the EVD placement in particular. $(1,14,15)$

\section{Ventricular placement in traumatic brain injury}

Historically, the first and ultimate indication for ventriculostomy was CSF drainage in cases of hydrocephalus until the second half of the $20^{\text {th }}$ century when other clinical indications came gradually into routine practice. One of these indications was in the management of traumatic brain injuries (TBI).

Severe TBI is currently one of the most common indications for EVD placement for intracranial pressure monitoring and simultaneously cerebrospinal fluid (CSF) drainage to control the ICP. $(1,16)$ TBI is a major health and socioeconomic problem globally, and the most common cause of death and disability in young people. (17-19) According to the Centers for Disease Control and Prevention in USA, motor vehicle traffic crashes account for the majority of TBI-related deaths in youth and young adults, whereas falls account for the majority of deaths in adults 65 years of age and older. (20) It is clear that only part of the damage to the brain occurs at the moment of impact. Secondary insults are blamed for significant part of brain injuries. In history, the death of the American President Abraham Lincoln in 1865 who was assassinated by a gun shot to the back of his head, 
retrospectively, highlighted the role of intracranial pressure and CSF drainage in a well documented clinical progress until the time of his death in the consequent hours. Mechanical clots removal from the accidental ventriculostomy track allowed CSF and blood drainage, which were resulted in transient improvement of his respiration. (21) The Glasgow Coma Scale (GCS) was introduced in 1974, aiming at standardizing assessment of level of consciousness in head injury patients. It includes three aspects of behavioral responses, namely eye opening, verbal and motors responses. $(22,23)$ Nowadays, it is by far the most widely used score to assess the severity of TBI patients in clinical practice and in research to compare series of patients. The classification of mild, moderate and severe TBI is based mostly on the GCS of the injured person as assessed in the emergency department. Score 13-15 is classified as mild, 9-12 as moderate, and 3-8 as severe (Appendix A).

Marshall et al.'s report in 1979 was probably the first to highlight the significance of ICP monitoring in severe TBI. (24) Intensive management protocols implementation since the late 1970s was reported to significantly reduce mortality and morbidity of severe TBI. The management of elevated ICP is important to prevent secondary brain injury, and uncontrolled high ICP is associated with mortality and worse functional outcome in severe TBI. $(16,24-29)$ The high ICP is difficult to be reliably predicted by computed tomography (CT) scan. Treating high ICP without having the ICP monitored to guide the therapy may result in poor outcome. The Brain Trauma Foundation review supported the use of ICP monitoring in severe TBI to guide therapy and improve outcome. (16) The indications for EVD placement in general include all severe TBI. As per the recommendation of the Brain Trauma Foundation these indications include salvageable patients with GCS score of 3-8 after resuscitation and abnormal brain CT scan. In case of normal scan, then EVD may be placed if two or more of the following criteria are present: age more than 40 years, motor posturing, and systolic blood pressure less than $90 \mathrm{mmHg}$ (Appendix B). (16) Controversially, some reports critiqued the approach of maintaining management of severe TBI based on ICP monitoring targets (30-33), and a controlled trial by Chesnut et al. found that care focused on maintaining ICP at $20 \mathrm{~mm} \mathrm{Hg}$ or less was not shown to be superior to care based on imaging and clinical examination. (34) However, none of these studies argued against the use of ICP monitoring in severe TBI, and probably there is only a subset of this population who may not benefit from ICP monitoring. 


\section{Intracranial pressure monitors}

The optimal ICP monitoring device is one that is accurate, reliable and cost effective with minimal possible complications. There are multiple techniques: invasive as well as noninvasive. There are different types of monitors with different technologies of pressure transduction and different insertion targets in the intracranial compartment. Examples of pressure transduction methods are fluid-coupled external strain gauge and micro strain gauge, and fiberoptic and pneumatic monitors. The targeted compartments that are commonly used are ventricular space and intraparenchymal, whereas subarachnoid, subdural and epidural locations are rarely used.

Historically, ventricular pressure measurement is used as the reference standard for ICP monitoring, and ventricular-placed catheters can give more generalized intracranial pressure compared to the monitors placed in other locations. A ventricular catheter connected to an external strain gauge transducer, i.e. EVD, is the most accurate, reliable and cost effective method for monitoring ICP. (35) Additional advantage to this method, apart from measuring the ICP, are: the possibility to drain CSF when required to control ICP and collect CSF samples for investigations, and the ability to be recalibrated in situ. Obstruction of the fluid couple can cause inaccuracy and to avoid measurement errors, it must be consistently maintained at a fixed reference point relative to the patient's head and the most used point is the external auditory meatus. Other potential risks of catheter insertion, misplacement, infection and hemorrhage have led to the development of alternative ICP monitoring methods. (35)

Parenchymal pressure monitors may have less complication rates compared to the ventricular catheters, though this might not entail significant clinical sequelae. (36) The negative side about the parenchymal monitors is that they have the tendency to develop measurement drifts, and once inserted they cannot be recalibrated; consequently, this may result in inaccurate measurement. This method is expensive, since it includes the cost of microsensor pressure monitoring kit plus the reusable display monitor and calibration device. (35) In a prospective observation study by Liu et al., using EVD was superior for controlling refractory ICP with higher post injury survival rate. (37) In severe TBI, parenchymal ICP monitors can be used in selective cases where the scan is not supportive of high ICP to explain patient's depressed neurological status. However, the risk of drift without the possibility to calibrate shall be kept in consideration. In most of the cases, EVD is still advocated for ICP monitoring, in addition to its benefit to drain CSF to 
manage ICP or to have diagnostic sampling. In TBI population, parenchymal monitors are typically being used when EVD fails placement, on in cases of moderate TBI for which frequent clinical evaluation is not feasible. For example when patients are subjected to long sedation or anesthesia time in significant multisystem trauma.

\section{Classic freehand EVD technique}

Classically, EVD placement is a blind procedure that is performed using a freehand technique through a frontal approach using anatomical surface landmarks at bedside in ICU. Neurosurgery residents or trainees are supervised for the first few initial cases of EVD insertion by a faculty or a senior resident. Most residents become proficient very early in their training. The resident would understand the proper techniques and get the sense of the procedure before performing it on a patient. The procedure involves placing ventricular catheter at the ipsilateral ventricle close to the foramen of Monro. There is considerable variation in the precise landmarks used to place EVD. A craniostomy is performed at Kocher's point, which is $10 \mathrm{~mm}$ anterior to the coronal suture, approximately $10 \mathrm{~cm}$ above the nasion, in the mid-pupillary line about $2.5-3.0 \mathrm{~cm}$ lateral to midline. $(38,39)$ Friedman and Varies described the coronal plane to be at the coronal suture. (40) The direction of the hole is placed perpendicular to the cranium, making it virtually pointing medially and posteriorly. After puncturing the dura, the ventricular catheter is advanced targeting the frontal horn of the ipsilateral ventricle. The catheter is slid down in angulation, directly targeting the intersection of virtual lines between the ipsilateral medial canthus sagitally and the external auditory meatus or tragus coronally. Muirhead et al. had found that using the sagittal plane through the ipsilateral medial canthus was a poor trajectory, and using the contralateral medial canthus with the external auditory meatus trajectory was a more reliable way of targeting EVD placement. (39) Another popular method is to direct the catheter perpendicular to the scalp and skull surface with less care about the virtuality of the target. Ghajar Guide device to facilitate catheter placement was developed on this second principle and works by directing the catheter directly perpendicular to the skull surface. $(41,42)$ In a survey conducted to characterize different surgical techniques for targeting lateral ventricles, the average ventricular catheter length to the outer table of the skull ranged between $5.0-7.75 \mathrm{~cm}$, with the median length of $6.0 \mathrm{~cm}$. Setting this length limit may minimize EVD misplacement in deep vital structures. $(43,44)$ On occasions, a pop can be felt on 
puncturing the ventricle, and dripping of CSF should be seen indicating EVD placement within the ventricle. A drain that is misplaced deep in the $4^{\text {th }}$ ventricle or extra ventricular in subarachnoid spaces can still drain CSF; however, the reliability as ICP monitor and the efficiency for CSF drainage are questioned. There are also risks involved when passing the drain through vital brain structures during insertion in addition to risking vascular injuries. (45-49)

\section{Morbidity of EVD placement}

Inaccuracy of EVD placement using the freehand technique has been increasingly reported in the literature (Table 1). Thirty years ago, this was brought to attention by Ghajar who published his technique using the Ghajar Device (Neurodynamics, Inc., New York, USA) to aid accurate EVD placement, after reports about the EVD misplacement in either cerebral matter or subarachnoid space. (41) Although EVD insertion is a low morbidity procedure, number of passes indicates unnecessary brain insult with each additional pass; hence, many reports included the number of passes as morbidities. Phillips et al. in a retrospective study reported that multiple passes occurred in $28 \%$ of EVD placed using freehand technique with an average of 1.85 per procedure. (50) In a mixed cohort of EVD insertions containing TBI as an indication in 50\%, Huyette et al. reported the misplacement of the drain using the freehand method to be about $40 \%$ and more importantly $20.4 \%$ of the catheter tips were within extra ventricular spaces. The number of passes was described in procedure notes in about $30 \%$ of the cases with an average of 2.17 (range 1-5) insertions per a successful EVD. (51) Toma et al. reported the misplacement within the brain parenchyma, subarachnoid space and contralateral side to be $23 \%$ in a cohort mostly of subarachnoid hemorrhage (SAH) and intracerebral hemorrhage ( $\mathrm{ICH})$. Of the misplaced ones, $40 \%$ required revision or reinsertion. It was observed that Evan's index (52), also called hydrocephalus ratio, of less than 0.4, which reflects smaller ventricular size, was associated with more chance of misplacement into the parenchyma or other CSF spaces. (53) A similar result of misplacement (23\%) was reported by Lee et al. in a Korean cohort that was mostly composed of SAH and ICH as indications for EVD insertion. (54) Hsieh et al. reported misplacement to be more than 28 $\%$ in a Chinese cohort, of which TBI constituted about $30 \%$. The misplacement rate was significantly higher in patients whose head CT scans revealed lower hydrocephalus ratio and smaller ventricular size. (55) Abdoh et al. reported $24 \%$ misplacement using the 
freehand technique and observed that inserting the tube more than $7 \mathrm{~cm}$ had more tendencies to cross the midline. (56)

There was no consensus about a grading system to evaluate EVD accuracy until Kakarla et al.'s review of ventricular catheter misplacement in 2008, in which they proposed a grading system to evaluate catheter tip location (Appendix C). (2) It is composed of 3 grades in an order of possible morbidities associated with EVD placement. Grade 1 represented optimal placement and considered the ideal target with the tip in the ipsilateral frontal horn or third ventricle through the foramen of Monro. Grade 2 represented functional placement into the contralateral lateral ventricle or noneloquent cortex. Grade 3 represented suboptimal placement into eloquent cortex or nontarget nonventricular CSF spaces. The overall accuracy (grade 1) was $76.9 \%$. In the traumatic case, which represented $18 \%$ of the studied population, the EVD placement accuracy was $56.3 \%$, in comparison to the SAH group, which represented $44 \%$ of the studied population, and in which the placement accuracy was 85.6\%. (2) Park et al. utilized Kakarla's grading to evaluate a heterogeneous group of patient mostly with SAH and ICH and found accuracy to be $81 \%$ when Kocher's point was used for entry point with overall accuracy of $88 \%$. (57) Foreman et al. compared the complications of EVD insertion in regards to the site of placement: ICU or operating room (OR). The overall accuracy of placement was found to be $63.8 \%$ with no statistical difference when the EVD was placement in the ICU or the OR. (58)

In a mixed cohort of ventriculostomy reported by Saladino et al., including EVDs (79.7\%), and ventriculoperitoneal and ventriculoatrial shunts $(20.3 \%)$ where $5.1 \%$ are post TBI, the misplacement (grade 3) was reported to be $12.3 \%$. (59) Ventricular catheter placement for acute hydrocephalus was reported to have $11.0 \%$ misplacement by Bogdahn et al.; however, the diagnosis of misplacement was not clearly addressed in the content of the study. (60) In a retrospective cohort comparing ventricular catheter placement for CSF shunting, either ventriculoperitoneal, ventriculopleural or ventriculoatrial comparing freehand, stereotactic-guided and ultrasound-guided, the only risk factor identified for placement inaccuracy was the use of freehand technique. (61) In pediatric population including TBI- related indication for ventricular catheter placement in $36 \%$ of the cases, Ngo et al. reported $6.3 \%$ misplacement into eloquent brain regions. (62) Similarly, in a pediatric population whom had EVD placed for severe TBI, the risk of misplacement reported by Anderson et al. was $8.8 \%$. (36) 
To know whether misplacement or multiple passes are deleterious to the brain, it is important to have a definition for brain damage. Imaging can detect what is not detected in routine neurological evaluation. Even detailed neuropsychological exam may miss damage evident on imaging. (63) Perhaps one of the famous brain injuries in history was the case of Phineas Gage who suffered a penetrating wound and loss of his frontal lobe. Remarkably, Gage not only survived but also apparently never fully lost consciousness or have overt neurological deficit. The most marked changes seem to have been in executive functions and personality changes; he became impulsive and disorganized and was never able to hold a position for a long while. $(64,65)$ In addition to the difficulty of diagnosing subtle damage to frontal lobes, in this group of patients who are subjected to insertion of an EVD, there is nearly always a coexisting premorbid condition making it quite impossible to detect specific neurocognitive damage. In a fraction of a percentage of EVD misplacement, the misplacement and concurrent multiple passes into eloquent brain structures can have obvious damage and consequent neurological deficits or necessitate further interventions. (45-49)

\section{Navigation technology in neurosurgery}

Computer-assisted navigation is a computer-aided and image guided stereotactic system used for surgical procedures. This technology has mainly been applied to aid anatomical and functional localization during neurosurgical procedures, especially tumor resections and functional and epilepsy surgeries. The central part of a navigation system is a tracking system. A tracking system consists of one or several sensors attached to a surgical instrument or an ultrasound probe that are tracked by a device calculating the position and orientation of the sensors. There are four different technologies to track medical instruments: mechanical, acoustic, electromagnetic, and optical. (66) Navigation using optical tracking systems is the most widely used image guidance technique in neurosurgical field. The limitations due to its complex set-up, the space required in the operating room and the need for rigid skull fixation do not favor its use for EVD insertion procedure at bed side in ICU. Electromagnetic tracking system has a transmitter that generates a magnetic field. The induced electrical current in a sensor is measured. Because the distribution of the magnetic field is known, the position and orientation of the sensor can be computed. Electromagnetic technology allows accurate and safe neuronavigation without the need for head fixation or a direct line of sight between 
tracker and probe. This technology is noninvasive, pinless (requires no head fixation), and frameless. It has an easier setup, in addition to allowing freedom of head movement without loss of accuracy of registration or interference with the surgical field. (67-70) These advantages make it applicable to be used for urgent procedure for EVD placement at bedside in ICU.

\section{Study hypotheses}

In our study, we hypothesized that the use of neuronavigation to guide EVD placement in severe TBI patients will improve the accuracy and minimize the number of catheter passes required for successful placement. The targeted population of severe TBI for this study was based on the notion that this population has more unfavorable features such as young age with small ventricles, in addition to brain edema and the resultant ventricular compartment effacement. Other unfavorable characteristics that make EVD placement more challenging in this population are the associated brain shift and anatomical distortion, and in some cases the presence of external cranial swelling or lacerations. The above-mentioned review that highlighted the risk of misplacement in TBI population supported this perception of challenging catheter placement in severe TBI. 


\section{METHODS}

\section{Study design}

This was a prospective study to evaluate the accuracy of EVD tip placed using the electromagnetic navigation system (navigation-guidance group) and comparing the results with a retrospective cohort where the classic freehand technique was used (freehand group).

The study duration was initially set to be from July 2013 until June 2014 for the retrospective freehand group, and prospectively from July 2014 until June 2015 for the navigation guidance group. However, the later was shifted 2 months forward because of the delay in instruments supply required for the navigation system.

The primary outcome was to measure the accuracy of catheter tip placement using the navigation guidance, compared to the freehand technique. Kakarla's grading was used for this evaluation (Appendix C). The other main outcome was to evaluate the multiple passes associated with each procedure. Secondary outcomes included rates of revision, infection and complications, the length of hospital stay, in addition to evaluating the extra time added by the navigation set-up and registration for the navigation group.

Approval of this study was granted by the Research Ethics Boards (REBs) of the McGill University Health Centre (MUHC) concomitant with the approval obtained from the hospital administration.

The Medtronic of Canada Ltd covered funding to provide the StealthStation AxiEM system plus the disposable kits with each containing a registration probe, a patient reference device and a sterile navigation stylet.

\section{Enrollment center}

The study was conducted at the Montreal General Hospital (MGH), which is one of the two level-1 trauma centers for adults in the region. The MGH receives a high volume of patients with trauma, and has 24-hours trauma service coverage; specialized trauma teams, ICUs specialists, and emergency computer tomographic (CT) service. The neurosurgery coverage at the $\mathrm{MGH}$ is specialized for TBI. Other urgent neurosurgery cases that are non-traumatic, after stabilization, are transferred to the Montreal 
Neurological Hospital (MNH). Traumatic brain injury team also includes specialized physiatrists, occupational therapists, physiotherapists, speech therapists, social workers and a neuropsychologist plus the program coordinator.

\section{Patients and data collection}

Data about admitted cases with TBI including their demographics, cause of injury and severity, ICP monitors and outcome are regularly updated in the TBI database at MGH. This database was the basis for enrollment of the retrospective cases in our study. Chart review was the main source of data gathering for both groups. The data included, but not limited to, patient age, GCS, ICU and hospital length of stay, the need for surgical decompression, the level of training of the person performing the procedure, the documented number of EVD passes, and the outcome.

The recruitment in the prospective group was managed as case by case. Patients with significant TBI were subjected to head CT scan as a standard of care. According to the discretion of the neurosurgery team, decision about EVD placement was made after evaluating the subjects with severe TBI. The investigator was present after decision was made to place EVD, to setup the navigation system and supervise the placement procedure.

The population of our study included all admitted adult patients ( $\geq 18$ years old) with severe TBI who required EVD insertion according to the recommendations of the Brain Trauma Foundation (Appendix B). (16)

The exclusion criteria were the ones that precluded these patients from having EVD inserted at first instance. In general those were the patients who had uncorrectable coagulopathy or who were subjected to emergency cranial surgery and got their EVD placed intraoperatively. Others who were also excluded were patients who were planned for no EVD placement according to the discretion of the treating team. Examples were patients who had guarded clinical outcome. The cases that were having no CT scan post EVD procedure were also excluded. In the prospective navigation-guidance group, candidates should have their head scanned using the CT EVD Protocol to be able to upload their scans in the navigation system. The cases that were managed with the parenchymal ICP monitors (Codman microsensor ICP transducer) were not included in our study. The EVD placement procedure is usually an urgent procedure. In traumatic 
injuries, the patient is always in a state of coma, and often no family is present; hence, the consent cannot realistically be obtained in routine practice. The need for consent for the EVD placement in our study was waved after consulting the REBs and obtaining the approval for the waiver.

\section{Establishing CT EVD Protocol}

Patients enrolled in the study are not subjected to any extra CT scans. The establishment of CT EVD Protocol at the MGH emergency CT scanner (GE, LightSpeed VCT 64 Slices) eliminated the need for an additional scan to meet the navigation imaging protocols (Appendix D).

Helical (spiral) CT scanning is rapidly replacing conventional dynamic axial CT (sliceby-slice acquisition) in medical centers. Helical CT involves continuous rotation of the $\mathrm{x}$ ray tube and continuous exposure of the patient to the x-ray beam while translating through the gantry that allows rapid acquisition of volumetric data. This technique is faster, provides high-quality three-dimensional reconstruction without the step borders in the standard or conventional incremental axial scans. Retrospective reconstruction of the thinner sections is also possible with this acquisition. (71)

However, based on clinical background that axial CT scanning is diagnostically superior, decision was made to continue with using the conventional axial scanning technique. For this reason we worked initially to establish the CT EVD protocol. Specifications of the CT scan that follows the navigation system image protocol are: the gantry tilt, which specifically has to be zero-tilt, and the slice thickness, which should be $3 \mathrm{~mm}$ or less for the slice. The axial head CT scan slices in our hospital protocols were routinely set as 5 $\mathrm{mm}$ for the supratentorial region and $2.5 \mathrm{~mm}$ for the infratentorial region. In the CT EVD Protocol this was changed to $2.5 \mathrm{~mm}$ slices all through. A physicist at the department of radiology evaluated the radiation dose to determine the safety of this new protocol. The radiation dose was within the safe limit of the international radiation standard dose for head CT scan (Appendix E). In summary, for patients who were presenting to the emergency department with a TBI, head CT was done using the CT EVD Protocol. Having this protocol established for the study avoided an additional scan requirement when EVD placement is decided, and as such avoided delay while awaiting rescanning and extra radiation dose. 


\section{The neuronavigation system}

The StealthStation AxiEM electromagnetic navigation (Medtronic Navigation, Inc.) is a computer-aided, frameless image-guided stereotactic navigation system used for EVD placement. This is a software and hardware platform that can be used for real-time surgical navigation on patients' radiological images. The AxiEM system employs an electromagnetic localization system (EM localization system) to track instruments and anatomy simultaneously. The system functions by creating a magnetic field of a known intensity and then uses microsensors in key instruments to enable the StealthStation to know where the instrument is located relative to the patient's anatomy. Surgical components consist of a number of tools such tracer pointer, AxiEM stylet, non-invasive patient tracker and AxiEM mobile emitter. This system provides real-time, 2-dimentional (2-D) and 3-dimentional (3-D) visualization, and enables virtual tracking navigation (Appendix F).

For the steps of EVD placement procedure using the Medtronic AxiEM electromagnetic navigation, refer to the manual "Synergy Cranial AxiEM Pocket Guide" (Appendix G). (72) The navigation setup time is the time required to upload the images into the navigation system until creating the 3-D model and planning the target. The registration time is the time required to register the uploaded CT scan in the AxiEM system to the patient's head to be able to simulate real-time catheter placement. The procedure time is the time from the skin incision until skin closure. Prior to starting this project, an expert from the navigation team helped with the training to familiarize oneself about the system, practice doing the procedure independently on a phantom skull and overcome common problems that might be faced when running the system independently. The project was planned to work without the need for routine neuronavigation expert support. This was to assess the ease-to-use and feasibility of running this system.

\section{The EVD insertion procedure}

Classic freehand technique: The standard practice in our hospital was to place EVDs at the bedside in ICU. All these patients by the standard of severe TBI management were sedated and intubated. First the coagulation profile and platelets counts, and other possible coagulopathies were managed. After placing the head in neutral position and 
clipping the patient's hair, the physician carrying on the procedure put on a mask, a gown and sterile gloves. Meticulous prepping and draping were done in a fashion to expose the right frontal area. On a few occasions and according to clinical indications, the left frontal side was exposed instead. Local anesthetic agents (lidocaine with epinephrine) and intravenous antibiotics (cefazolin, or vancomycin if allergic to penicillin) were used in some cases. Classically, the scalp incision was made over the Kocher's point, which is about $10 \mathrm{~mm}$ anterior to the coronal suture in the mid-pupillary line and about $2.5 \mathrm{~cm}$ from midline, and then the periosteum was separated from the skull. The skull was drilled using a manual twist drill with care not to injure the dura or the underlying brain in a direction aiming towards the frontal horn of the ipsilateral ventricle. Irrigation with normal saline was used occasionally to remove bone dust and debris followed by sharp small dura opening. The stylet-loaded ventricular catheter (Medtronic, Barium Impregnated, $35 \mathrm{~cm}$, outer-diameter $2.8 \mathrm{~mm}$, inner-diameter $1.5 \mathrm{~mm}$ ) was inserted using external landmarks while maintaining an orthogonal trajectory with respect to the skull. This was done using the ipsilateral medial epicanthus plane coronally and the external auditory meatus plane sagitally, which corresponds to the desired target close to the foramen of Monro. Practically, the success or failure of freehand EVD placement is measured by the free flow of CSF from the distal end of the catheter. The distal end of the catheter was then subcutaneously tunneled and securely fixed to the skin to avoid inadvertent pullout, followed by skin wound closure and dressing covering. The distal end of the catheter is then attached to a draining and monitoring system (Medtronic, Duet External Drainage and Monitoring System).

Navigation technique: The same standard procedure and the same catheter used for the freehand group were used for the navigation group with few extra steps required for the neuronavigation setup. The CT images were uploaded form the hospital picture archiving and communication system (PACS) to the AxiEM electromagnetic neuronavigation system. The setup involved building the 3-D navigation model and selecting the target. The planning of the target point was a crucial part of the procedure, which was set close to the ipsilateral foramen of Monro. The registration took place when the patient arrived in ICU by using the surface registration over the face and forehead. After confirming the accuracy, the procedure advanced in steps similar to the ones followed in the classic EVD placement. The step of catheter insertion is solely done under the navigation guidance using the navigation stylet-loaded ventricular catheter towards the preselected 
target close to the foramen of Monro. Throughout the whole procedure the patient tracker should be kept strictly in position. The patient tracker is the main reference during the procedure and it is subjected to detach, hence, shall ensure it remains in position. After successful placement, again further steps continued the same as in the classic technique (Figure 1). The tools and the kits contents plus the process of using the system are explained in the manual provided by Medtronic Navigation Inc. "Synergy Cranial AxiEM Pocket Guide”. (72)

\section{Radiological evaluation}

A CT scan was done within 24 hours after the EVD insertion to verify the tip and rule out complications. In the time before the study period, this was also being done routinely at our institution. Using the hospital PACS system, post procedure CT scans of a mixed cohort of freehand group and navigation group were used each time for EVD tip accuracy evaluation. A neuroradiologist who was blinded to the patients' details and the technique used for EVD placement, reviewed the scans and classified the accuracy according to the Kakarla grades of EVD tip accuracy (Appendix C), in addition to reporting the post procedural radiological complications. Radiological complications included any new bleeding related to the EVD path. In most instances this was EVD track hemorrhage, and infrequently a subdural hematoma or subarachnoid hemorrhage. Pneumocephalus was not considered a complication. In pre-procedural scan, other measures were also performed and included the bifrontal ventricular width, hydrocephalus ratio, frontal ventricular size, caudate-septal line, bicaudate width and bicaudate index (Figure 2). The midline shift was also measured in pre-procedural scans (Figure 3), in addition to evaluating the CT scan structural appearance according to the Marshall grades (Appendix H). (73) Other radiological measures in post-insertion scans were evaluated such as the EVD catheter length measured from the outer table of the cranium at the burr hole to the tip (Figure 4), and the entry point evaluation plus the eloquent areas traversed by the catheter, including the corpus callosum.

\section{Clinical evaluation}

The number of passes was evaluated as recorded in the procedure note during the charts review. The details of first recorded ICP and number of passes are frequently 
documented in the procedure note. External ventricular drain stay was the number of days the catheter remained in-situ. The total hospital stay included the number of days from admission to discharge. The ICU stay indicated the number of days spent in the ICU initially and excluding other readmissions. The intubation period included the time from admission to extubation, and in case of having tracheostomy then this included the time until the patient was weaned off the ventilator. The patients who were eventually considered of guarded prognosis and subjected to extubation and comfort care measures of treatment were excluded from the evaluation of the ICU stay \& intubation period. The catheter revision included the replacement, and catheter pullout when deemed beyond the target in the post-procedure CT scan. Management of a misplaced EVD is based on clinical grounds; patients with functioning EVDs with the tip in an undesirable position were followed as long as the EVD was functioning. The malfunctioning EVD included the ones documented not to be draining or blocked with unsuccessful de-blocking trial, or eventually removed for the same reason as documented in the chart. The CSF infection was categorized into 3 groups: 1) no infection, 2) contamination when having positive CSF but the clinical picture did not suggest infection and no treatment was planned, and 3) infection when having positive CSF results and supported by clinical status requiring intravenous antibiotic therapy. The discharge status included 4 parameters: 1) home, 2) discharge to a rehabilitation center, 3) transfer to a long term care facility which included also the transfer to another hospital while awaiting placement at the long-term care facility, and 4) death when it occurred during the same admission.

\section{Statistical analysis}

All statistical analyses were performed using SPSS software (IBM, SPSS, Statistics version 20.0 for Mac OS; SPSS Inc., Chicago, IL, USA). We reported median or mean ( \pm standard deviation) for continuous variables, and count (\%) for categorical variables. While two groups were compared using Student's independent t-test for continuous variables, Chi-square test was used to compare groups for categorical variables. However, Fischer's exact test was used when more than one cell frequency was less than 5 in $2 \times 2$ contingency table. To compare more than two groups, we used ANOVA F-test and performed multiple comparisons using Bonferroni test. Multivariate logistic regression analysis was used to obtain the odds ratio (P-value) after adjusting for potential 
confounders. Note that in the multivariate analysis we considered only two categories for EVD tip accuracy rather than 3 categories. We merged grade 2 and grade 3 as a suboptimal group. This was to improve the accuracy of our results from the analysis, as only 3 cases belonged to grade 2 categories. The test was considered significant if $P$ value was less than 0.05 . 


\section{RESULTS}

As shown in Figure 5, over the 2 years study period, the reviewed cases of adult patients with severe TBI were 132 patients. Thirty were not included because they had no ICP monitors inserted. These were mainly patients who showed clinical improvement on admission and frequent evaluation was feasible with no signs supporting elevated ICP, and another group of patients who were deemed of guarded outcome prognosis or having evolving brain stem death, and their management was readjusted into palliative measures (Figure 6). There was one patient who died in the operating room. Out of the remaining 102 cases, there were 14 additional revision procedures giving a result of 116 total ICP monitors placed during the study period. The EVD cases that were inserted exclusively intraoperatively (49 cases) were not included; likewise, 10 cases of parenchymal ICP monitors were excluded (Figure 7). It is of interest to note that out of the 10 cases of parenchymal ICP monitors, in 3 cases the reason was failed freehand EVD insertion and in 1 case it was for anticipated EVD insertion difficulty. The eligible cases for evaluation in our study were 57 cases of EVD placement. Three further cases were excluded from the retrospective group; because of failed insertion and consequently no post procedural scan to be evaluated ( 2 cases), and in one case the patient died with no follow up CT scan done after the procedure. Ventricular catheter insertion failure is a valid outcome, however, these cases were excluded as the primary outcome necessitate reviewing the post placement scan. There were 2 cases that crossed over from the navigation group and were evaluated under the freehand group (Figure 8); one had CT scan done without using the CT EVD Protocol and one had failed the registration because of the late decision of EVD placement (5 hours post the CT scan) and that particular patient was having ongoing right periorbital and forehead swelling that made the registration not successful after many attempts. The final number of procedures recruited in the study was 54 case of EVD placement; 35 (64.8\%) were placed freehand and 19 (35.2\%) under navigation guidance (Figure 9).

\section{Baseline characteristics}

Baseline characteristics of the study population are provided in Table 2. A total of 50 patients underwent 54 EVD placement procedures using either the classic freehand 
technique (No. $=34)$ or the navigation guidance method (No. $=18)$. One case in the navigation group had the EVD revised after 4 days because of blockage with a clot, confirmed by examining the catheter after removal that failed to be dislodged with normal saline flushing of the EVD. At the first insertion, this was a difficult case because of the significant brain distortion. In both cases the insertion was successful from the first pass using the navigation (Figure 10).

The baseline demographics and severity of head injury were comparable between both groups. The mean age in years was $49.5 \pm 20.4$ for the freehand group and $48.2 \pm 21.6$ for navigation group. Males constitute most of the severe TBI population, and in our study $82.9 \%$ of the freehand group were males, and similarly $78.9 \%$ were males in the navigation group. Figure 11 shows the different mode of injuries, and fall was found to be the most common type of injuries for both groups. Collectively, the initial GCS of 8 or less, was about $60 \%$ in both groups with a median of 7 and 8 , respectively. The GCS score correlates with outcome $(22,23)$ and this refers to the best GCS after resuscitation. The resuscitation may also include emergent surgical intervention. As there was no consensus about the best timing to refer to the best GCS, we included in our study the best GCS in the emergency room in addition to recording the best GCS after 24 hours. Figure 12 shows the distribution of different GCS scores between both groups after 24 hours from admission. It is not uncommon to have the TBI associated with other systemic injury. About $20 \%$ of navigation group and $40 \%$ of freehand group were associated with multisystem injuries. Minimal injuries, such as rib fractures that were not associated with hemothorax or pneumothorax, vertebral transverse process fractures, other bony fractures that did not warrant surgical intervention and skin lacerations, were not included. Only 4 cases were subjected to emergency surgical intervention other than for TBI and all of these cases were in the freehand group $(\mathrm{P}-$ Value $=0.158)$. On the other hand, $47.4 \%$ had urgent surgery related to TBI in the navigation group, and this was $35.3 \%$ in the freehand group (P-Value $=0.389)$. Coagulopathy, including the use of antithrombotic or anticoagulant agents, or due to other causes like trauma, was reported in $32.4 \%$ of the freehand group and $21.1 \%$ of the navigation group $(\mathrm{P}-\mathrm{Value}=0.381)$. The first recorded ICP after ICP monitor placement is routinely recorded in the procedure notes as opening pressure in $\mathrm{mmHg}$. The mean pressure in the freehand group was $17.8 \pm$ 12.9 , and it was $14.7 \pm 8.8$ in the navigation group. The right side is routinely chosen for frontal ICP monitor placement because of the left side cerebral hemisphere representation 
of language and memory in most people. When it was otherwise indicated, the left side was used for insertion in $20.0 \%$ and $15.8 \%$ of the freehand and navigation groups, respectively. Magnetic resonance imaging (MRI) study is not done routinely in TBI population unless for certain indications. It is reasonable to investigate with MRI when depressed level of consciousness is not explained by elevated ICP measures or other clinical and biomedical measures. One of these indications is to evaluate for diffuse axonal injury (DAI), which is graded into 3 grades (Appendix H). (74) About $30 \%$ of our population had MRI study; out of these, $30 \%$ had no prove of DAI and this was evenly distributed between the two groups. The transfer from other hospitals that had no TBI specialist coverage was also evaluated in the baseline demographics, as this may be associated with higher mortality and worse outcome. (17) In regards to the transfer from other hospitals; there was no significant difference between the freehand group and the navigation group.

\section{Potential determinants of EVD placement accuracy}

There are many factors that can affect the EVD placement accuracy. Indeed, we selected to study the severe TBI population because it is an important determinant of catheter placement accuracy, and inserting ventricular catheter using freehand technique may be the only significant factor for misplacement as found in previous studies. $(2,61)$ Any factor can be studied separately as a potential, and in our study we included the factors that had been previously reported in literature and the ones that may have more clinical impact in this determination (Table 3). Large ventricular measurements may indicate a favorable case for catheter placement accuracy in which the target is physical larger. These factors were comparable for the freehand and the navigation groups, except for frontal horn size that was significantly smaller in the navigation group. Overall there is slight predilection towards having difficult EVD placement in the navigation group by having less favorable measures, with larger midline shift, and smaller Evan's index, bifrontal ventricular width, frontal horn size, caudate-septal line, bicaudate index, and less percentage of hydrocephalus in the navigation group. The frontal horn size was significantly smaller in the navigation group measuring $4.5 \pm 4.1 \mathrm{~mm}$ in comparison to $7.3 \pm 5.5 \mathrm{~mm}$ in the freehand group $\{(\mathrm{P}-$ Value $=0.039,95 \% \mathrm{CI}(-5.5,-0.1)\}$. The bifrontal ventricular width was also smaller in the navigation group measuring $32.6 \pm 6.7$ $\mathrm{mm}\{(\mathrm{P}-$ Value $=0.081,95 \% \mathrm{CI}(-7.6,0.5)\}$. There was no significant association 
between both groups in regards to Marshall's grades (Appendix I), and 48\% of the cases were similarly distributed in class II (diffuse injury II; cisterns are present with midline shift 0-5 mm and/or; lesion densities present, no high- or mixed-density lesion $>25 \mathrm{cc}$, may include bone fragments and foreign bodies). The level of training was significantly different between both groups $(\mathrm{P}-$ Value $=0.005)$ when evaluating association between different levels of training and the placement technique used. Figure 13, shows no significant association between the level of training and the accuracy of catheter placement when evaluating the entire cohort, however, there were more navigationguided placements done by residents in their fourth year of training.

Table 4 shows the results from the multivariate logistic regression model where we considered EVD tip accuracy as outcome (1= optimal, $0=$ suboptimal) and EVD placement technique using the navigation (Yes / No) as exposure. The effect of navigation use on outcome was adjusted by including the level of training, midline shift, hydrocephalus ratio, Marshall grade, bicaudate index, and ventricular size, as confounders in the model. The results show that the odds of getting optimal results was 20.8 [ $(=\exp$ (3.035)] times higher when navigation was used as compared to freehand insertion of EVD $(\mathrm{P}$-value $=0.010)$.

\section{Primary outcomes}

In our study hypothesis, the primary outcome was the accuracy of ventricular catheter tip placement when using the navigation to guide EVD placement in comparison to free hand insertion in severe TBI. The accuracy was evaluated using the Kakarla grades for the accuracy of the tip location. The neuroradiologist who was blinded to the technique used, evaluated this variable. In each session, a mixed cohort of scans of both groups was used in a random fashion. The results in Table 5 show significant $(\mathrm{P}-\mathrm{Value}=0.009)$ association between using the navigation guidance and having an optimal and adequate EVD placement (grade 1). Out of 19 cases in the navigation group, 18 (94.7\%) had grade 1 accuracy, one case (5.3\%) grade 2, and none grade 3. Figure 14 shows the case that had the EVD crossed to the contralateral horn and labeled under grade 2 accuracy. The degree of septum pellucidum shift in this case may have contributed to this result. The classic freehand group had optimal placement in $57.1 \%$ of the cases (grade 1) and suboptimal placement in eloquent tissue was in $37.1 \%$ (grade 3). Examples of grade 3 misplacement included the internal capsule, the basal cisterns, the thalamus and brainstem (Figure 15). 
The result of the freehand group placement was comparable with what has been previously reported in the literature (Table 1).

The mean number of passes for each procedure was the other primary outcome of our study. The record of passes was absent in $23 \%$ of the cases in the freehand group with no missing record in the navigation group. The number of passes was significantly lower in the navigation group with a mean of $1.16 \pm 0.38\{\mathrm{P}-$ Value $=0.018,95 \% \mathrm{CI}(-0.86,-$ $0.09)\}$. The freehand group mean was $1.63 \pm 0.88$, and for the entire cohort was $1.43 \pm$ 0.75 passes. Note that the two excluded cases from the retrospective cohort because of failed insertion were recorded to have 2 and 3 number of passes. Evaluating the number of passes in relation to the placement accuracy in Figure 16 revealed that the mean number of passes was statistically lower in grade 1 accuracy $(1.25 \pm 0.51)$, compared to grade $3(1.91 \pm 1.04),\{\mathrm{F}(2,45)=5.27, \mathrm{P}$-Value $=0.009\}$. Multiple passes was also associated with increasing risk of radiological complications (Figure 17). The mean number of passes in the procedures that had been reported to have hemorrhagic complications post insertion was $2.00 \pm 1.10\{\mathrm{P}-$ Value $=0.051,95 \% \mathrm{CI}(-0.005,1.491)\}$ as shown in Figure 18.

Practically, the success of freehand EVD placement is measured by the free flow of CSF from the distal end of the catheter during the insertion procedure. This may be misleading as in occasions where the catheter is in the desired place, but because the ICP is not high or in cases where some CSF was lost during the procedure, then the absence of CSF flow does not necessarily indicate placement failure. This was probably the case in the 2 cases where we had 2 passes each. The navigation confirmed that the tip was at the desired target, however, the tube was retrieved and reinserted during the same procedure and again targeted the same point. The follow up CT scan confirmed that the catheter is in the desired position, and the drain was working eventually in the following hour. In one of the two cases, there was a clot at the distal catheter; hence the catheter was replaced with a new one. In another following procedure, we avoided removing the catheter when it did not instantaneously drain CSF and it was eventually draining. In the other hand, having instantaneous CSF draining from the distal catheter at the time of insertion could be deceiving as in many cases the post-procedural CT scan confirmed catheter malposition. The Medtronic EDM Ventricular Catheters have multiple holes along the proximal 2.4 $\mathrm{cm}$ with 4 rows of 4 holes each, and the presence of one hole in the ventricular system might be sufficient to drain CSF at the time of insertion whereas the tip of the catheter is 
in an undesired place. Similarly, the presence of the catheter tip in potentially dangerous spaces containing CSF space like the basal cisterns can also be misleading.

We observed that the accuracy of the distal tip of the EVD catheter was not a strong enough determinant for the safety of the EVD placement. When thinking about the skull with the brain inside it as 3-D model, then at least an additional point is important to draw the line in this 3-D space to determine a safe path of insertion. Hence, we selected the other point as the entry point to access the skull for catheter placement and this was evaluated in the post-procedure CT scan. Safe path is the one that does not pass through the corpus callosum and other eloquent brain tissue including, but not limited to, the basal ganglia, the thalamus, the pre and post central gyri, the brain stem and the internal capsule. This point in our study highlights that the importance is not only at the tip, but also the entire path of the catheter. We divided the access point to the skull (the entry point) into 3 different grades; ideal being placed on the ipsilateral coronal suture or anterior to it and lateral to midline by more than $2.5 \mathrm{~cm}$, suboptimal when it is $1 \mathrm{~cm}$ to 2.5 $\mathrm{cm}$ lateral to the midline, and potentially dangerous when the entry point is posterior to the coronal sutures or within $1 \mathrm{~cm}$ from midline. Having the entry point grade as suboptimal or potentially dangerous was associated with more risk of having a path that is not safe for placement (Figure 19). The corpus callosum, the caudate nucleus and the thalamus were the most frequently traversed tissue when the pass was not safe. Using the navigation was associated with having a safe path for EVD placement in $84.2 \%$ compared to the freehand group with only $42.9 \%$ of the EVD placement procedures as having a safe path $(\mathrm{P}-$ Value $=0.03)$.

\section{Clinical outcomes}

The accuracy and number of passes are considered part of the EVD morbidities, however, their value in the over all clinical outcome may be difficult to estimate. Other clinical outcomes were also studied as secondary outcomes in our study (Table 6). There is a minor predilection towards having better clinical outcomes in the navigation group in comparison to the freehand group when evaluating the EVD stay prior to removal $(5.8 \pm$ 3.8 Vs. $6.5 \pm 4.2$ days), ICU admission duration (19.1 \pm 8.1 Vs. $20.0 \pm 8.8$ days), total hospital admission duration (mean: $28.7 \pm 23.2$ Vs. $45.1 \pm 57.0$ days, median: 21 Vs. 26 days), total days of intubation (13.2 \pm 7.3 Vs. $14.4 \pm 6.9$ days), risk of EVD malfunction (15.8\% Vs. $33.3 \%)$, risk of revisions (5.3\% Vs. $18.2 \%)$, risk of infection $(5.3 \%$ Vs. 
7.1\%), radiological complications (15.8\% Vs. 22.9\%) and death (47.4\% Vs. 54.3\%) as a final outcome. None of these secondary outcomes had reached statistical significance. It is important to note that the navigation expert personnel presence was not required for any of the cases when navigation was used. However, it was important to spend few hours prior to conducting the study to learn the basics of this navigation system when simulating its use as real-time in EVD placement. This was important to limit the cost of implementing this technology, and also avoiding unnecessary delays in patient's management. The navigation procedure involves additional time for the navigation setup and for the registration prior to implement it in the catheter guidance. The navigation guidance procedure time is divided into different parts. The navigation setting time includes uploading the scan from the PACS to the navigation system, checking the connectivity of the equipment, building the 3-D model and selecting the desired target. The navigation registration involves registration of the uploaded CT scan to the patient's head and confirming its accuracy before proceedings to the EVD insertion procedure. The actual procedure time is from skin incision to closure and this is the only part that is included in the freehand insertion technique. Figure 20 represents the time spent for each subdivision in the navigation group. The mean time for the navigation setting was $17.22 \pm$ 6.73 minutes and for the registration was $17.17 \pm 11.05$ minutes. These two parts were the additional time required for the navigation. However, when the decision to insert an EVD was made for a patient presenting to the emergency room, in almost all cases the investigator was present before the patients were transported to ICU. The navigation setup mean time of 17.22 minutes was almost always completed prior to the patients being physically in their beds in the ICU. The registration mean time of 17.17 minutes was the actual additional time, as it shall be done when the patient already is in his bed in ICU and deemed ready by the ICU team. The mean of the procedure time from skin opening to closure in our records for the navigation group was 28.67 minutes. We also kept records about the cannulation time, which is part of the procedure time, and we defined it as the time from skin opening until placing the catheter in the ventricle and the mean was 18.44 minutes. The importance of the cannulation time is that it is the actual time for decompression in case of acute hydrocephalus that needs to be drained immediately as the rest of the procedure time includes tunneling the catheter and securing it by applying few stitches holding it to the skin, and skin closure. It was not feasible to make comparison with the freehand group in regards to the procedure time, as this was 
not routinely recorded in the patient's chart. Another measure to note was the mean time from the $\mathrm{CT}$ scan to the procedure, which was $4.94 \pm 10.37$ hours after excluding an outlier in which case the scan that was used was done few days prior to the procedure. This was a patient who deteriorated clinically while managed in ICU without changes in his CT scan. In this case the immediate scan prior to the procedure was not done according to the CT EVD Protocol specifications. The follow up post-procedure CT scan mean time was $6.05 \pm 6.49$ hours after the insertion procedure.

In our study, for the EVD length, we measured the EVD catheter length to the outer table of the cranium and not to the inner table as in some other studies. The former is practically more relevant as markings of the length of the catheter can be visualized at the outer table of the skull during the procedure. Previously studied, the catheter length of 5.0 - $7.75 \mathrm{~cm}$ was associated with better accuracy. $(43,44,56)$ The mean of the EVD length in our study was $81.7 \pm 10.0 \mathrm{~mm}$ for the entire cohort, and the difference was not statistically different between the groups, $83.1 \pm 10.7 \mathrm{~mm}$ for the freehand group, and was $79.0 \pm 8.1 \mathrm{~mm}$ for the navigation group $\{\mathrm{P}-\mathrm{value}=0.123,95 \% \mathrm{CI}(-9.3,1.1)\}$. Figure 21 shows the association between the mean catheter length and the accuracy of EVD placement. The mean catheter length was $77.9 \pm 8.7 \mathrm{~mm}$ for grade 1 accuracy, $81.6 \pm 2.5$ $\mathrm{mm}$ for grade 2 , and $92.6 \pm 5.7 \mathrm{~mm}$ for grade 3 . The difference was significant between grade 1 and grade $3\{\mathrm{~F}(2,51)=16.7$, P-Value $<0.000\}$. It is important to note that the final location of the catheter tip may be different by few millimeters from the actual target planned in the navigation system and the main reason for this was that after placing the catheter, the later was still subjected to some minimal manipulation, like the subcutaneous tunneling. In our study, we did not evaluate this point, as we did not keep imaging records about our targets for each procedure to be able to compare it with the post-procedural catheter distal tip location. 


\section{DISCUSSION}

The ideal ventricular catheter placement is the one that: 1) has an optimal entry site to the skull, 2) has a safe path and distal tip ending at the targeted location close to the foramen of Monro, 3) from the first pass, 4) deemed functional with no post insertion complications, 5) is done in a short time and at an optimal time from when the decision is taken to insert a catheter, and 6) necessitates no additional cost (Figure 22). Freehand EVD insertion technique using superficial anatomical landmarks currently remains the method of choice due to its simplicity, and more importantly, its efficiency. This remains the most common method practiced by neurosurgeons and is currently the standard of care to access ventricular compartments. Ventriculostomy procedure is often placed in emergency settings, and because it is a potentially life-saving procedure, time is crucially important. The unreliability of freehand technique may be related to: intra-operative error, anatomical difference or suboptimal historical targeting landmarks. Using the ipsilateral medial canthus trajectory was found to be unreliable guide for directing an EVD whereas both the perpendicularity to skull and contralateral medial canthus trajectories were significantly more reliable methods for targeting the frontal horn of the ipsilateral lateral ventricle. (39) In another study using a virtual radiological analysis of 3-D data of skull and ventricular anatomy of randomly selected patients with normal ventricular anatomy, Rehman et al. reported $32 \%$ misplacement using virtual ventriculostomy trajectories at a perpendicular angle to the skull at the Kocher's point. (75) Additionally, scalp or forehead swelling associated with TBI and intracranial pathology including cerebral shift and edema can contribute to the challenge of the freehand insertion technique.

Ventricular catheter misplacement in eloquent brain tissue can result in significant morbidities that may have very serious consequences and necessitate further interventions. (45-49) The misplaced catheters may require revision, resulting in the lost benefit of therapeutic drainage, additional cost and time of repeat CT scan and procedures, and risk of additional brain injury. Each insertion pass results in more injuries to the already traumatized brain. An increase in the number of catheter passes is associated with a small but definite risk for complications, including hemorrhage, neurological injury, and infection. Post-procedure imaging studies, such as CT or MRI, will often show effects of trauma from the catheter's passes (Figure 23). There may or may not be associated clinically detectable findings from multiple catheter placement 
passes. Subtle neuropsychological effects may be present even when no clinically detectable effect is found. Hence, the number of passes should be minimized. The case presented in Figure 10 was an example of a challenging case for the use of freehand technique for EVD placement.

\section{Methods reported to improve EVD placement}

Different methods have evolved in an attempt to improve the accuracy of EVD placement. Ghajar Guide application, when introduced, was studied in 17 patients with good success rate from the first pass. Only 11 patients had confirmation of accurate placement using fluoroscopy. This is a rigid device and consists of four components: three equal length forming an equilateral triangular base and a central tube at the apex for passage of the catheter. This has been designed that, when placed over a burr hole, it guides a catheter in a path perpendicular to the plane tangent to the skull at the burr hole site. (41) The efficacy of the Ghajar Guide was revisited in a prospective study by O'Leary and associates comparing it with freehand technique and found that the mean number of passes was significantly lower in the Ghajar Guide group. The accuracy was evaluated according to the distance from the ipsilateral foramen of Monro rather than counting the actual misplacement. (42) Using the same simple principle of placing a device to the skull to guide a perpendicular catheter insertion, Yamada introduced a tripod to aid ventriculoperitoneal shunt insertion and found significantly better catheter tip accuracy when comparing the tripod method with freehand insertion. Again, the placement was not ideal in about $54 \%$ of the freehand group. (76) Garell et al. described a posterior burr hole localizer device (Localizer) that defines optimum burr hole location based on geometric relationships involving the ear and supraorbital rims. This may be useful in placement of occipital catheters such as ventriculoperitoneal shunts, but its use is limited in frontal EVD placement. (77) It should be noted that using the Ghajar Guide principle is useful only when the patient's anatomy has not been distorted and probably with large size ventricles. In trauma, it is also not uncommon to have scalp lacerations or contusions that may distort the perpendicular plane over the burr hole in addition to the intracranial anatomical distortions and brain shift. These limitations of the guide and the techniques with similar principles have resulted in the resistance to the use of this simple method to improve the accuracy of EVD placement. 
Image-guided methods of ventriculostomy catheter placement may minimize the number of passes and improve accuracy. A potential advantage of image-guided methods is onepass catheter insertion. This in itself may decrease procedural time. This is even more crucial when dealing with grossly distorted patient's anatomy when superficial anatomical landmarks are rendered unreliable. Ultrasound guidance and using CT scan and live fluoroscopic CT navigation guidance in radiology department to insert EVD were used in small numbers of case series with good placement accuracy and success from a single pass. (78-82) The time from CT to the initiation of procedure and the procedure time may be reduced as per Kortz and colleagues. (82) This may be true for patients who are already identified to require EVD placement at the scan time where the surgeon is readily available or when EVD placement is semi-elective. For patients who are already in ICU, extra transport time should be anticipated, adding to that the risk of radiation when it is done under $\mathrm{CT}$ guidance. This is in addition to the limited feasibility and cost, and the need for additional staff.

Endoscopy, stereotaxy and robotic have been described to improve accuracy in ventriculoperitoneal shunt insertion procedures. $(15,61,83-85)$ The feasibility of these methods is limited in urgent cases of severe TBI, and they require specific settings that may not be available outside the OR rooms. In addition, training simulation can be applied to EVD insertion training to improve the trainees' performance. $(86,87)$ Technologies can be used early in training like haptic-based simulator module that uses 3D platform for EVD placement and recreates the surface landmarks to guide the trajectory as well as providing the tactile feedback as the catheter passes through the parenchyma. Such simulators may be proven to be cost-effective for training neurosurgical residents in EVD placement and minimize risk of misplacement. $(88,89)$ Using smartphone application that can help setting the trajectory of a ventricular catheter-guiding tool was described as a simple method to improve the accuracy of ventricular catheter placement. (90)

\section{Novelty of our study and the limitations}

The navigation field continues to evolve and new tools are being developed in this field to make catheter placement easy to use, safe and accurate. (91) In a prospective study by Mahan et al. using the frameless electromagnetic navigation for EVD placement, accuracy was $94.1 \%$ with statistically significant improvement over historical data. (14) 
The novelty of our study is the targeted population of severe TBI who were repeatedly reported in literature to have high misplacement rates. This is the first study to prospectively test the use of this innovative technology to guide EVD placement in severe TBI. Establishing the CT EVD Protocol can be also counted as novel. This has minimized the need to repeat $\mathrm{CT}$ scan, minimizing the cost, the additional radiation exposure and the delay of treatment while transferring the patient to and from the radiology department for additional scans. The population in our study for both groups was from the same institute having the same conditions with same indications for catheter placement. The post procedure $\mathrm{CT}$ evaluation was subjected to blind evaluation and in each meeting there was always a mixture of cases from both cohorts. However, considering the application of the new scanning protocol, CT EVD Protocol, vigilant observation may verify the ones who had been subjected to this scanning protocol indicating that this case belongs to the prospective navigation group (Figure 24).

Randomized controlled study would have been ideal in this case, however, the cost, the time frame and precluding patients from having such an innovative and evolving technology that has been involved in all aspects of neurosurgery might raise an ethical dilemma. The image-guided technologies are considerably more expensive, requiring equipment that may be complex to use or bulky occupying large space, have limited capability for real-time guidance rendering them of questionable accuracy in case of ongoing anatomical distortion between the time of the scan and the time of procedure. Some fine details about the scan specifications for cases that require navigation guidance are important to pay attention to. An example is the case in Figure 25, in which you can notice that the patient was scanned while having the pulse oximetry connected to his forehead and occupying almost all the left side forehead. This made the registration difficult, as the forehead was the main area for surface registration, especially that the oximetry was removed after the scan. In the other hand, using fiducial markers can ensure a better accuracy, however, these were not found to be important and navigation was successful without then need for these markers. Furthermore, it would require that all patients in need for a CT for TBI screening to be equipped with fiducial markers, which is not a practical solution, given the time and the added cost.

The time required for setup time and registration of the CT scan to the patient plus making it available at every site when these procedures are being done in different locations are very important obstacles to adopt this technology in a routine daily practice, 
especially in cases when navigation technical support is required. There was an added time to set up the navigation system when using the navigation to aid placing the ventricular catheter but it was relatively short. The deficiency of the time record in the retrospective group made it difficult to reach a conclusion in regards to the time waste for the setup and registration and the over all time gain for the whole procedure. Having multiple passes when using the freehand method my actually involve extra time for the actual procedure time, and misplacement may involve an additional procedure for revision, and additional scan and cost. So, using the navigation may save time later as only one pass is required and the risk of malfunction and revision are also lower, and it certainly follows a learning curve in regards to time and experience as in any other technical methods in the medical field.

The advantage of accurate placement and less number of passes are evident in our study and that may lead to improved efficiency as well as safety. The modest improvement in revision and malfunction rates, and radiological complications, in such frequent procedure can lead to significant patient benefits. We postulate that some minor changes in the hardware setup and disposable kits can result in further improvement of this easyto-use portable system. A report including suggestions has been sent to the company at the midway through the study period with updates about this first prospective study of using navigation to guide ventricular catheter placement in trauma (Appendix J). 


\section{CONCLUSION}

Chasing perfection in EVD placement involves placement accuracy, and this is not currently attainable using the standard blind freehand technique, with its common risks of misplacement and multiple passes. Weather or not there is proof for increased morbidity from EVD inaccuracy; the demand is there to continue improving technologies that can serve this goal, and shall be sought when available. Despite adequate training and experience, ventricular catheter misplacement does occur frequently in severe TBI. Although many neurosurgeons believe that the current practice of freehand placement of ventricular catheter is good enough, the results of this study show that there is certainly much room for improvement. The easy-to-use and accurate electromagnetic navigation guidance is an innovative technique to guide ventricular catheter placement in severe TBI, and has the potential to reduce morbidities associated with this procedure. Using the electromagnetic navigation system to improve EVD placement accuracy is available, feasible, and accurate. Indication can be absolute when EVD placement is difficult as in TBI with brain swelling, compressed small ventricles and midline shift. 


\section{BIBLIOGRAPHY}

1. Srinivasan VM, O'Neill BR, Jho D, Whiting DM, Oh MY. The history of external ventricular drainage. J Neurosurg. 2014;120(1):228-36.

2. Kakarla UK, Chang SW, Theodore N, Spetzler RF, Kim LJ. Safety and Accuracy of Bedside External Ventricular Drain Placement. Neurosurgery. 2008;63:0NS162-ONS7. 3. Monro A. Observations on the structure and functions of the nervous system: Illustrated with tables. 1783.

4. Magendie F. Recherches anatomique et physiologique sur le liquide céphalorachidien ou cérebro-spinal. Paris, France. 1842.

5. Cushing H. Studies in Intracranial Physiology \& Surgery: The Third Circulation, the Hypophysics, the Gliomas: H. Milford, Oxford University Press; 1926.

6. Mokri BB. The Monro-Kellie hypothesis: applications in CSF volume depletion. Neurology. 2001;56(12):1746-8.

7. Fabrizi d'Acquapendente G. Opera chirurgica. in duas partes divisa ... Hieronymi Fabritii ab Aquapendente,... 2: Pars posterior : continens libros quinque chirurgiae. Francofurti: impensis Viduae et heredum Ionae Rosae [excudebat Nicolaus Hoffmannus]; 1619.

8. Missori P, Paolini S, Domenicucci M. The origin of the cannula for ventriculostomy in pediatric hydrocephalus. J Neurosurg Pediatrics. 2011;7(3):290-4. 9. Scott M. A Rubber Ventricular Cannula and Indwelling Catheter. J Neurosurg. $1953 ; 10(4): 438-9$.

10. Stangl AP. Continuous external CSF drainage--a perpetual problem in neurosurgery. Surg Neurol. 1998;50(1):77-82.

11. Arabi Y, Memish ZA, Balkhy HH, Francis C, Ferayan A, Al Shimemeri A, et al. Ventriculostomy-associated infections: incidence and risk factors. Am J Infect Control. 2005;33(3):137-43.

12. Holloway KL, Barnes T, Choi S, Bullock R, Marshall LF, Eisenberg HM, et al. Ventriculostomy infections: the effect of monitoring duration and catheter exchange in 584 patients. J Neurosurg. 1996;85(3):419-24.

13. Zabramski J. Efficacy of antimicrobial-impregnated external ventricular drain catheters: a prospective, randomized, controlled trial. J Neurosurg. 2003;98(4):725-30. 
14. Mahan M, Spetzler RF, Nakaji P. Electromagnetic stereotactic navigation for external ventricular drain placement in the intensive care unit. Journal of Clinical Neuroscience. 2013;20(12):1718-22.

15. Flannery AM, Duhaime AC, Tamber MS, Kemp J, Pediatric Hydrocephalus Systematic R, Evidence-Based Guidelines Task F. Pediatric hydrocephalus: systematic literature review and evidence-based guidelines. Part 3: Endoscopic computer-assisted electromagnetic navigation and ultrasonography as technical adjuvants for shunt placement. Journal of neurosurgery Pediatrics. 2014;14 Suppl 1:24-9.

16. Brain Trauma Foundation, American Association of Neurological Surgeons (AANS), Congress of Neurological Surgeons (CNS), AANS/CNS Joint Section on Neurotrauma and Critical Care, Bratton SL, et al. Guidelines for the Management of Severe Traumatic Brain Injury, VI. Indications for Intracranial Pressure Monitoring. J Neurotrauma. 2007;24, Suppl 1:S37-S44.

17. Ghajar J. Traumatic brain injury. The Lancet. 2000;356(9233):923-9.

18. Maas AI, Stocchetti N, Bullock R. Moderate and severe traumatic brain injury in adults. Lancet neurol. 2008;7(8):728-41.

19. Sosin DM, Sniezek JE, Waxweiler RJ. Trends in death associated with traumatic brain injury, 1979 through 1992: Success and failure. JAMA. 1995;273(22):1778-80. 20. Centers for Disease Control and Prevention. Injury Prevention \& Control: Traumatic Brain Injury [updated February 24, 2014; cited 2015 June 26]. Available from: http://www.cdc.gov/traumaticbraininjury/data/dist_death.html.

21. Yan SC, Smith TR, Bi WL, Brewster R, Gormley WB, Dunn IF, et al. The Assassination of Abraham Lincoln and the Evolution of Neuro-Trauma Care: Would the 16th President Have Survived in the Modern Era? World Neurosurg. 2015(0).

22. Teasdale GG. Assessment of coma and impaired consciousness. A practical scale. Lancet, The. 1974;2(7872):81-4.

23. Matis Georgios G. The Glasgow Coma Scale--a brief review. Past, present, future. Acta Neurol Belg. 2008;108(3):75-89.

24. Marshall LF, Smith RW, Shapiro HM. The outcome with aggressive treatment in severe head injuries. J Neurosurg. 1979;50(1):20-5.

25. Badri S, Chen J, Barber J, Temkin NR, Dikmen SS, Chesnut RM, et al. Mortality and long-term functional outcome associated with intracranial pressure after traumatic brain injury. Intensive Care Med. 2012;38(11):1800-9. 
26. Farahvar A, Gerber LM, Chiu YL, Carney N, Hartl R, Ghajar J. Increased mortality in patients with severe traumatic brain injury treated without intracranial pressure monitoring. J Neurosurg. 2012;117(4):729-34.

27. Talving P, Karamanos E, Teixeira PG, Skiada D, Lam L, Belzberg H, et al. Intracranial pressure monitoring in severe head injury: compliance with Brain Trauma Foundation guidelines and effect on outcomes: a prospective study. J Neurosurg. 2013;119(5):1248-54.

28. Lane PL, Skoretz TG, Doig G, Girotti MJ. Intracranial pressure monitoring and outcomes after traumatic brain injury. Can J Surg. 2000;43(6):442-8.

29. Stein DM, Hu PF, Brenner M, Sheth KN, Liu KH, Xiong W, et al. Brief episodes of intracranial hypertension and cerebral hypoperfusion are associated with poor functional outcome after severe traumatic brain injury. J Trauma. 2011;71(2):364-73; discussion 73-4.

30. Cremer OL, van Dijk GW, van Wensen E, Brekelmans GJF, Moons KGM, Leenen $\mathrm{LPH}$, et al. Effect of intracranial pressure monitoring and targeted intensive care on functional outcome after severe head injury*. Critical Care Medicine. 2005;33(10):220713.

31. Shafi S, Diaz-Arrastia R, Madden C, Gentilello L. Intracranial pressure monitoring in brain-injured patients is associated with worsening of survival. J Trauma. 2008;64(2):335-40.

32. Tang A, Pandit V, Fennell V, Jones T, Joseph B, O'Keeffe T, et al. Intracranial pressure monitor in patients with traumatic brain injury. Journal of Surgical Research. 2015;194(2):565-70.

33. Yuan Q, Wu X, Sun Y, Yu J, Li Z, Du Z, et al. Impact of intracranial pressure monitoring on mortality in patients with traumatic brain injury: a systematic review and meta-analysis. J Neurosurg. 2015;122(3):574-87.

34. Chesnut RM, Temkin N, Carney N, Dikmen S, Rondina C, Videtta W, et al. A trial of intracranial-pressure monitoring in traumatic brain injury. N Engl J Med. 2012;367(26):2471-81.

35. Brain Trauma Foundation, American Association of Neurological Surgeons (AANS), Congress of Neurological Surgeons (CNS), AANS/CNS Joint Section on Neurotrauma and Critical Care, Bratton SL, et al. Guidelines for the management of 
severe traumatic brain injury. VII. Intracranial Pressure Monitoring Technology. J Neurotrauma. 2007;24, Suppl 1:S45-S54.

36. Anderson RC, Kan P, Klimo P, Brockmeyer DL, Walker ML, Kestle JR. Complications of intracranial pressure monitoring in children with head trauma. J Neurosurg. 2004;101(1 Suppl):53-8.

37. Liu H, Wang W, Cheng F, Yuan Q, Yang J, Hu J, et al. External Ventricular Drains versus Intraparenchymal Intracranial Pressure Monitors in Traumatic Brain Injury: A Prospective Observational Study. World Neurosurg. 2015;83(5):794-800.

38. McGraw CP. Continuous intracranial pressure monitoring: review of techniques and presentation of method. Surg Neurol. 1976(3):149-55.

39. Muirhead WR, Basu S. Trajectories for frontal external ventricular drain placement: virtual cannulation of adults with acute hydrocephalus. Br J Neurosurg. 2012;26(5):710-6.

40. Friedman WAW. Percutaneous tunnel ventriculostomy. Summary of 100 procedures. J Neurosurg. 1980;53(5):662-5.

41. Ghajar J. A guide for ventricular catheter placement. J Neurosurg. 1985;63(6):985-6.

42. O'Leary ST, Kole MK, Hoover DA, Hysell SE, Thomas A, Shaffrey CI. Efficacy of the Ghajar Guide revisited: a prospective study. J Neurosurg. 2000;92(5):801-3.

43. Lind CR, Correia JA, Law AJ, Kejriwal R. A survey of surgical techniques for catheterising the cerebral lateral ventricles. Journal of Clinical Neuroscience. 2008;15(8):886-90.

44. Lind CR, Tsai AM, Law AJ, Lau H, Muthiah K. Ventricular catheter trajectories from traditional shunt approaches: a morphometric study in adults with hydrocephalus. J Neurosurg. 2008;108(5):930-3.

45. Chai FY, Farizal F, Jegan T. Coma due to malplaced external ventricular drain. Turk. 2013;23(4):561-3.

46. Grandhi R, Zwagerman NT, Lee P, Jovin T, Okonkwo DO. Iatrogenic pseudoaneurysm of the middle meningeal artery after external ventricular drain placement. J Neuroimaging. 2015;25(1):140-1.

47. Kosty J, Pukenas B, Smith M, Storm PB, Zager E, Stiefel M, et al. Iatrogenic vascular complications associated with external ventricular drain placement: a report of 
8 cases and review of the literature. Neurosurgery. 2013;72(2 Suppl Operative):ons20813; discussion ons13.

48. Schuette AJ, Blackburn SL, Barrow DL, Cawley CM. Pial arteriovenous fistula resulting from ventriculostomy. World Neurosurg. 2012;77(5-6):785.e1-2.

49. Rosenbaum BP, Wheeler AM, Krishnaney AA. External ventricular drain placement causing upgaze palsy: case report. Clin Neurol Neurosurg. 2013;115(8):1514-6.

50. Phillips SB, Delly F, Nelson C, Krishnamurthy S. Bedside External Ventricular Drain Placement: Can Multiple Passes Be Predicted on the Computed Tomography Scan Before the Procedure? World Neurosurg. 2014;82(5):739-44.

51. Huyette DR, Turnbow BJ, Kaufman C, Vaslow DF, Whiting BB, Oh MY. Accuracy of the freehand pass technique for ventriculostomy catheter placement: retrospective assessment using computed tomography scans. J Neurosurg. 2008;108(1):88-91. 52. Evans WA, Jr. An encephalographic ratio for estimating the size of the cerebral ventricles: Further experience with serial observations. American Journal of Diseases of Children. 1942;64(5):820-30.

53. Toma AK, Camp S, Watkins LD, Grieve J, Kitchen ND. External ventricular drain insertion accuracy: is there a need for change in practice? Neurosurgery. 2009;65(6):1197-200; discussion 200-1.

54. Lee JH, Park CW, Lee U, Kim YB, Yoo CJ, Kim EY, et al. Accuracy of the free hand placement of an external ventricular drain (EVD). Korean Journal of Cerebrovascular Surgery. 2010;12(2):82-6.

55. Hsieh CT, Chen GJ, Ma HI, Chang CF, Cheng CM, Su YH, et al. The misplacement of external ventricular drain by freehand method in emergent neurosurgery. Acta Neurol Belg. 2011;111(1):22-8.

56. Abdoh MG, Bekaert O, Hodel J, Diarra SM, Le Guerinel C, Nseir R, et al. Accuracy of external ventricular drainage catheter placement. Acta Neurochir (Wien). 2012;154(1):153-9.

57. Park YG, Woo HJ, Kim E, Park J. Accuracy and Safety of Bedside External Ventricular Drain Placement at Two Different Cranial Sites : Kocher's Point versus Forehead. Journal of Korean Neurosurgical Society. 2011;50(4):317-21.

58. Foreman PM, Hendrix P, Griessenauer CJ, Schmalz PGR, Harrigan MR. External ventricular drain placement in the intensive care unit versus operating room: 
Evaluation of complications and accuracy. Clinical Neurology and Neurosurgery. 2015;128:94-100.

59. Saladino A, White JB, Wijdicks EF, Lanzino G. Malplacement of ventricular catheters by neurosurgeons: a single institution experience. Neurocrit Care. $2009 ; 10(2): 248-52$.

60. Bogdahn UU. Continuous-pressure controlled, external ventricular drainage for treatment of acute hydrocephalus--evaluation of risk factors. Neurosurgery. 1992;31(5):898-903.

61. Wilson TJ, Stetler WR, Jr., Al-Holou WN, Sullivan SE. Comparison of the accuracy of ventricular catheter placement using freehand placement, ultrasonic guidance, and stereotactic neuronavigation. J Neurosurg. 2013;119(1):66-70.

62. Ngo QN, Ranger A, Singh RN, Kornecki A, Seabrook JA, Fraser DD. External ventricular drains in pediatric patients. Pediatr Crit Care Med. 2009;10(3):346-51. 63. Siesjo P. The enigma of external ventricular drain placement. World Neurosurg. 2014;82(5):597-8.

64. García-Molina AA. [Phineas Gage and the enigma of the prefrontal cortex]. Neurología (Barcelona, Spain). 2012;27(6):370-5.

65. Guidotti TL. Phineas Gage and his frontal lobe-the "American Crowbar Case". Arch Environ Occup Health. 2012;67(4):249-50.

66. Stroszczynski C. Minimally Invasive Tumor Therapies. P.M. Schlag, H.-J. Senn, editors. Verlag Berlin Heidelberg: Springer; 2006.

67. Hayhurst C, Byrne P, Eldridge PR, Mallucci CL. Application of electromagnetic technology to neuronavigation: a revolution in image-guided neurosurgery. J Neurosurg. 2009;111(6):1179-84.

68. Kandasamy J, Hayhurst C, Clark S, Jenkinson MD, Byrne P, Karabatsou K, et al. Electromagnetic stereotactic ventriculoperitoneal csf shunting for idiopathic intracranial hypertension: a successful step forward? World Neurosurg. 2011;75(1):155-60; discussion 32-3.

69. Koivukangas T, Katisko JP, Koivukangas JP. Technical accuracy of optical and the electromagnetic tracking systems. Springerplus. 2013;2(1):90.

70. Franz Alfred MA. Electromagnetic tracking in medicine--a review of technology, validation, and applications. IEEE Transactions on Medical Imaging. 2014;33(8):170225. 
71. Wippold FJ. Head and neck imaging: the role of CT and MRI. J Magn Reson Imaging. 2007;25(3):453-65.

72. Medtronic Navigation Inc. Synergy Cranial AxiEM Pocket Guide. Louisville: Medtronic Navigation; 2008, Revision 4, 01/2009. p. 10-54.

73. Marshall LF, Marshall SB, Klauber MR, Clark MB, Eisenberg HM, Jane JA, et al. A new classification of head injury based on computerized tomography. Special Supplements. 1991;75(1s):S14-S20.

74. Adams JH. Diffuse axonal injury in head injury: definition, diagnosis and grading. Histopathology. 1989;15(1):49.

75. Rehman T, Rehman A, Ali R, Rehman A, Bashir H, Ahmed Bhimani S, et al. A radiographic analysis of ventricular trajectories. World Neurosurg. 2013;80(1-2):173-8. 76. Yamada SM, Yamada S, Goto Y, Nakaguchi H, Murakami M, Hoya K, et al. A simple and consistent technique for ventricular catheter insertion using a tripod. Clin Neurol Neurosurg. 2012;114(6):622-6.

77. Garell PC, Mirsky R, Noh MD, Loftus CM, Hitchon PW, Grady MS, et al. Posterior ventricular catheter burr-hole localizer. J Neurosurg. 1998;89(1):157-60.

78. Fiorella D, Peeling L, Denice CM, Sarmiento M, Woo HH. Integrated flat detector CT and live fluoroscopic-guided external ventricular drain placement within the neuroangiography suite. Journal of neurointerventional surgery. 2014;6(6):457-60. 79. Phillips SB, Gates M, Krishnamurthy S. Strategic placement of bedside ventriculostomies using ultrasound image guidance: report of three cases. Neurocrit Care. 2012;17(2):255-9.

80. Cooke DL, Levitt M, Kim LJ, Hallam DK, Ghodke B. Transcranial access using fluoroscopic flat panel detector CT navigation. AJNR Am J Neuroradiol. 2011;32(4):E6970.

81. Ruchholtz S, Waydhas C, Muller A, Lewan UM, Nast-Kolb D, Euler E, et al. Percutaneous computed tomographic-controlled ventriculostomy in severe traumatic brain injury. J Trauma. 1998;45(3):505-11.

82. Krotz M, Linsenmaier U, Kanz KG, Pfeifer KJ, Mutschler W, Reiser M. Evaluation of minimally invasive percutaneous CT-controlled ventriculostomy in patients with severe head trauma. Eur Radiol. 2004;14(2):227-33.

83. Lollis SS, Roberts DW. Robotic placement of a CNS ventricular reservoir for administration of chemotherapy. Br J Neurosurg. 2009;23(5):516-20. 
84. Crowley RW, Dumont AS, Asthagiri AR, Torner JC, Medel R, Jane JA, Jr., et al. Intraoperative ultrasound guidance for the placement of permanent ventricular cerebrospinal fluid shunt catheters: a single-center historical cohort study. World Neurosurg. 2014;81(2):397-403.

85. Gautschi OP, Smoll NR, Kotowski M, Schatlo B, Tosic M, Stimec B, et al. Nonassisted versus neuro-navigated and XperCT-guided external ventricular catheter placement: a comparative cadaver study. Acta Neurochir (Wien). 2014;156(4):777-85; discussion 85.

86. Banerjee PP, Luciano CJ, Lemole GM, Jr., Charbel FT, Oh MY. Accuracy of ventriculostomy catheter placement using a head- and hand-tracked high-resolution virtual reality simulator with haptic feedback. J Neurosurg. 2007;107(3):515-21. 87. Krombach G, Ganser A, Fricke C, Rohde V, Reinges M, Gilsbach J, et al. Virtual placement of frontal ventricular catheters using frameless neuronavigation: an "unbloody training" for young neurosurgeons. Minim Invasive Neurosurg. 2000;43(4):171-5.

88. Lemole GMJ, Banerjee PP, Luciano C, Neckrysh S, Charbel FT. Virtual reality in neurosurgical education: part-task ventriculostomy simulation with dynamic visual and haptic feedback. Neurosurgery. 2007;61(1):142-8; discussion 8-9.

89. Tai BL, Rooney D, Stephenson F, Liao PS, Sagher O, Shih AJ, et al. Development of a 3D-printed external ventricular drain placement simulator: technical note. J Neurosurg. 2015;123(4):1070-6.

90. Thomale UW, Knitter T, Schaumann A, Ahmadi SA, Ziegler P, Schulz M, et al. Smartphone-assisted guide for the placement of ventricular catheters. Childs Nerv Syst. 2013;29(1):131-9.

91. Stieglitz LH, Giordano M, Samii M, Luedemann WO. A new tool for frameless stereotactic placement of ventricular catheters. Neurosurgery. 2010;67(3 Suppl Operative):ons131-5; discussion ons5. 
TABLES 


\section{Table 1: Summary of external ventricular drain (EVD) placement accuracy in the published literatures}

Table: Summary of EVD placement accuracy in the published literatures

\begin{tabular}{|c|c|c|c|c|c|c|c|c|c|c|}
\hline \multirow[t]{2}{*}{ Article (publishing year) } & \multirow[t]{2}{*}{ Technique } & \multirow{2}{*}{$\begin{array}{l}\text { TBI } \\
\text { Indication }\end{array}$} & \multirow{2}{*}{$\begin{array}{l}\text { No. of } \\
\text { procedures }\end{array}$} & \multicolumn{2}{|c|}{ Kakarla Grade } & \multirow[b]{2}{*}{ Grade 3} & \multicolumn{2}{|c|}{ Multiple passes } & \multirow{2}{*}{$\begin{array}{l}\text { Mean distance } \\
\text { from FOM }(\mathrm{mm})\end{array}$} & \multirow{2}{*}{$\begin{array}{l}\text { Intracranial } \\
\text { length (mm) }\end{array}$} \\
\hline & & & & Grade $1^{\pi}$ & Grade 2 & & $\%$ & Mean & & \\
\hline Ghajar $(1985)^{\S}(41)$ & Ghajar Guide & Non TBI & $10 / 17$ & $100.0 \%$ & - & - & $0.0 \%$ & 1.00 & & \\
\hline Bogdahn et al. (1992) (60) & Free-hand & Non TBI ${ }^{\text {กศा }}$ & 100 & & & $11 \%^{\star}$ & $4.0 \%$ & & & \\
\hline angl et al. (1998) (10) & Free-hand ${ }^{*}$ & TBI (32\%) & 212 & & & $7.0 \%$ & $9.0 \%$ & 1.10 & & \\
\hline \multirow[t]{2}{*}{ O'Leary et al. (2000) (42) } & Ghajar Guide & \multirow[t]{2}{*}{ TBI (10.2\%) } & $25 / 49$ & & & & & 1.10 & $3.7 \pm 5.7$ & \\
\hline & Free-hand & & $24 / 49$ & & & & & 1.50 & $9.7 \pm 6.3$ & \\
\hline Anderdson et al. (2004)* (36) & Free-hand & TBI & 68 & & & $8.8 \%$ & & & & \\
\hline Huyette et al. (2008) (51) & Free-hand & TBI (51.5\%) & 98 & $56.1-64.3 \%$ & $13.2 \%$ & $22.4 \%$ & & 2.17 & $16 \pm 9.6$ & $87.4 \pm 14.0$ \\
\hline \multirow[t]{3}{*}{ Kakarla et al. (2008) (2) } & \multirow[t]{3}{*}{ Free-hand } & Over all & 346 & $76.9 \%$ & $9.8 \%$ & $13.3 \%$ & & & & \\
\hline & & TBI (18.9\%) & 64 & $56.3 \%$ & $14.0 \%$ & $29.7 \%$ & & & & \\
\hline & & SAH (44.2\%) & 153 & $85.6 \%$ & $6.5 \%$ & $7.8 \%$ & & & & \\
\hline Saladino et al. (2009) (59) & Free-hand $^{\delta}$ & TBI (5.1\%) & 212 & & & $12.3 \%$ & & & & \\
\hline Ngo et al. (2009)* (62) & Free-hand & TBI (36\%) & 96 & & & $6.3 \%$ & & & & \\
\hline Toma et al. (2009) (53) & Free-hand & Non TBI & $183 / 234$ & $39.9-59.0 \%$ & $20.7 \%$ & $20.2 \%$ & & & & $66 \pm 11.8$ \\
\hline Lee et al. (2010) (54) & Free-hand & Non TBI & 113 & $42.5-62.0 \%$ & $26.5 \%$ & $11.5 \%$ & & & & $57 \pm 8.4$ \\
\hline Park et al. (2011) (57) & Free-hand $^{\Phi}$ & TBI (1.6\%) & 250 & $88.4 \%$ & $4.8 \%$ & $6.8 \%$ & & & & \\
\hline Hsieh et al. (2011) (55) & Free-hand & TBI (31.7\%) & 129 & $60.5-70.5 \%$ & $16.3 \%$ & $13.4 \%$ & & & & \\
\hline Abdoh et al. (2012) (56) & Free-hand & Non TBI & 66 & & & & & & & 60 \\
\hline Mahan et al. (2013) (14) & Navigation & Non TBI & 35 & $94.1 \%$ & $5.9 \%$ & - & $20.0 \%$ & 1.60 & & \\
\hline Phillips et al. (2014) (50) & Free-hand & Non TBI & 47 & & & & $28.0 \%$ & 1.85 & & \\
\hline Foreman et al. (2015) (58) & Free-hand & TBI (6.7\%) & 138 & $63.8 \%$ & $31.2 \%$ & $5.1 \%$ & & & & \\
\hline
\end{tabular}

External Ventricular Drain (EVD); Traumatic Brain Injury (TBI); Foramen of Monro (FOM); Subarachanoid hemorrhage (SAH).

ๆ The range in grade 1 is mainly because of the addition of the tips located in 3rd ventricle when it is not clear if they pass through FOM or not

$\S$ Confirmation was done only in $11 / 17$ cases

ๆी The indication was acute hydrocephalus

$\star$ It is not clear how misplacement was diagnosed

* Using spinal needle instead of polymer plastic catheters

\$ Pediatric population

$\delta$ Included EVD (79.7\%), \& ventriculoperitoneal \& ventriculoatrial shunts (20.3\%) placement

$\Phi$ This is sum of 2 groups with 2 different frontal acces; Kocher's point and forehead

() Reference 
Table 2: Baseline demographics of patients

Table: Baseline demographics of patients undergoing EVD placement

\begin{tabular}{|c|c|c|c|c|c|}
\hline \multirow{2}{*}{\multicolumn{2}{|c|}{ Baseline demographics }} & & \multicolumn{2}{|c|}{ EVD Placement Technique } & \multirow[b]{2}{*}{ P Value } \\
\hline & & Entire Cohort & Freehand-Classic & Navigation-Guided & \\
\hline \multicolumn{2}{|c|}{ No. of procedures } & 54 & $35(64.8 \%)$ & $19(35.2 \%)$ & \\
\hline \multicolumn{2}{|c|}{ No. of patients } & 50 & 34 & 18 & \\
\hline \multicolumn{2}{|c|}{ Mean age in yrs } & $49.0 \pm 20.6$ & $49.5 \pm 20.4$ & $48.2 \pm 21.6$ & $0.831^{\S}$ \\
\hline \multirow[t]{2}{*}{ Sex } & Males & $44(81.5 \%)$ & $29(82.9 \%)$ & $15(78.9 \%)$ & \multirow[t]{2}{*}{0.724} \\
\hline & Females & $10(18.5 \%)$ & $6(17.1 \%)$ & $4(21.1 \%)$ & \\
\hline \multirow[t]{3}{*}{ Initial GCS } & $>8$ & $21(41.2 \%)$ & $13(40.6 \%)$ & $8(42.1 \%)$ & \multirow[t]{5}{*}{0.439} \\
\hline & $5-8$ & $23(45.1 \%)$ & $16(50.0 \%)$ & $7(36.8 \%)$ & \\
\hline & $<5$ & $7(13.7 \%)$ & $3(9.4 \%)$ & $4(21.1 \%)$ & \\
\hline \multicolumn{2}{|c|}{ Median initial GCS } & 7 & 7 & 8 & \\
\hline \multicolumn{2}{|c|}{ Median GCS after 24 hours } & 7 & 7 & 7 & \\
\hline \multicolumn{2}{|c|}{ Transfer from another hospital } & $25(46.3 \%)$ & $15(42.9 \%)$ & $10(52.6 \%)$ & 0.492 \\
\hline \multicolumn{2}{|c|}{ Isolated head injury } & $36(66.7 \%)$ & $21(60.0 \%)$ & $15(78.9 \%)$ & 0.158 \\
\hline \multicolumn{2}{|c|}{ Urgent TBI surgery } & $21(39.6 \%)$ & $12(35.3 \%)$ & $9(47.4 \%)$ & 0.389 \\
\hline \multicolumn{2}{|c|}{ Emergency surgery other than TBI } & $4(7.5 \%)$ & $4(11.8 \%)$ & $0(0.0 \%)$ & 0.158 \\
\hline \multicolumn{2}{|l|}{ Coagulopathy } & $15(28.3 \%)$ & $11(32.4 \%)$ & $4(21.1 \%)$ & 0.381 \\
\hline \multicolumn{2}{|c|}{ ICP opening pressure } & $16.65 \pm 11.5$ & $17.8 \pm 12.9$ & $14.7 \pm 8.8$ & $0.309^{9}$ \\
\hline \multirow[t]{2}{*}{ Insertion side } & Right Frontal & $44(81.5 \%)$ & $28(80.0 \%)$ & $16(84.2 \%)$ & \multirow[t]{2}{*}{0.704} \\
\hline & Left Frontal & $10(18.5 \%)$ & $7(20.0 \%)$ & $3(15.8 \%)$ & \\
\hline \multirow[t]{4}{*}{ DAl in MRI } & No DAI & $5(31.2 \%)$ & $3(30.0 \%)$ & $2(33.3 \%)$ & \multirow[t]{4}{*}{0.574} \\
\hline & DAl grade I & $1(6.2 \%)$ & $0(0.0 \%)$ & $1(16.7 \%)$ & \\
\hline & DAl grade II & $4(25.0 \%)$ & $3(30.0 \%)$ & $1(16.7 \%)$ & \\
\hline & DAl grade III & $6(37.5 \%)$ & $4(40.0 \%)$ & $2(33.3 \%)$ & \\
\hline
\end{tabular}

External ventricular drain (EVD); Glasgow come scale (GCS); Traumatic brain injury (TBI); Intracranial pressure (ICP); Diffuse axonal injury (DAI); Magnetic resonance image (MRI) $\S 95 \% \mathrm{Cl}(-13.5,11.0)$

ๆ $95 \% \mathrm{Cl}(-9.2,3.0)$ 
Table 3: Potential determinants of external ventricular drain (EVD) placement

Table: Potential deteminants of EVD placement accuracy

EVD Placement Technique

\begin{tabular}{|c|c|c|c|c|c|c|}
\hline \multicolumn{2}{|l|}{ Factors } & Entire Cohort & Freehand-Classic & Navigation-Guided & P Value & $95 \% \mathrm{Cl}$ \\
\hline \multicolumn{2}{|c|}{ Midline Shift (mm) } & $2.2 \pm 3.0$ & $2.1 \pm 3.3$ & $2.3 \pm 2.6$ & 0.871 & $(-1.5,1.8)$ \\
\hline \multicolumn{2}{|c|}{ Hydrocephalus ratio $^{\S}$} & $0.27 \pm 0.06$ & $0.28 \pm 0.06$ & $0.25 \pm 0.06$ & 0.102 & $(-0.06,0.01)$ \\
\hline \multicolumn{2}{|c|}{ Bifrontal Ventricular Width (mm) } & $34.9 \pm 7.3$ & $36.1 \pm 7.5$ & $32.6 \pm 6.7$ & 0.081 & $(-7.6,0.5)$ \\
\hline \multicolumn{2}{|c|}{ Frontal Horn Size (mm) } & $6.3 \pm 5.2$ & $7.3 \pm 5.5$ & $4.5 \pm 4.1$ & 0.039 & $(-5.5,-0.1)$ \\
\hline \multicolumn{2}{|c|}{ Caudate-Septal Line $(\mathrm{mm})$} & $8.6 \pm 4.3$ & $9.3 \pm 4.4$ & $7.4 \pm 4.0$ & 0.118 & $(-4.3,0.5)$ \\
\hline \multicolumn{2}{|c|}{ Bicaudate Width $(\mathrm{mm})$} & $19.1 \pm 8.4$ & $20.0 \pm 9.0$ & $17.5 \pm 7.1$ & 0.264 & $(-7.0,2.0)$ \\
\hline \multicolumn{2}{|l|}{ Bicaudate Index } & $0.17 \pm 0.12$ & $0.19 \pm 0.15$ & $0.14 \pm 0.06$ & 0.131 & $(-0.10,0.01)$ \\
\hline \multicolumn{2}{|c|}{ EVD Length $(\mathrm{mm})$} & $81.7 \pm 10.0$ & $83.1 \pm 10.7$ & $79.0 \pm 8.1$ & 0.123 & $(-9.3,1.1)$ \\
\hline \multirow[t]{2}{*}{ Brain Edema } & Yes & $43(79.6 \%)$ & $27(77.1 \%)$ & $16(84.2 \%)$ & \multirow[t]{2}{*}{0.538} & \\
\hline & No & $11(20.4 \%)$ & $8(22.9 \%)$ & $3(15.8 \%)$ & & \\
\hline \multirow[t]{2}{*}{ Hydrocephalus } & Yes & $6(11.1 \%)$ & $5(14.3 \%)$ & $1(5.3 \%)$ & \multirow[t]{2}{*}{0.302} & \\
\hline & No & 48 (88.9\%) & $32(85.7 \%)$ & $18(94.7 \%)$ & & \\
\hline \multirow{6}{*}{$\begin{array}{l}\text { Marshall's } \\
\text { Classification }\end{array}$} & Diffuse injury I & $1(1.9 \%)$ & $1(2.9 \%)$ & $0(0.0 \%)$ & \multirow[t]{6}{*}{0.202} & \\
\hline & Diffuse injury II & $26(48.1 \%)$ & $17(48.6 \%)$ & 9 (47.4\%) & & \\
\hline & \begin{tabular}{|l|l} 
Diffuse injury III \\
\end{tabular} & $11(20.4 \%)$ & $7(20.0 \%)$ & $4(21.1 \%)$ & & \\
\hline & Diffuse injury IV & 4 (7.4\%) & $4(11.4 \%)$ & $0(0.0 \%)$ & & \\
\hline & Evacuated lesion & $7(13.0 \%)$ & $2(5.7 \%)$ & 5 (26.3\%) & & \\
\hline & Non-evacuated lesion & $5(9.3 \%)$ & $4(11.4 \%)$ & $1(5.3 \%)$ & & \\
\hline \multirow[t]{5}{*}{ Resident Level } & R2 & $15(28.8 \%)$ & $13(39.4 \%)$ & $2(10.5 \%)$ & \multirow[t]{5}{*}{0.005} & \\
\hline & R3 & $13(25.0 \%)$ & $8(24.2 \%)$ & $5(26.3 \%)$ & & \\
\hline & R4 & $15(28.8 \%)$ & 4 (12.1\%) & $11(57.9 \%)$ & & \\
\hline & R5 & $7(13.5 \%)$ & $6(18.2 \%)$ & $1(5.3 \%)$ & & \\
\hline & R6 & $2(3.8 \%)$ & $2(6.1 \%)$ & $0(0.0 \%)$ & & \\
\hline
\end{tabular}

External ventricular drain (EVD); Confidence interval (CI);

$\S$ Also called Evan's index 
Table 4: Multivariate logistic regression model results of factors affecting external ventricular drain (EVD) accuracy

Multivariate logisitc regression model

\begin{tabular}{|c|c|c|c|c|c|c|c|c|c|}
\hline & & \multirow[b]{2}{*}{$B$} & \multirow[b]{2}{*}{ Std. Error } & \multirow[b]{2}{*}{ Wald } & \multirow[b]{2}{*}{ df } & \multirow[b]{2}{*}{ Sig. } & \multirow[b]{2}{*}{$\operatorname{Exp}(B)$} & \multicolumn{2}{|c|}{$\begin{array}{l}\text { 95\% Confidence Interval for } \\
\operatorname{Exp}(\mathrm{B})\end{array}$} \\
\hline \multicolumn{2}{|c|}{ EVD Tip Accuracy } & & & & & & & Lower Bound & Upper Bound \\
\hline \multirow[t]{8}{*}{ Optimal } & Intercept & .177 & 2.930 & .004 & 1 & .952 & & & \\
\hline & Inserted_by & -.138 & .294 & .221 & 1 & .638 & .871 & .489 & 1.550 \\
\hline & Marshall_class & -.133 & .395 & .112 & 1 & .737 & .876 & .403 & 1.901 \\
\hline & Midline_shift & -.037 & .169 & .048 & 1 & .826 & .963 & .691 & 1.343 \\
\hline & $\mathrm{HCP}_{-}$ratio & -.570 & 11.341 & .003 & 1 & .960 & .566 & $1.256 \mathrm{E}-010$ & $2.546 \mathrm{E}+9$ \\
\hline & Ventricle_size & -.020 & .158 & .017 & 1 & .897 & .980 & .719 & 1.335 \\
\hline & Bicaudate_index & 7.153 & 7.731 & .856 & 1 & .355 & 1277.861 & .000 & $4.866 E+9$ \\
\hline & [EVD_placment=1] & 3.035 & 1.173 & 6.695 & 1 & .010 & 20.811 & 2.088 & 207.426 \\
\hline
\end{tabular}

Hydrocephalus (HCP) 
Table 5: The accuracy of external ventricular drain (EVD) procedure

Table: Accuracy of EVD Procedure

\begin{tabular}{|c|c|c|c|c|}
\hline & & \multicolumn{2}{|c|}{ EVD Placement Technique } & \multirow{2}{*}{$\begin{array}{l}P \\
\text { Value }\end{array}$} \\
\hline & & Classic & Navigation & \\
\hline \multirow{3}{*}{$\begin{array}{l}\text { Accuracy-tip } \\
\text { (Kakarla grade) }\end{array}$} & Grade 1 (Optimal \& Adequate) & $20(57.1 \%)$ & $18(94.7 \%)$ & \multirow[t]{3}{*}{0.009} \\
\hline & Grade 2 (Suboptimal, in non-eloquent area) & $2(5.7 \%)$ & $1(5.3 \%)$ & \\
\hline & Grade 3 (Suboptimal, in eloquent area) & $13(37.1 \%)$ & $0(0.0 \%)$ & \\
\hline \multirow{3}{*}{$\begin{array}{l}\text { Accuracy- } \\
\text { entry point }\end{array}$} & Ideal (On coronal suture or anterior, \& Lateral to midline $>2.5 \mathrm{~cm})$ & $23(65.7 \%)$ & $14(73.7 \%)$ & \multirow[t]{3}{*}{0.309} \\
\hline & Suboptimal $(1 \mathrm{~cm}-2.5 \mathrm{~cm}$ lateral to midline) & $8(22.9 \%)$ & $5(26.3 \%)$ & \\
\hline & Potentially dangerous (Posterior to coronal sutures or within $1 \mathrm{~cm}$ form midline) & $4(11.4 \%)$ & $0(0.0 \%)$ & \\
\hline \multirow[t]{2}{*}{ Safe EVD Path } & Yes & $15(42.9 \%)$ & $16(84.2 \%)$ & \multirow[t]{2}{*}{0.03} \\
\hline & No & $20(57.1 \%)$ & $3(15.8 \%)$ & \\
\hline
\end{tabular}

External ventricular drain (EVD)

Highlighted cells: Primary ouctome 
Table 6: Clinical outcome of external ventricular drain (EVD) procedure

Table: Clinical outcome in patients who had EVD placement procedure

EVD Placement Technique

\begin{tabular}{|c|c|c|c|c|c|c|}
\hline & & Entire Cohort & Freehand-Classic & Navigation-Guided & P Value & $95 \% \mathrm{Cl}$ \\
\hline \multicolumn{2}{|l|}{ Mean no. of Passes } & $1.43 \pm 0.75$ & $1.63 \pm 0.88$ & $1.16 \pm 0.38$ & 0.018 & $(-0.86,-0.09)$ \\
\hline \multicolumn{2}{|l|}{ EVD stay (days) } & $6.2 \pm 4.0$ & $6.5 \pm 4.2$ & $5.8 \pm 3.8$ & 0.593 & $(-2.9,1.6)$ \\
\hline \multicolumn{2}{|l|}{ ICU Stay (days) ${ }^{\S}$} & $19.6 \pm 8.4$ & $20.0 \pm 8.8$ & $19.1 \pm 8.1$ & 0.809 & $(-7.6,6.0)$ \\
\hline \multicolumn{2}{|l|}{ Total Hospital Stay (days) } & $39.4 \pm 48.3$ & $45.1 \pm 57.0$ & $28.7 \pm 23.2$ & 0.141 & $(-38.6,5.7)$ \\
\hline \multicolumn{2}{|l|}{ Intubation (days) ${ }^{\S}$} & $14.0 \pm 7.0$ & $14.4 \pm 6.9$ & $13.2 \pm 7.3$ & 0.666 & $(-7.2,4.7)$ \\
\hline \multirow[t]{2}{*}{ EVD Malfunction } & Yes & 14 (26.9\%) & $11(33.3 \%)$ & $3(15.8 \%)$ & \multirow[t]{2}{*}{0.17} & \\
\hline & No & $38(73.1 \%)$ & $22(66.7 \%)$ & $16(84.2 \%)$ & & \\
\hline \multirow[t]{2}{*}{ Revision } & Yes & $7(13.5 \%)$ & $6(18.2 \%)$ & $1(5.3 \%)$ & \multirow[t]{2}{*}{0.189} & \\
\hline & No & 45 (86.5\%) & $27(81.8 \%)$ & $18(94.7 \%)$ & & \\
\hline \multirow[t]{3}{*}{ CSF Infection } & No Infection & 40 (85.1\%) & $25(89.3 \%)$ & $15(78.9 \%)$ & \multirow[t]{3}{*}{0.334} & \\
\hline & Contamination & 4 (8.5\%) & $1(3.6 \%)$ & $3(15.8 \%)$ & & \\
\hline & Infection (Treated) & $3(6.4 \%)$ & $2(7.1 \%)$ & $1(5.3 \%)$ & & \\
\hline \multirow[t]{2}{*}{ Radiological Complication } & Yes & $11(20.4 \%)$ & $8(22.9 \%)$ & $3(15.8 \%)$ & \multirow[t]{2}{*}{0.538} & \\
\hline & No & 43 (79.6\%) & $27(77.1 \%)$ & $16(84.2 \%)$ & & \\
\hline \multirow[t]{4}{*}{ Discharge Status } & Home & 1 (1.9\%) & $0(0.0 \%)$ & $1(5.3 \%)$ & \multirow[t]{4}{*}{0.527} & \\
\hline & Rehab. Center & 15 (27.8\%) & $9(25.7 \%)$ & $6(31.6 \%)$ & & \\
\hline & Long-Term Care & 10 (18.5\%) & $7(20.0 \%)$ & $3(15.8 \%)$ & & \\
\hline & \begin{tabular}{|l} 
Death \\
\end{tabular} & 28 (51.9\%) & $19(54.3 \%)$ & $9(47.4 \%)$ & & \\
\hline
\end{tabular}

Confidence interval (CI); External ventricular drain (EVD); Intensive care unit (ICU); Cerebrospinal fluid (CSF).

Highlighted cells: Primary ouctome

$\S$ Excluding patients whose management was changed to "comfort measures" and eventually died during the hospitalisation 
FIGURES 
Figure 1: Different steps in applying the navigation system to guide the ventricular catheter placement

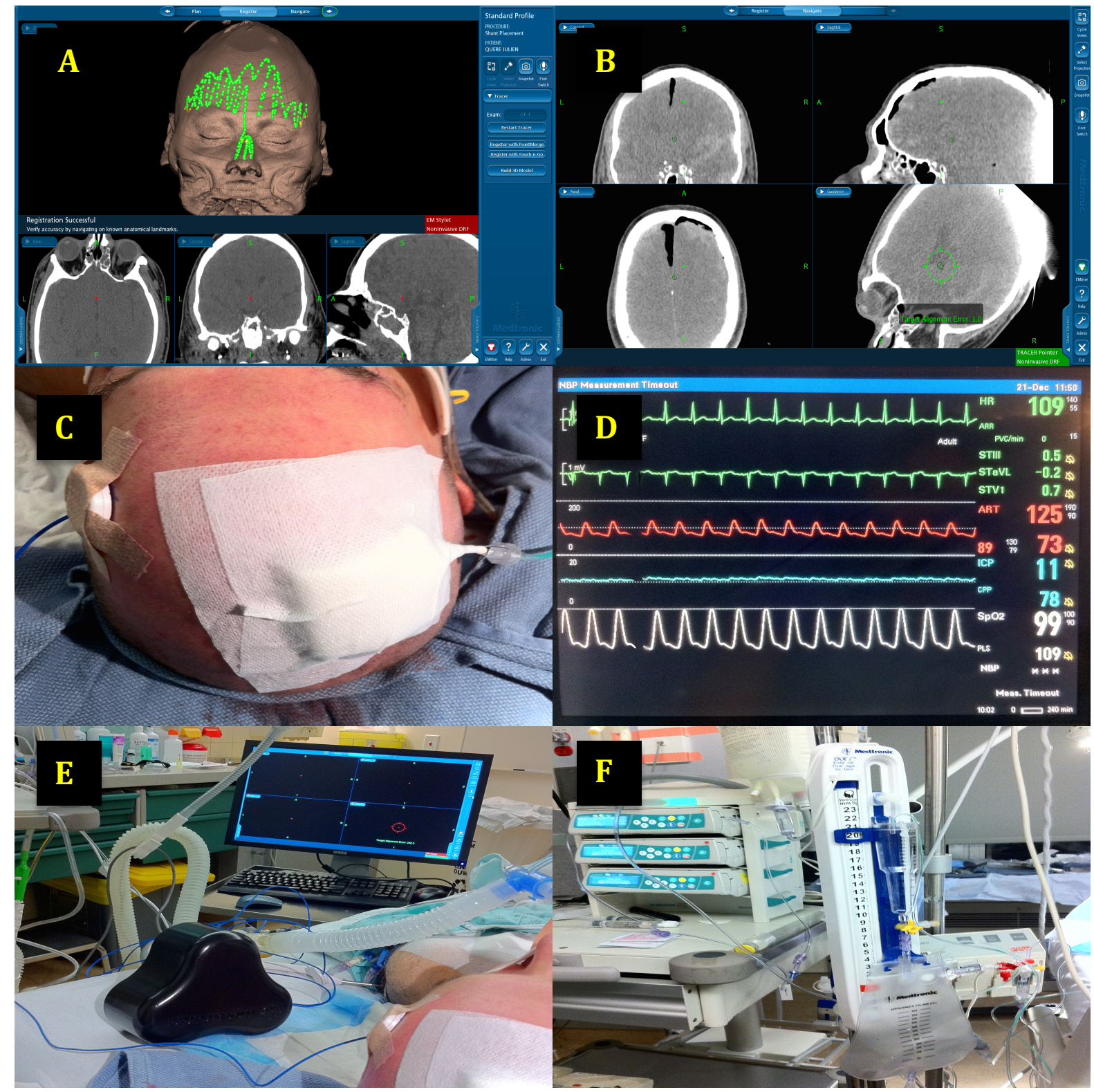

Different steps in applying the navigation system. A: Surface registration. B: Guiding the catheter to the preselected target. C: Non-invasive tracker and dressing application over the already placed drain. D: Vital signs monitor showing ICP in light green. E: Setting of different parts at the end of procedure. F: Ventricular cerebrospinal fluid collecting bag with pressure monitor column. 


\section{Figure 2: Different measurements applied to pre-procedural scan}
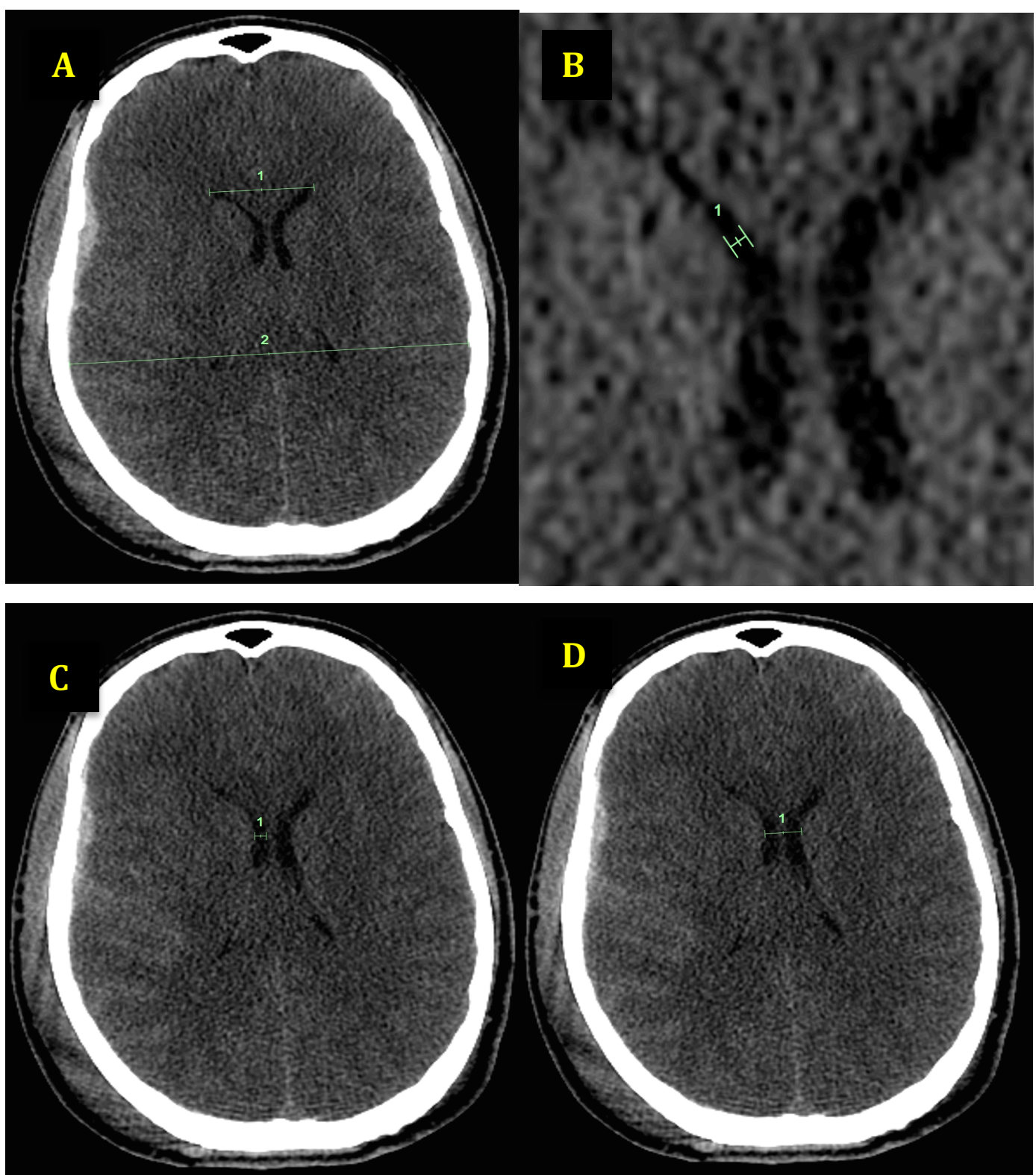

Axial computed tomography images showing different measurements used to evaluate scans prior to ventricle catheter insertion. A: Hydrocephalus ratio (the ratio of maximum width of the frontal horns to the maximum width of the inner table of the cranium) and Bifrontal ventricular width (the maximum width of the frontal horns). B: Ventricular size (the width of the ipsilateral frontal horn calculated with a line tangential to the caudate nucleus). C: Caudate-septal line (in the ipsilateral side, the length form a tangential line to the caudate nucleus to the septum pellucidum in the midline). D: Bicaudate width (the maximum width between two lines drown tangential to both caudate nuclei and perpendicular to the septum pellucidum in the midline), and Bicudate index (the ratio of the width of both lateral ventricles at the level of the head of caudate nuclei to the maximum width of the inner tables of the cranium at the same axial level). 
Figure 3: Midline shift measurement

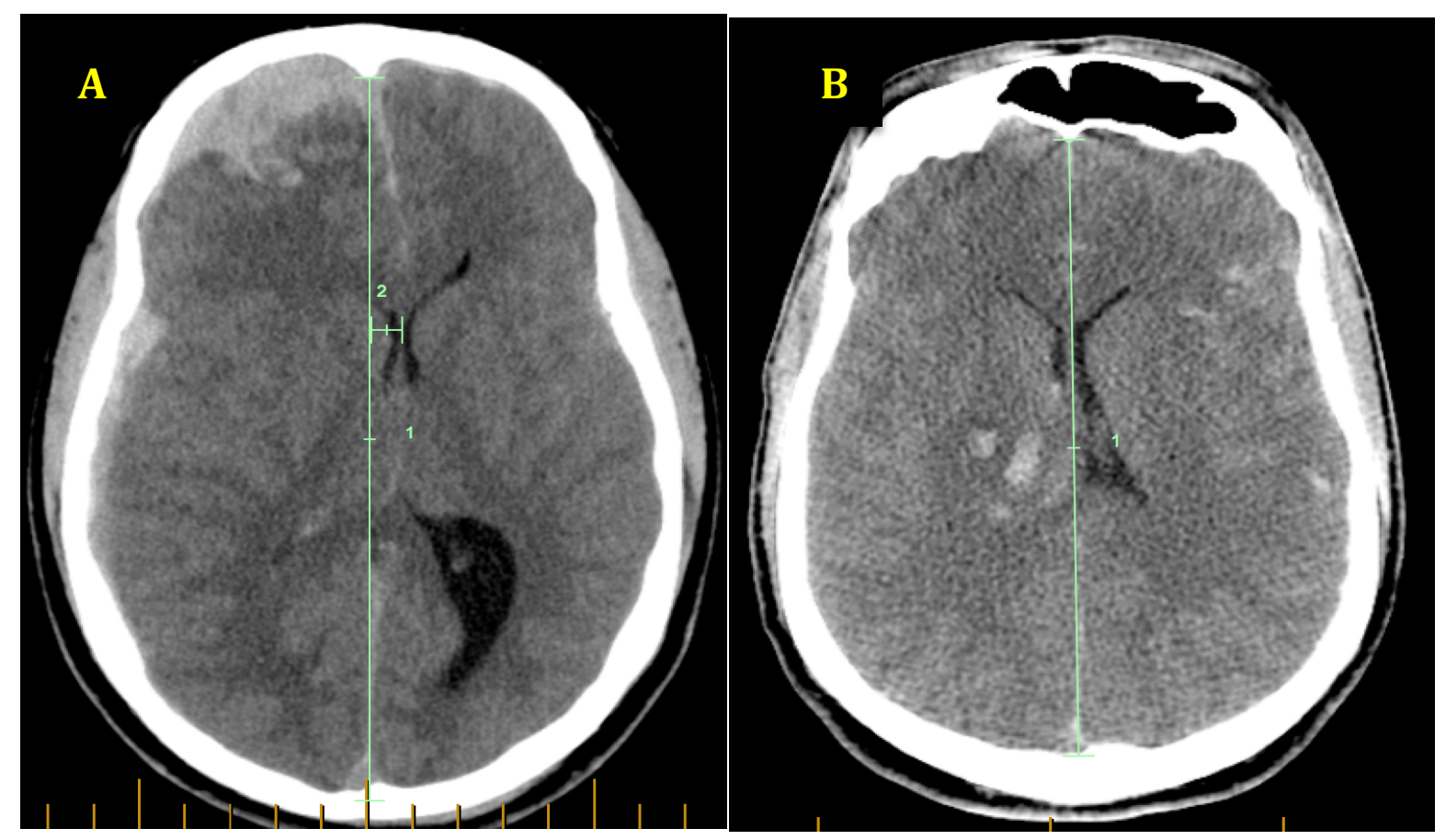

Axial computed tomography images demonstrating the midline shift measurement using a crude method of measuring the width of the maximum deviation of septum pellucidum from a line sketched between the far anterior and the far posterior leaflets of the falx. A: An adult patient with head trauma and $7 \mathrm{~mm}$ midline shift who was taken for urgent surgery. B: An adult patient with head trauma and no midline shift who had ventricular catheter inserted initially and later on taken for surgery because of refractory high intracranial pressure. 
Figure 4: Catheter length using the cranium outer table

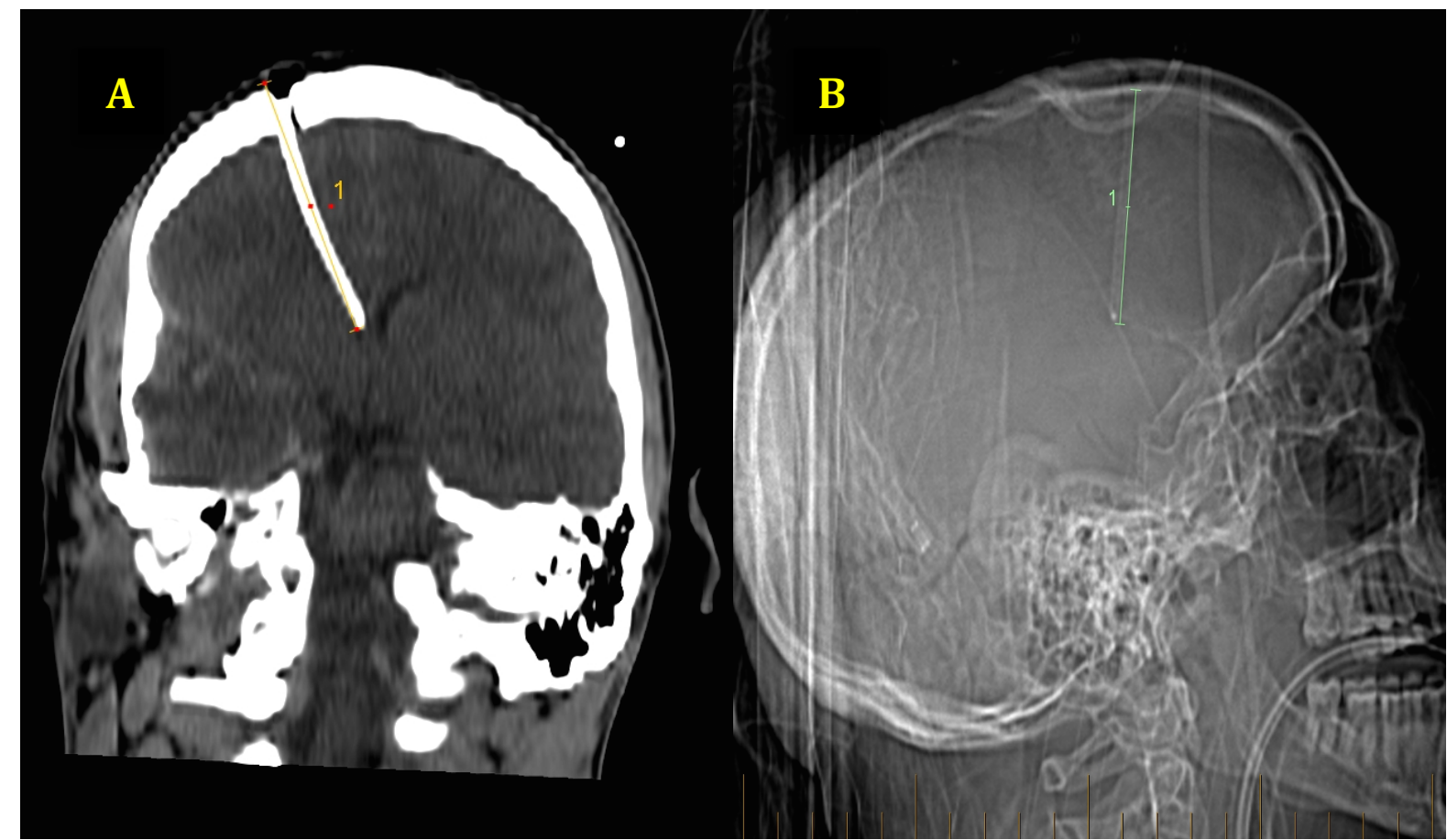

Computed tomography images showing the measurements of intracranial ventricular catheter length. A: Coronal image reformatted obliquely and centered at the catheter. In our study, the calculated length is from the tip of the catheter to the burr hole at the cranium outer table. B: Scout image as used by other studies calculating the catheter length to the burr hole at the cranium inner table. 
Figure 5: The steps of recruitment

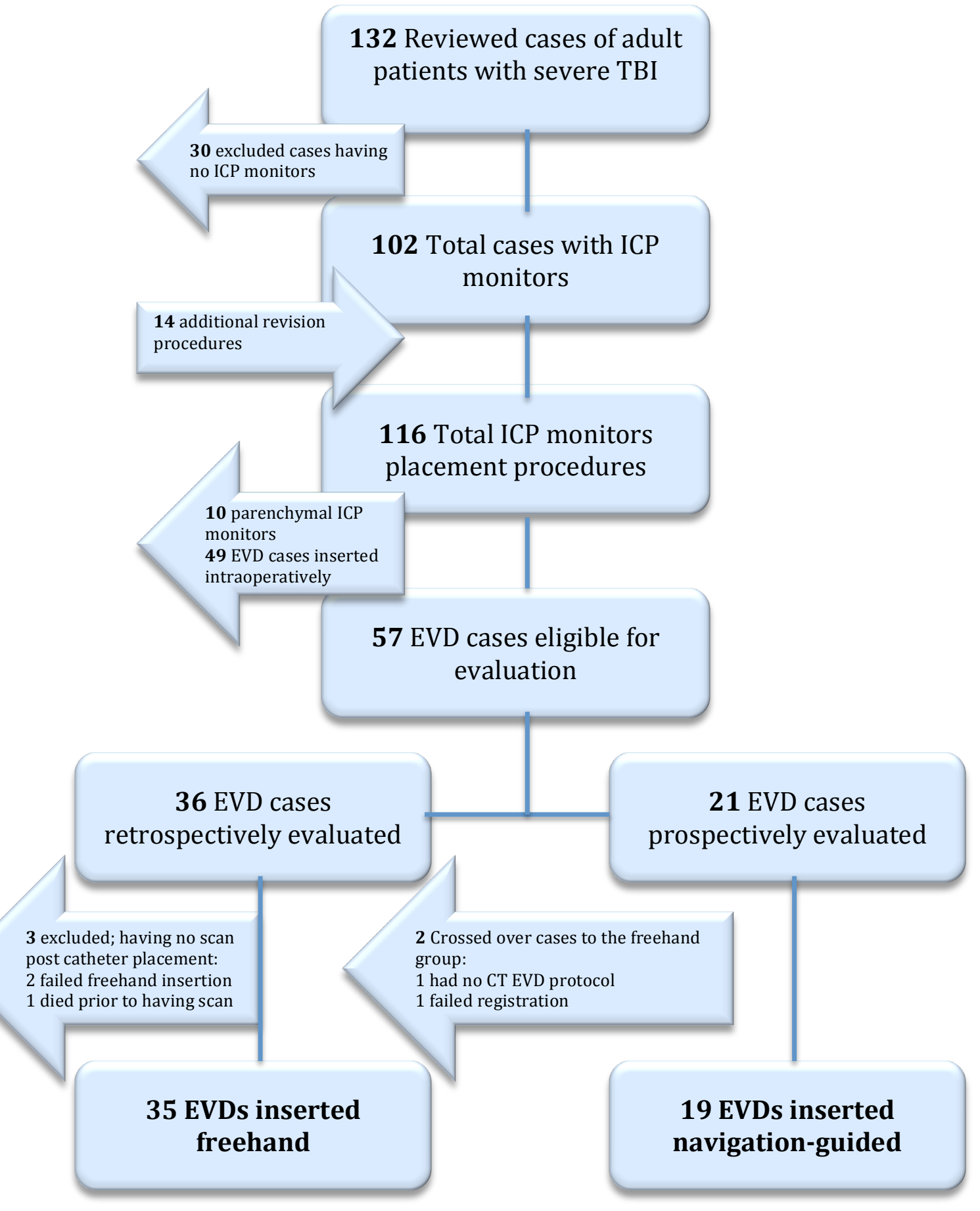

Flow chart of the steps of recruitment in our study. 
Figure 6: Example of cases that got no intracranial pressure (ICP) pressure monitors

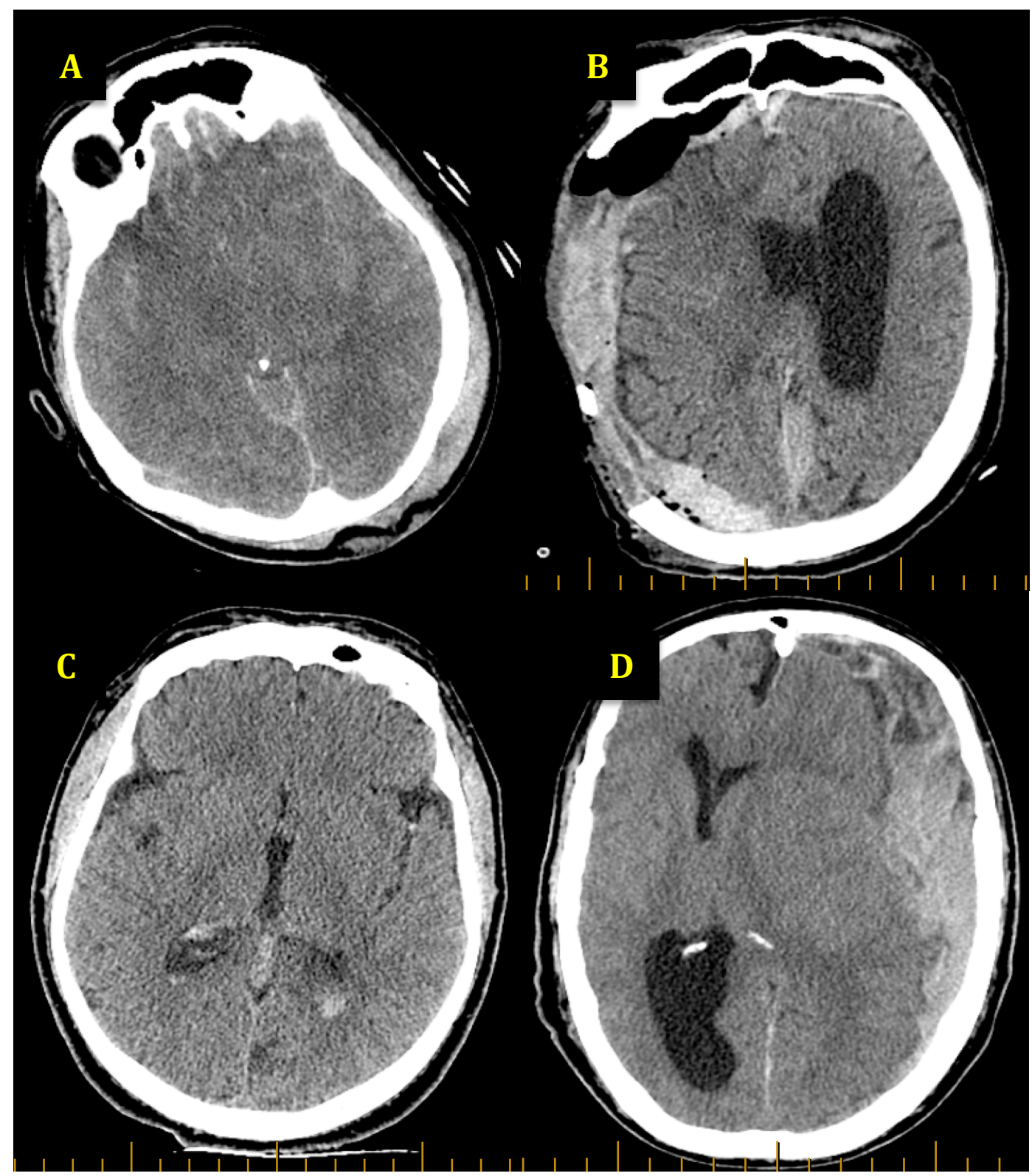

Axial computed tomography images showing example of cases with severe head injury that had no intracranial pressure monitor inserted. A: A case with evolving brain stem death clinically and loss of white grey differentiation in the scan. B: An elderly with moribund injury and who sustained re-bleeding again after surgery. $\mathbf{C}$ : A middle age male who had modest clinical improvement in the initial hours after admission and was clinically examinable. D: An elderly with poor neurological status who was taken for urgent surgery and remained with guarded prognosis. 
Figure 7: Different intracranial pressure (ICP) monitors

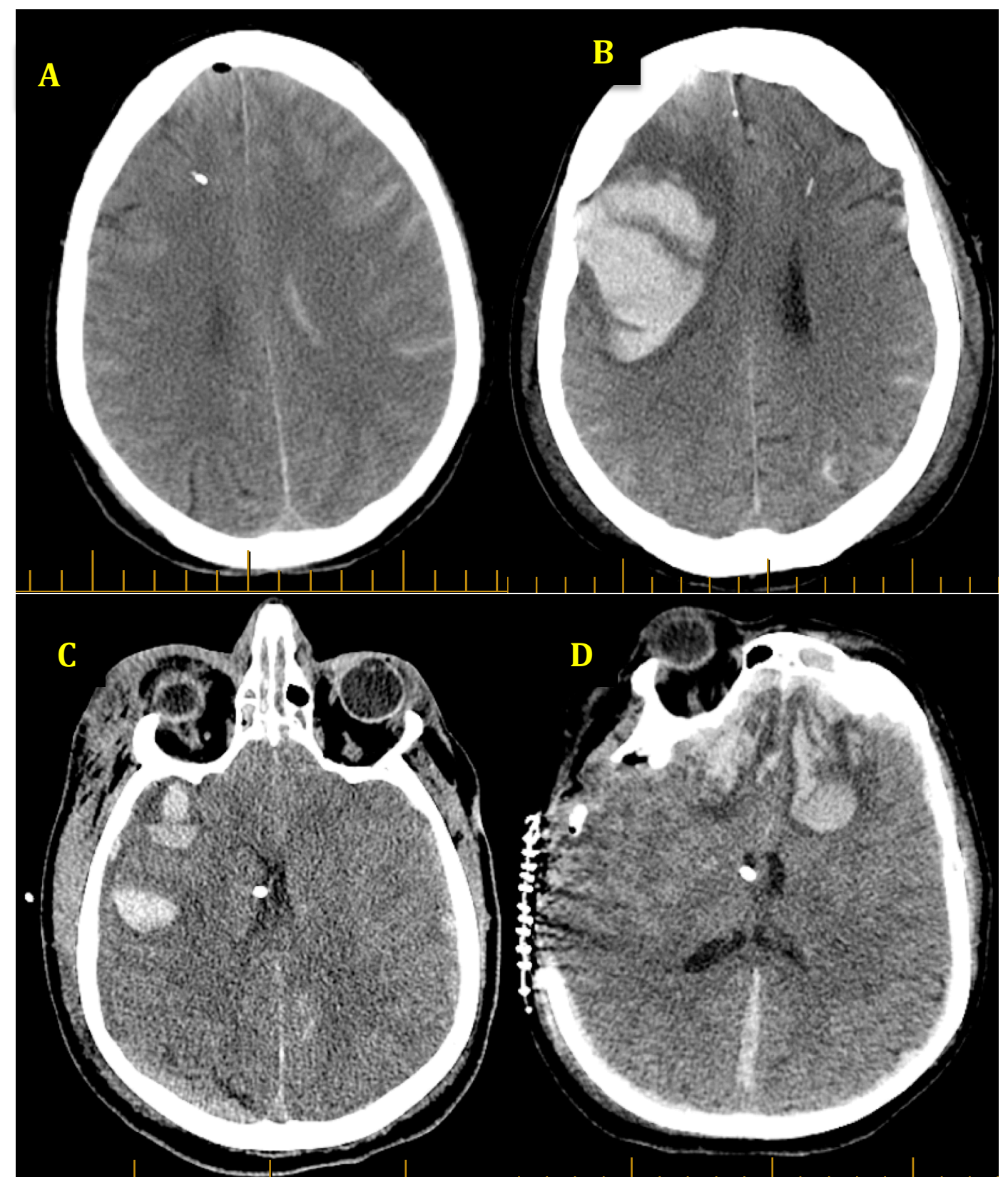

Axial computed tomography images showing two different modalities used for intracranial pressure monitoring. A \& B: Parenchymal pressure monitor (Codman Microsensor ICP Transducer). C \& D: Intraventricular-placed monitor (Medtronic EDM Ventricular Catheter). 
Figure 8: Two cases that crossed over to the freehand group
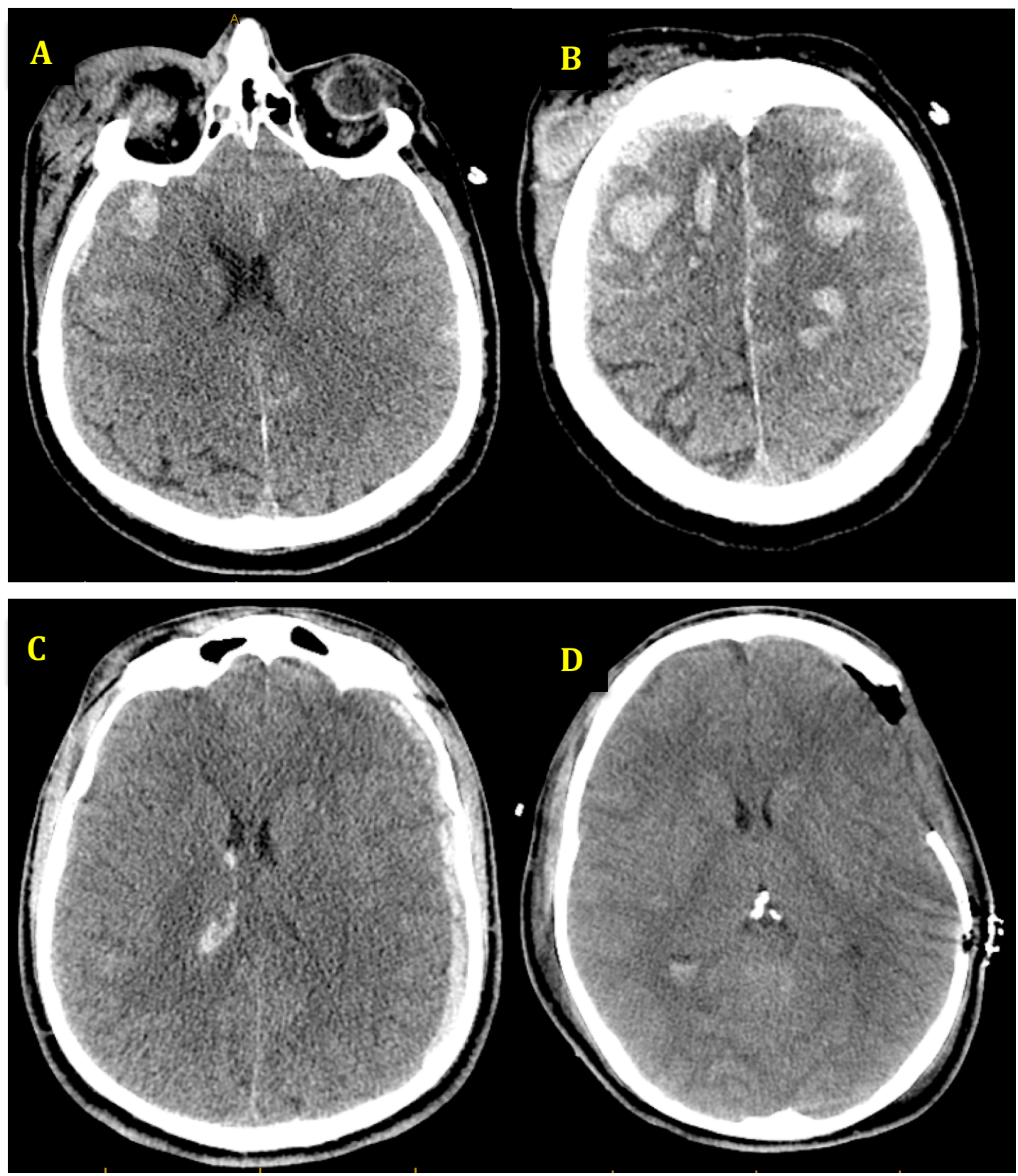

Axial computed tomography images of the two cases that crossed over to the freehand group. Repeating the scan was not to be done according to the study protocol unless having clinical indication. A \& B: 66 year old male who sustained head trauma and right forehead and periorbital contusion and subgaleal bleed. The decision for ventricular catheter insertion was made 5 hours after the scan, which was enough time for the subgaleal hematoma to progress and made it difficult to register the scan to the patient head. C \& D: 22 year old male who was operated urgently for subdural hematoma evacuation and removal of bone flap. The postoperative scan was not done according to the CT EVD Protocol, hence, was not applicable for the navigation system. 
Figure 9: Bar chart showing the different intracranial pressure monitors used in severe traumatic brain injury

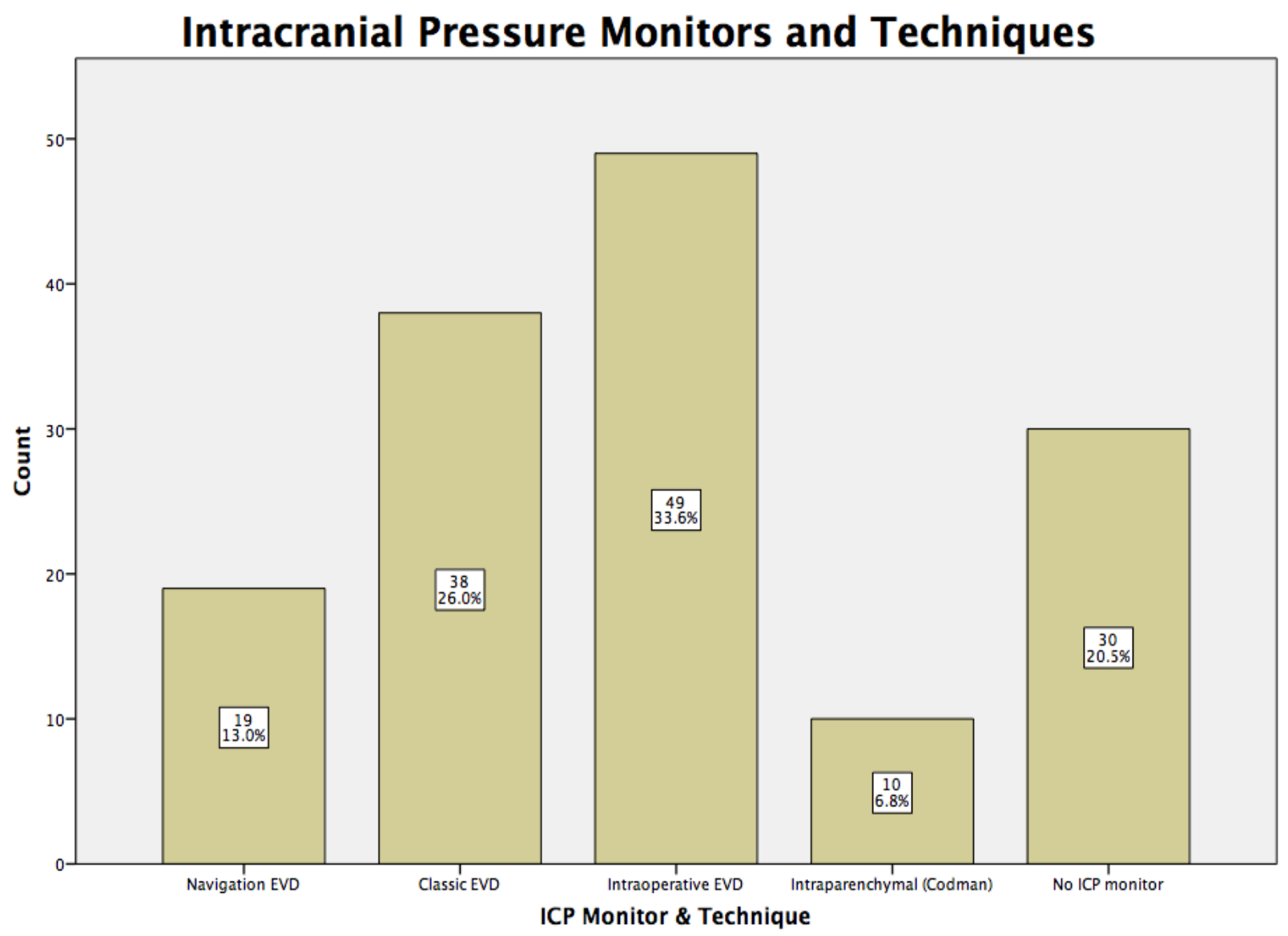

Intracranial pressure monitors (ICP) and techniques used in severe traumatic brain injury in our institution over 2 years. The navigation and the classic EVD groups were the evaluated in our study. Three cases from the classic freehand group were excluded. 
Figure 10: A challenging case managed with navigation guidance
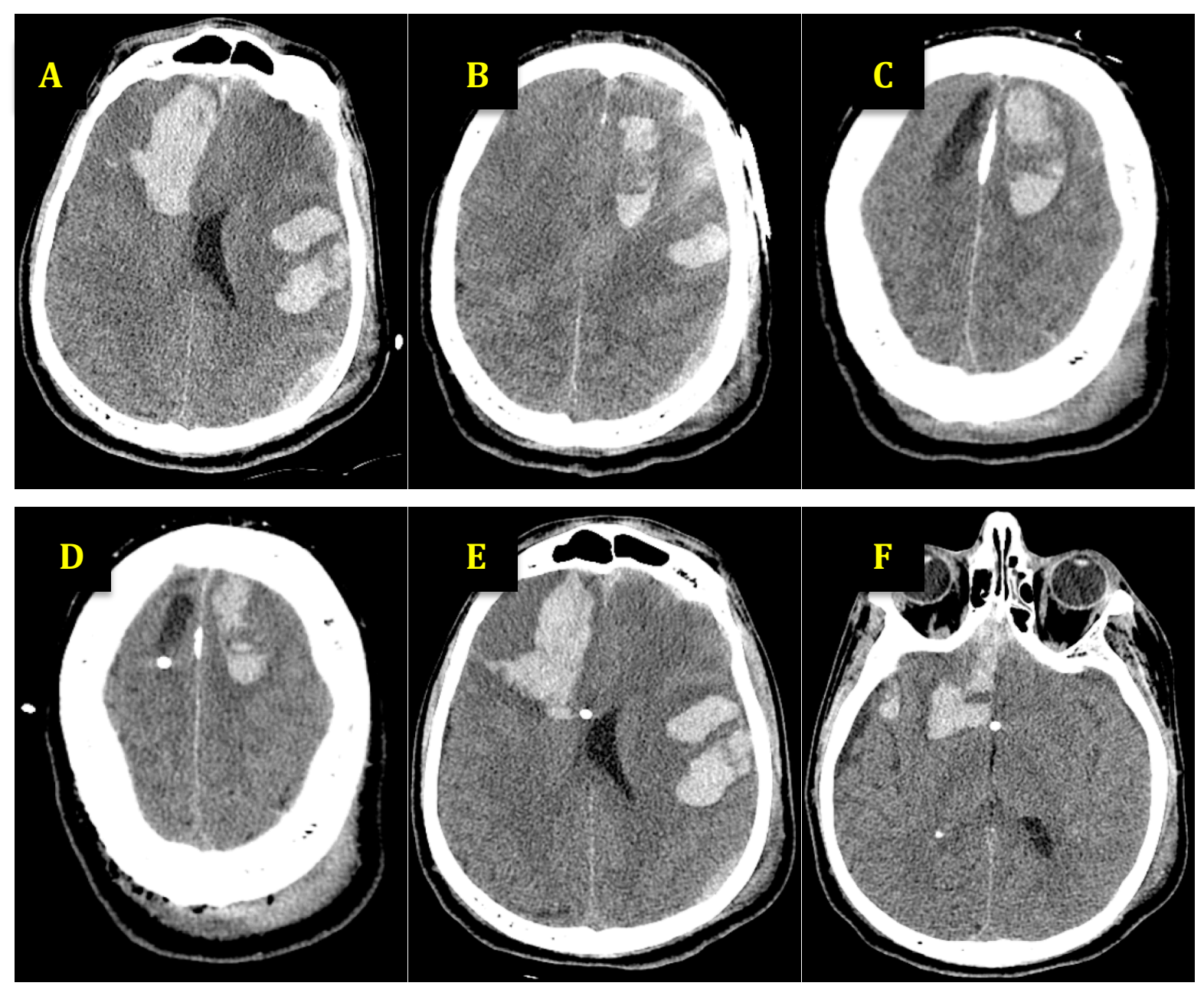

Axial computed tomography images of a challenging case managed with navigation system guidance. A young male with alcohol withdrawal seizure sustained severe traumatic brain injury. Though the right ventricle is effaced, the treating team decision was to place the ventricular catheter in the right side to avoid the multiple cerebral hematomas in the left side while also avoiding the other hematoma in the right side and the encephalomalacic area too in the upper frontal region. The aim was to place the catheter in the third ventricle to be able to drain the contralateral side also (A-C). From the first pass using the navigation the catheter did neither pass through the encephalomalacic area nor through the hematomas and successfully passed through the ipsilateral foramen of Monro into the third ventricle (D-F). 
Figure 11: Bar chart showing the mode of injury for the navigation and freehand groups

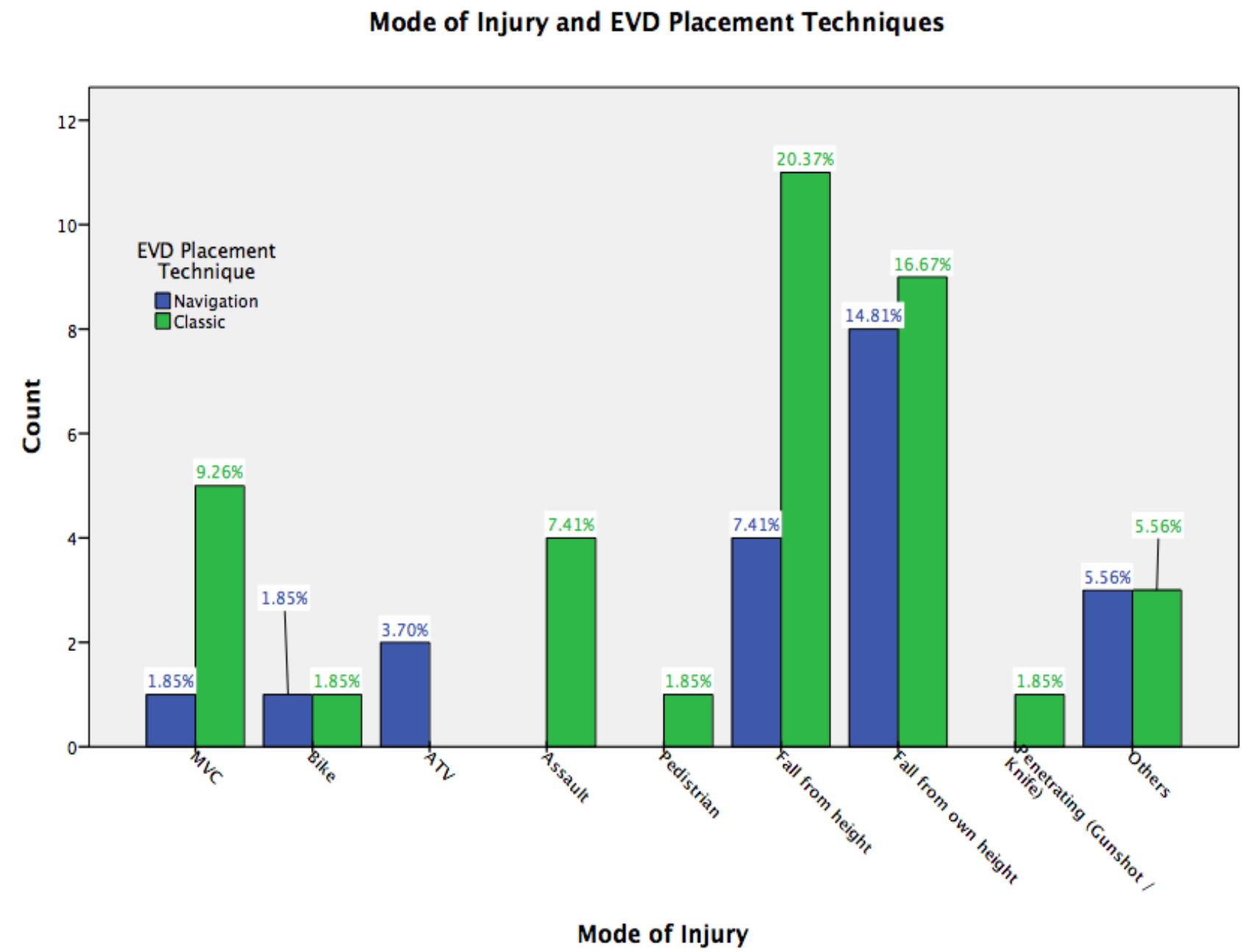

Bar chart showing the mode of injury for the navigation and freehand groups. The falls, either from height and falls from own height level, were the common 2 mechanisms of injury. 
Figure 12: Bar chart showing the GCS after 24 hours for the navigation and freehand groups

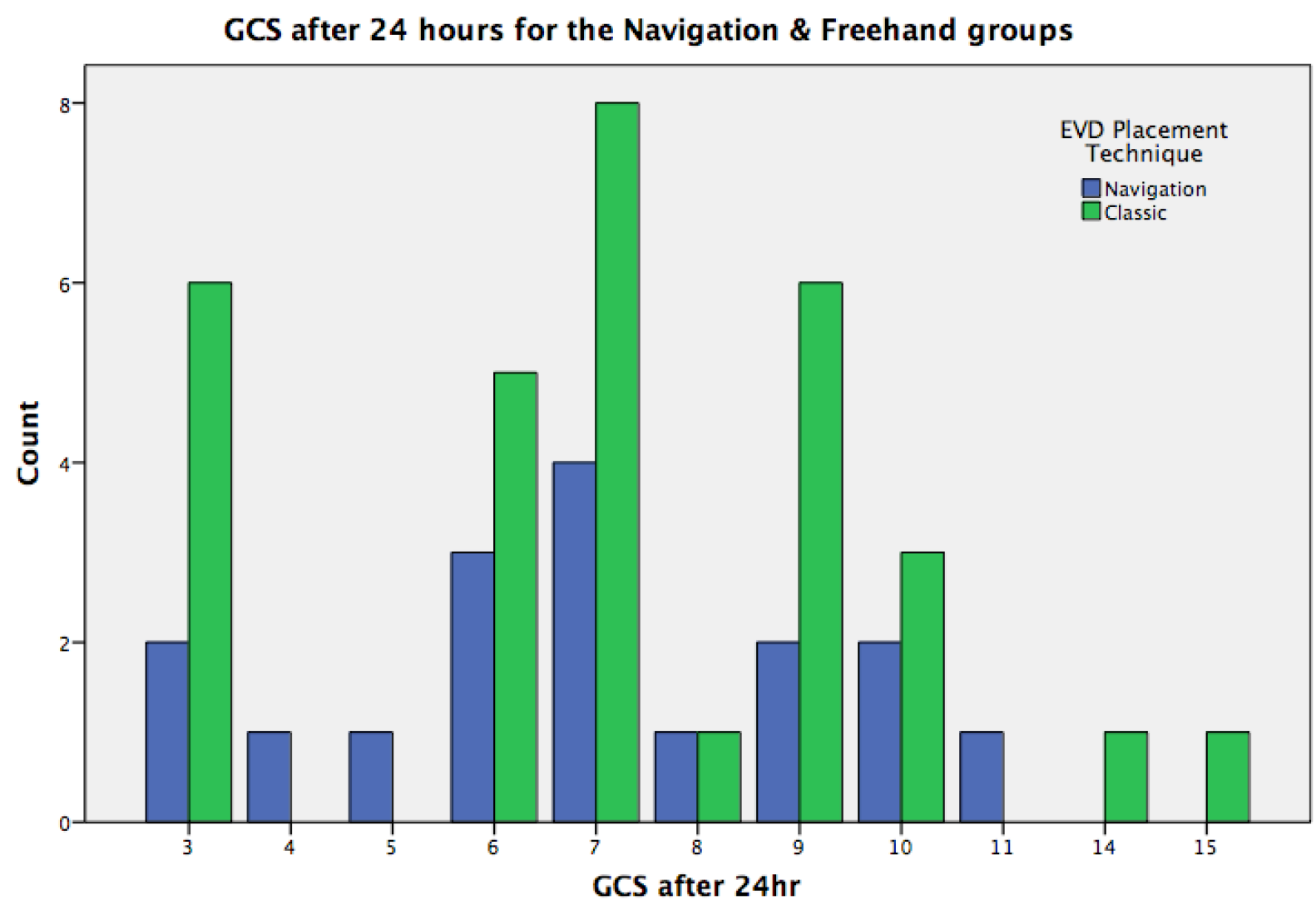

Bar chart showing the GCS after 24 hours for the navigation and freehand groups. Evaluation of GCS after 24 hours may be of a better prognostic value. 
Figure 13: Bar chart showing level of training and catheter placement accuracy

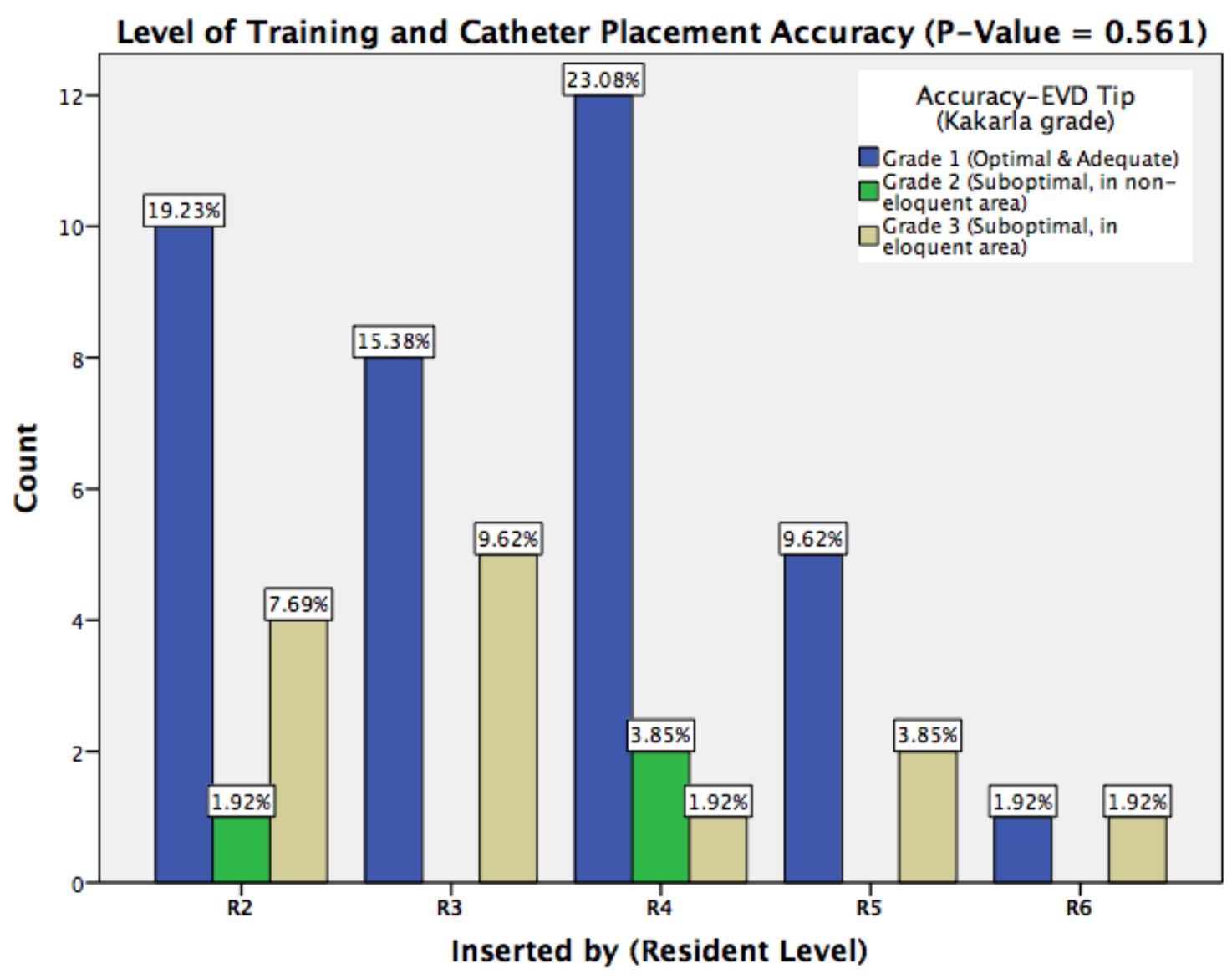

Bar chart showing the association between level of training and catheter placement accuracy. This did not take in account the use of navigation. 
Figure 14: The single case in the navigation group that had the catheter misplaced in the contralateral ventricle

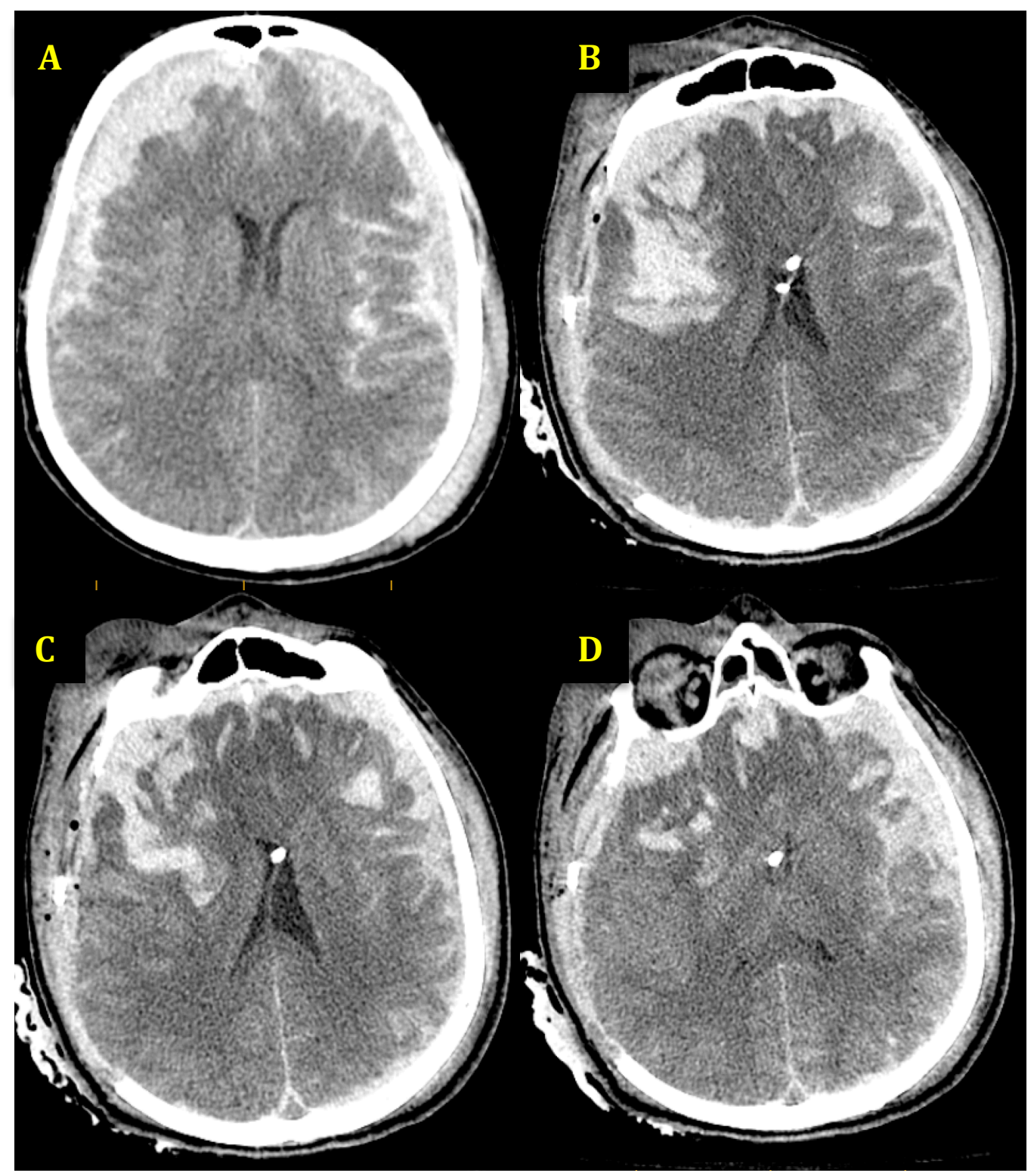

Axial computed tomography images of the single case that had ventricular catheter misplaced into the contralateral frontal horn (grade 2) in the navigation-guided group. A 76 year old male on antiplatelet therapy who sustained head trauma after a fall. He was operated on urgently for subdural hematoma evacuation and removal of bone flap (A). He had the right ventricular catheter inserted intraoperatively and because of catheter malfunction, he had the left catheter inserted under the navigation guidance. Because of the expansion injury post operatively, he had a large intracerebral hemorrhage with septum pellucidum shifted to the contralateral side $(\mathbf{B} \& \mathbf{C})$. The catheter tip ended near the contralateral foramen of Monro (D). 
Figure 15: Example of cases with ventricular catheter tip misplacement



Computed tomography showing examples of ventricular catheter tip misplacement (grade 3) when inserted freehand. A: At the striatum. B: At the basal cisterns. C: At the thalamus, D: At the pons (brainstem). 
Figure 16: Graph showing the number of passes related to the ventricular catheter accuracy

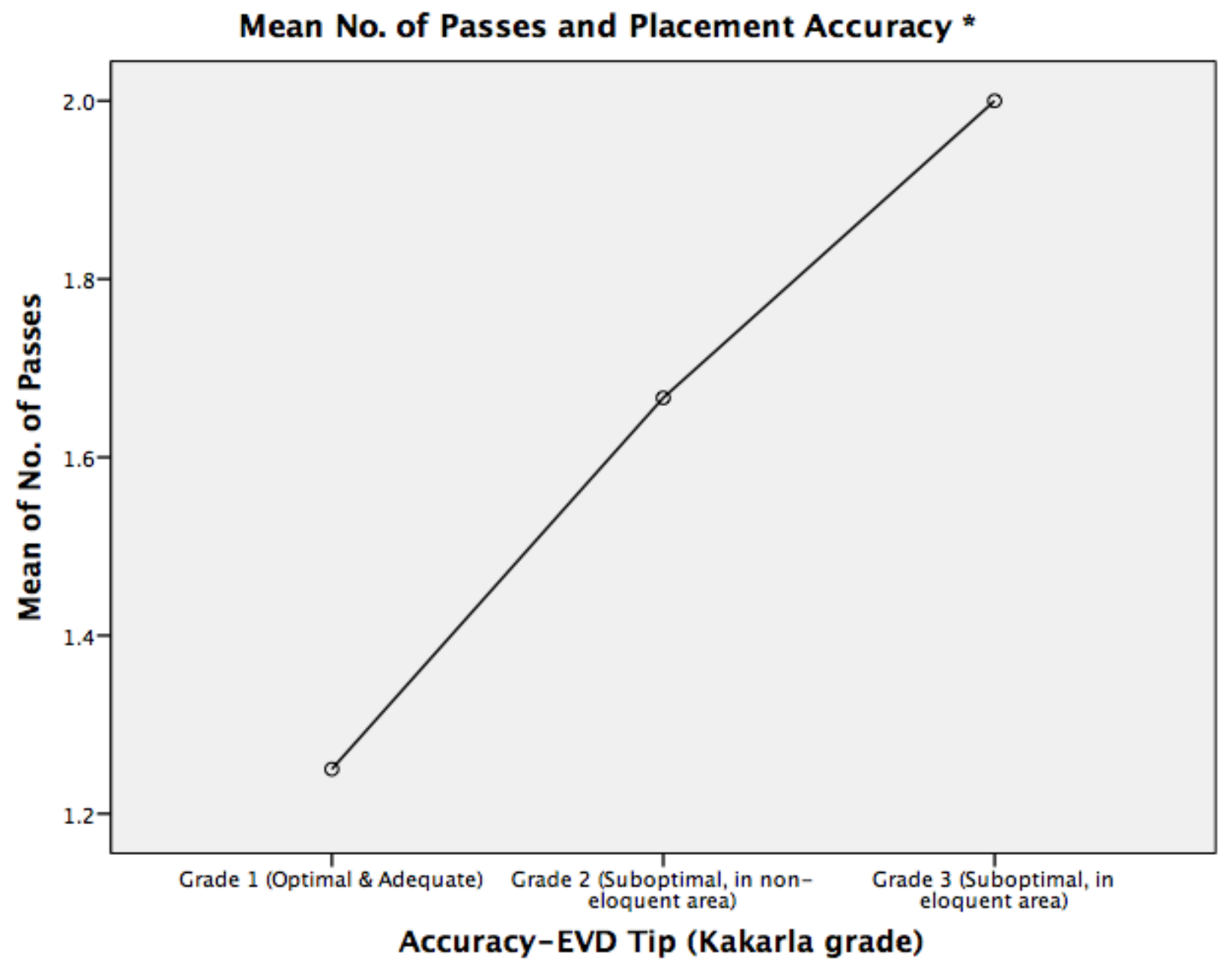

* $P$-Value $=0.009$ (Significant difference between grade $1 \& 3$ )

Graph showing significant difference of mean number of passes between grade 1 accuracy and grade 3. 
Figure 17: Tract hemorrhage related to catheter placement

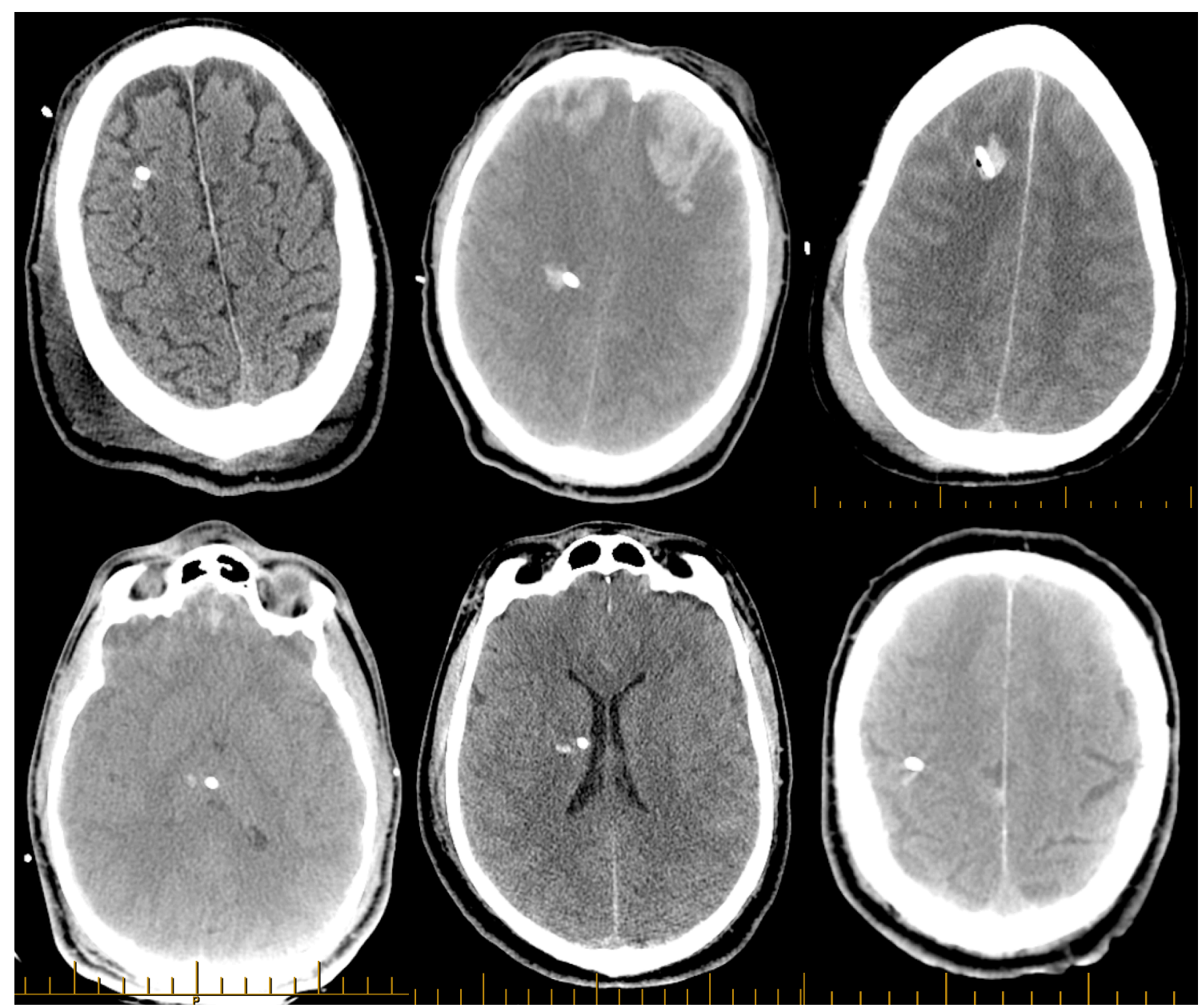

Axial computed tomography images showing hemorrhagic complication related to ventriculostomy catheter insertion. 
Figure 18: Hemorrhagic complications and mean number of passes

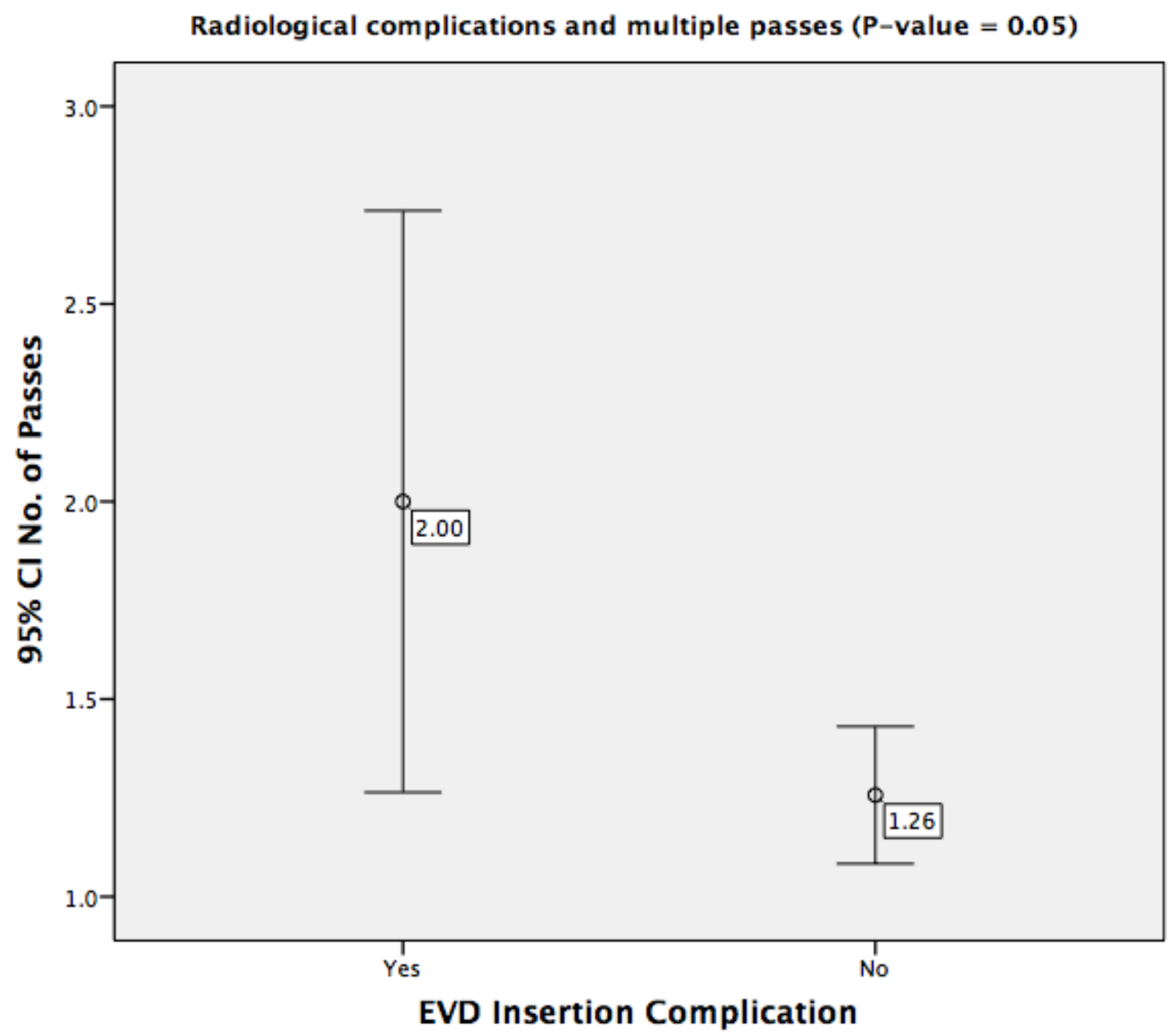

Hemorrhagic complications are associated with higher number of passes. 
Figure 19: Bar chart of the accuracy of the entry point and safe ventricular path

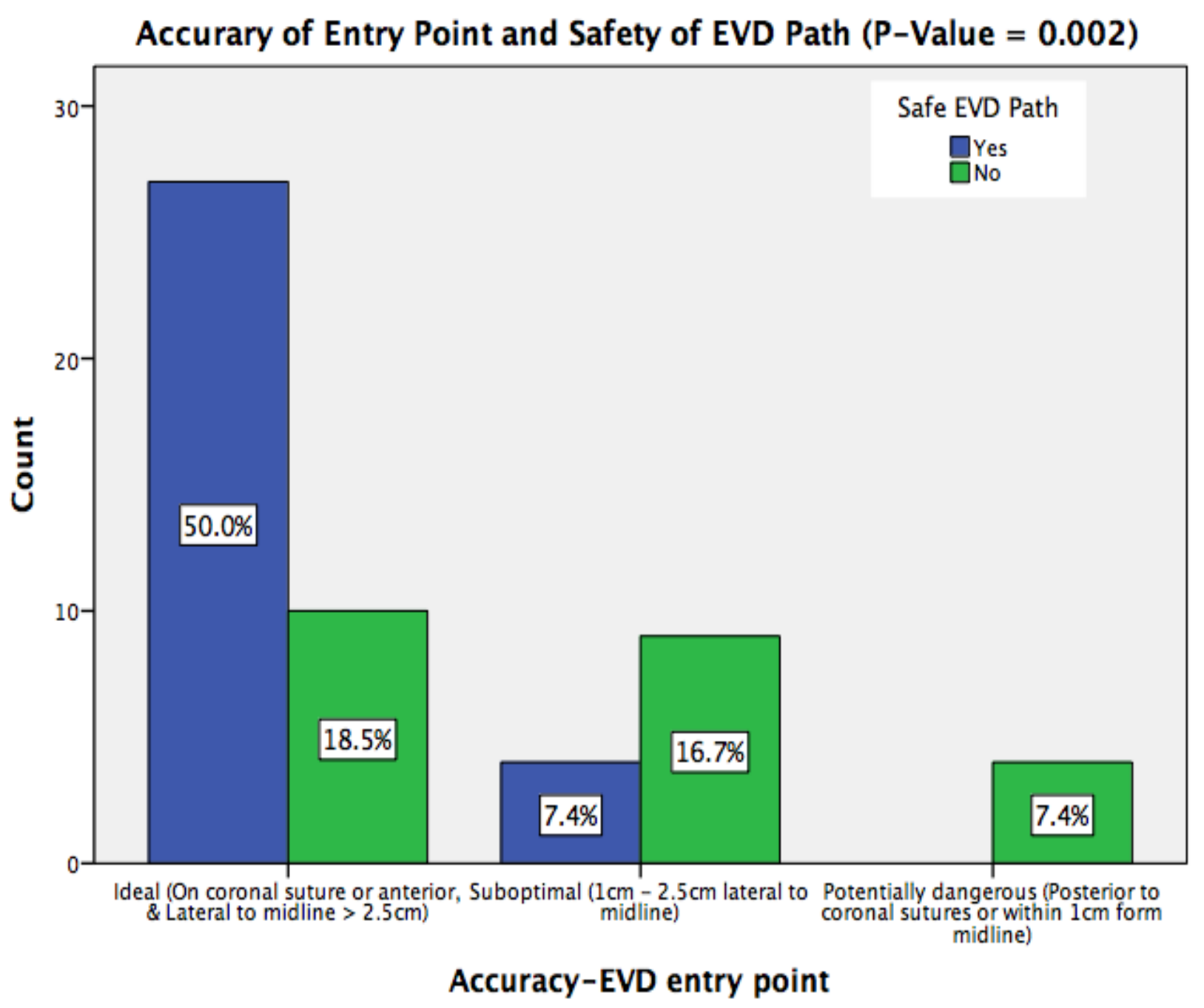

Bar chart showing the association between optimal entry point and safe path during the insertion of ventricular catheter. 
Figure 20: Additional time required when applying the navigation guidance to the external ventricular drain (EVD) insertion procedure

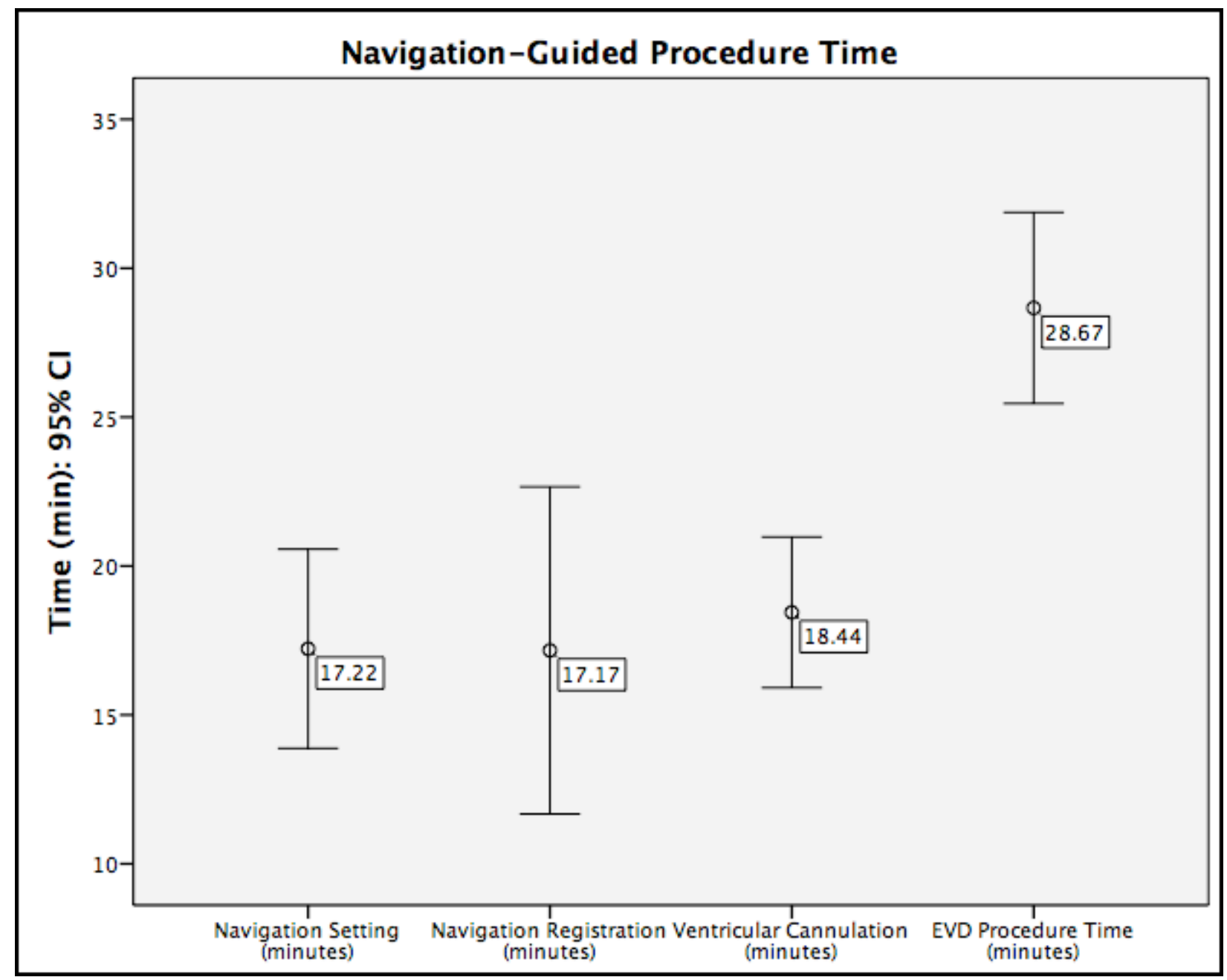

The extra time required for the navigation-guided EVD procedure. The navigation setting and the navigation registration are the additional categories when compared to the classic freehand procedure. 
Figure 21: Placement accuracy relation with ventricular drain length

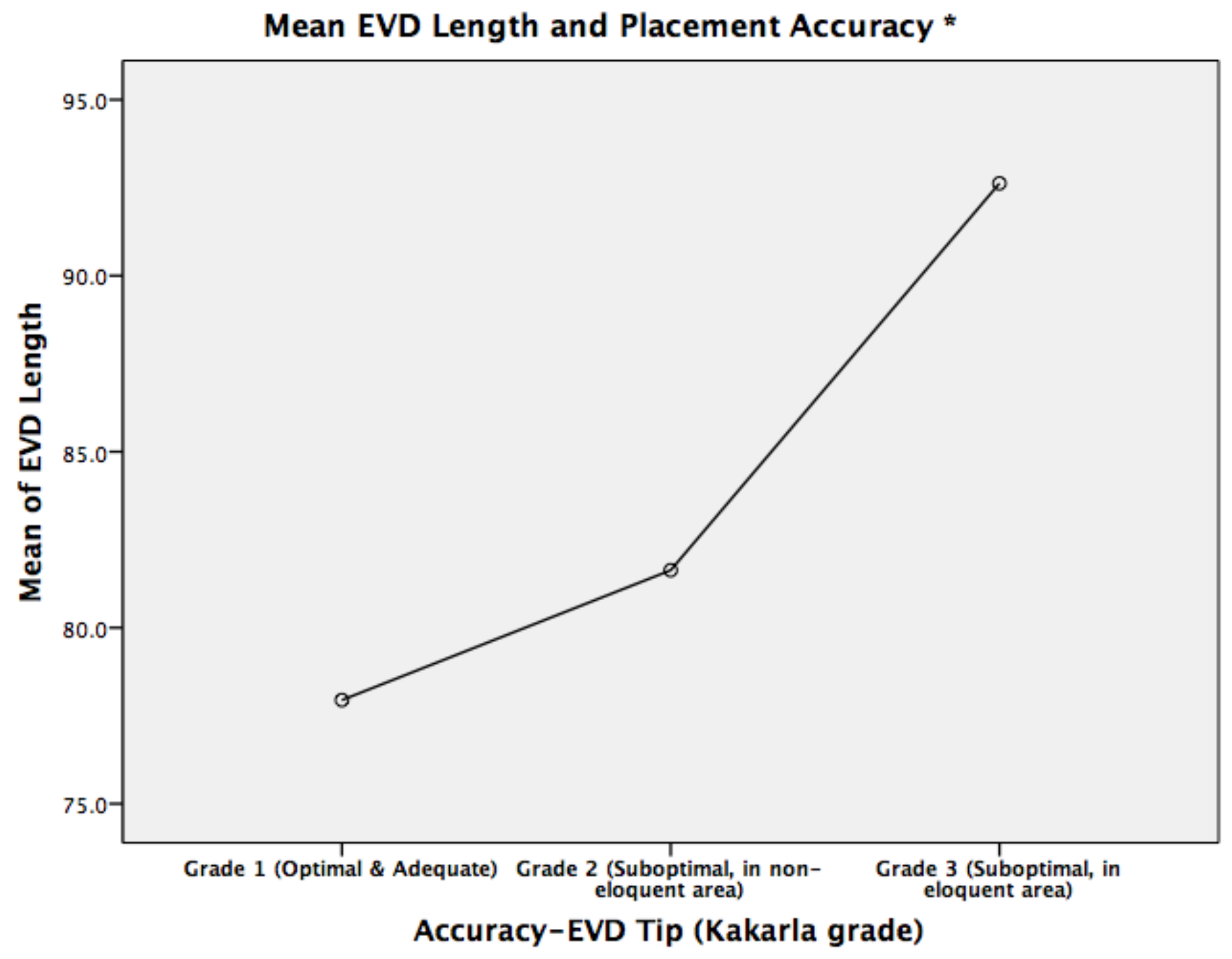

* $P$-Value $<0.000$ (Significant difference between grade $1 \& 3$ )

There was significant association between ventricular catheter length and placement accuracies; grade 1 and grade 3. 
Figure 22: Optimal ventricular catheter insertion

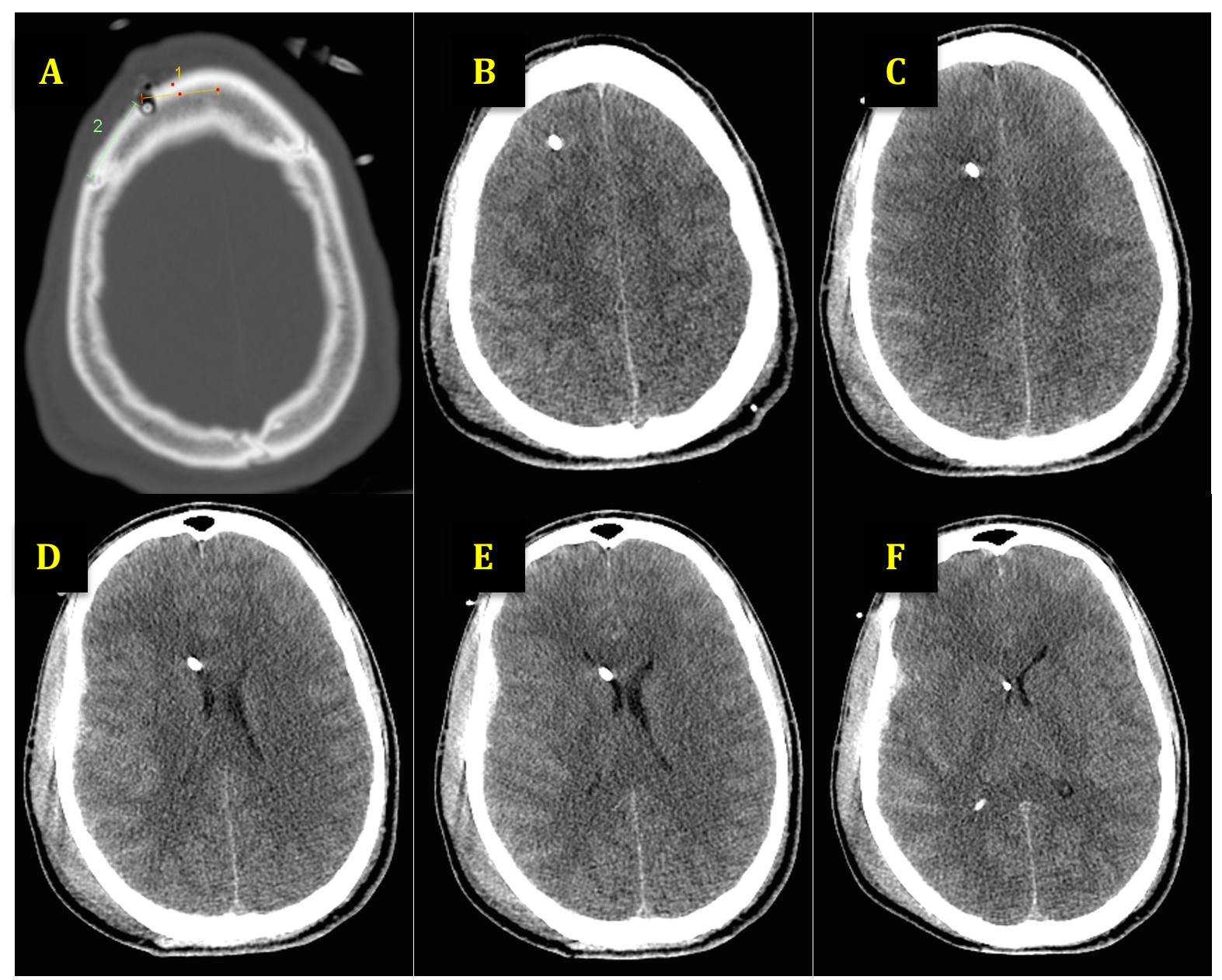

Computed tomography images showing the optimal external ventricular catheter placement and no post-insertion radiological complications. A: At the level of cranium with ideal insertion landmarks away from midline and anterior to the coronal suture. B \& C: The catheter path through the frontal lobe. D \& E: The catheter path into the frontal horn. F: The catheter tip is near the foramen of Monro. 
Figure 23: Ventricular catheter misplacement detected in post-procedural scans
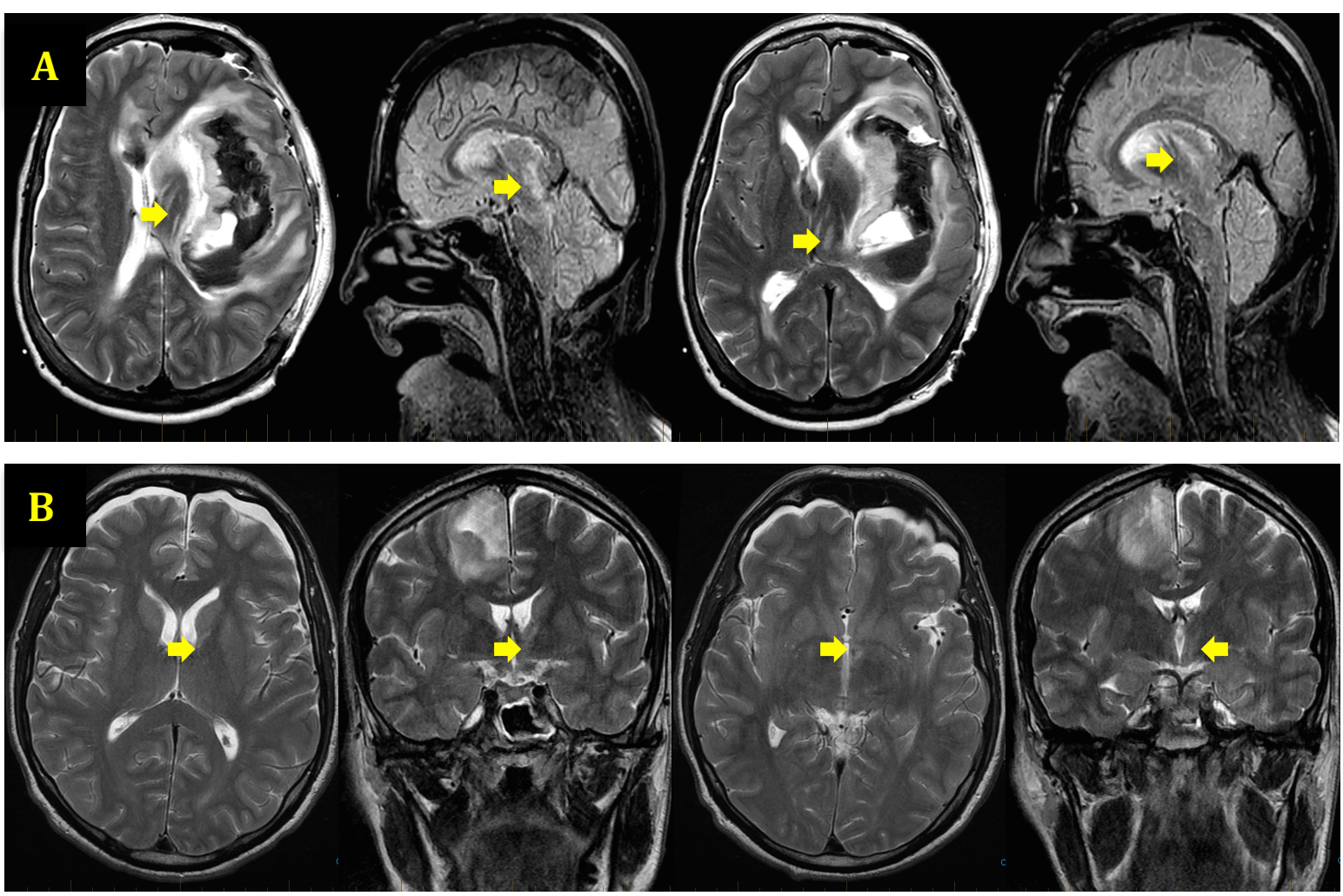

Magnetic resonance images (MRI) showing ventricular catheter misplacement and multiple passes morbidity in traumatic and non-traumatic examples (arrows). A: Middle age patient with spontaneous intracerebral hemorrhage. Axial and sagital MRI images showing multiple EVD tracts in the left thalamus down to the midbrain. At least three different catheter passes can be identified. B: Middle age with brain injury. Axial and coronal MRI images showing EVD tract in the left striatum down to subthalamic region. 
Figure 24: Head scan differences when using the CT EVD Protocol and routine axial scan
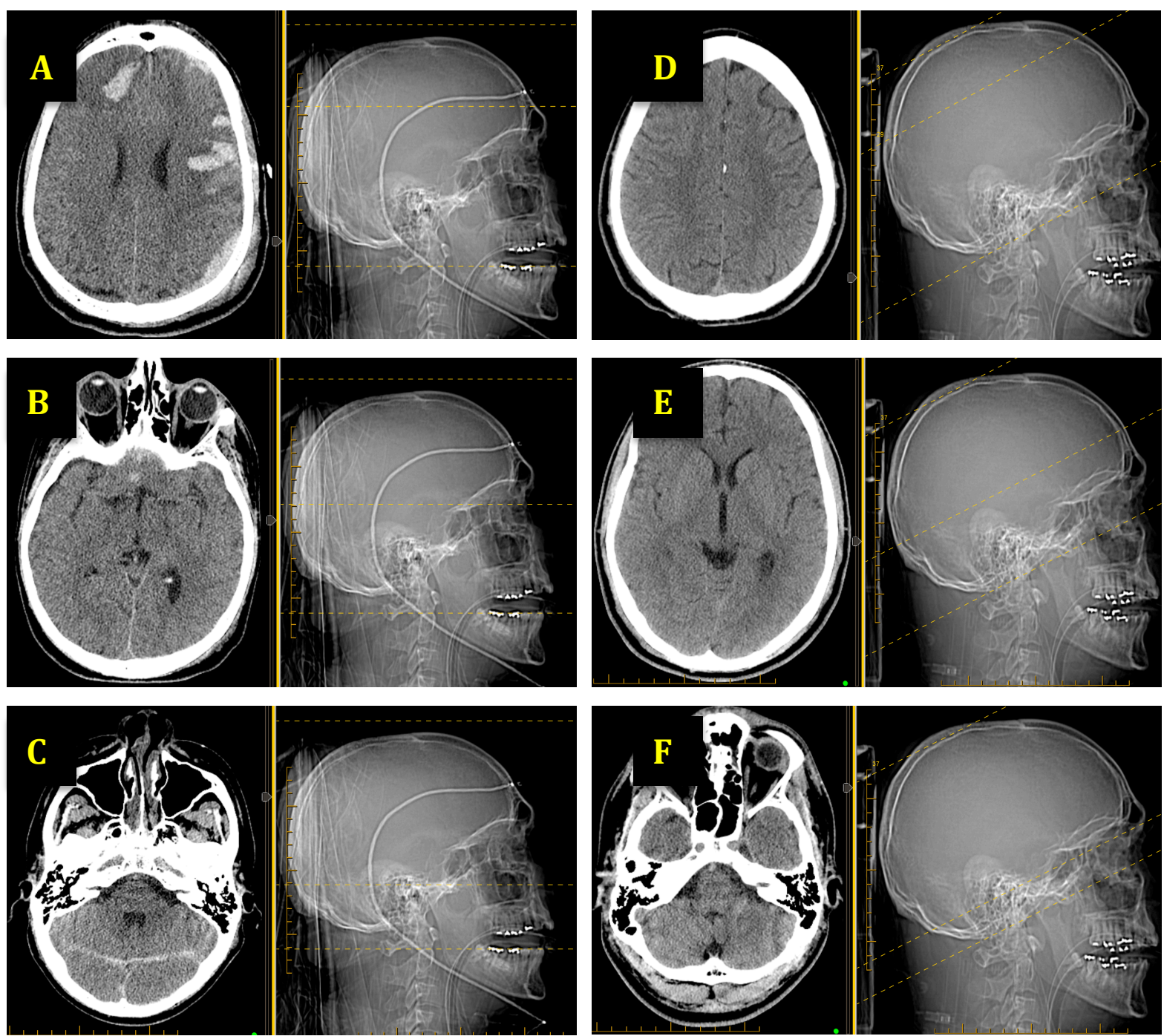

Computed tomography images; axial scans with their reference lines in the scout images, showing the difference between the two techniques corresponding to the gantry tilt. The navigation system requirement is a 0 tilt (A-C), which was used for the CT EVD Protocol. Routine axial head scans are done with a tilt leveled parallel to the orbitomeatal line (D-F). 
Figure 25: Example of the scanning pitfalls when used for the navigation guidance

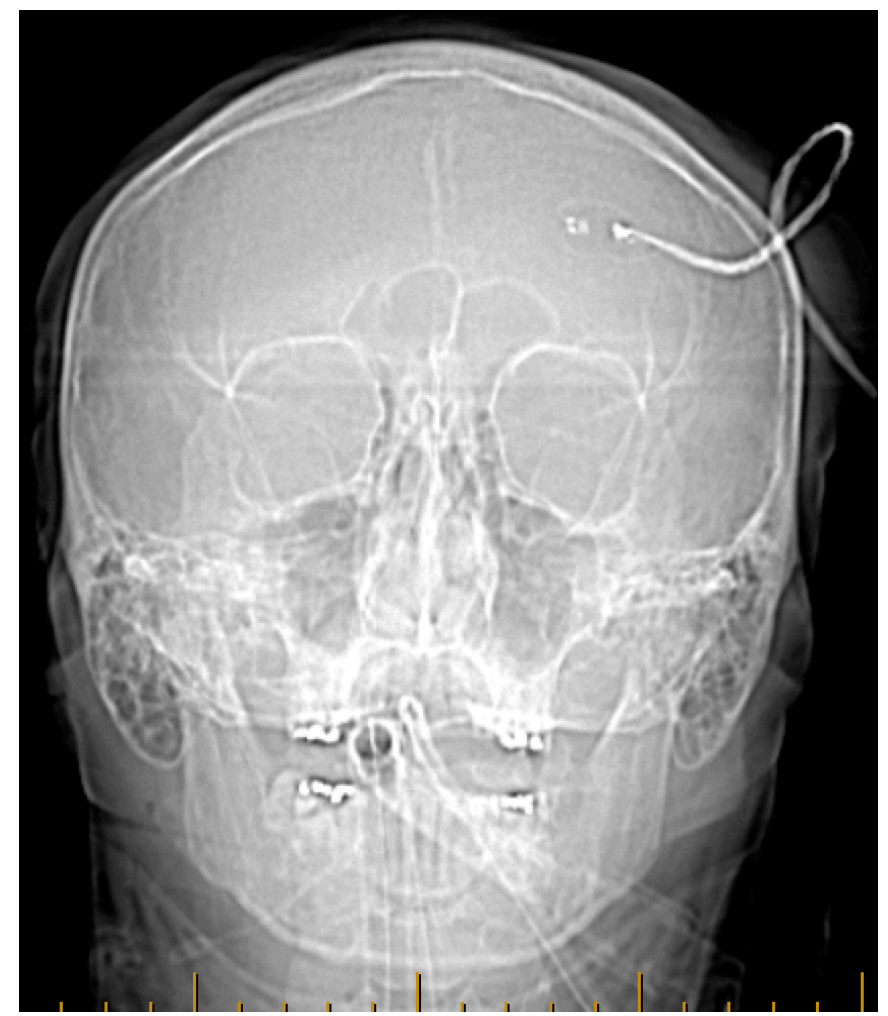

Scanning with objects on the forehead as oximetry probe, as in this example, contributing to difficulty in navigation registration. 
APPENDICES 
Appendix A: A table of the standard Glasgow Coma Scale (GCS) scores and severity

Table: Standard Glasgow Coma Scale (GCS) and Severity

\begin{tabular}{|l|l|l|}
\hline Eye opening & Best verbal response & Best motor response \\
\hline & 5: oriented & 6: obeys commands \\
4: spontaneous & 4: confused & 5: localizes \\
3: to speech & 3: inappropriate words & 4: withdraws \\
3: to pain & 2: incomprehensible sounds & 2: decortication \\
1: none & 1: none & 1: none \\
\hline Severity Score: & \\
\hline Total GCS score: 3 - 15 & \\
Mild TBI: GCS 13 - 15 & \\
Moderate TBI: GCS 9 - 12 & \\
Severe TBI: GCS 3 - 8 &
\end{tabular}

Traumatic brain injury (TBI)

Reference: Teasdale GG. Assessment of coma and impaired consciousness. A practical scale. Lancet, The. 1974;2(7872):81-4. 


\section{Appendix B: A table of the indications for intracranial pressure (ICP) monitoring in severe traumatic brain injury (TBI) patients}

Table: The indications for ICP monitoring in severe TBI according to the recommendations of the Brain Trauma Foundation:

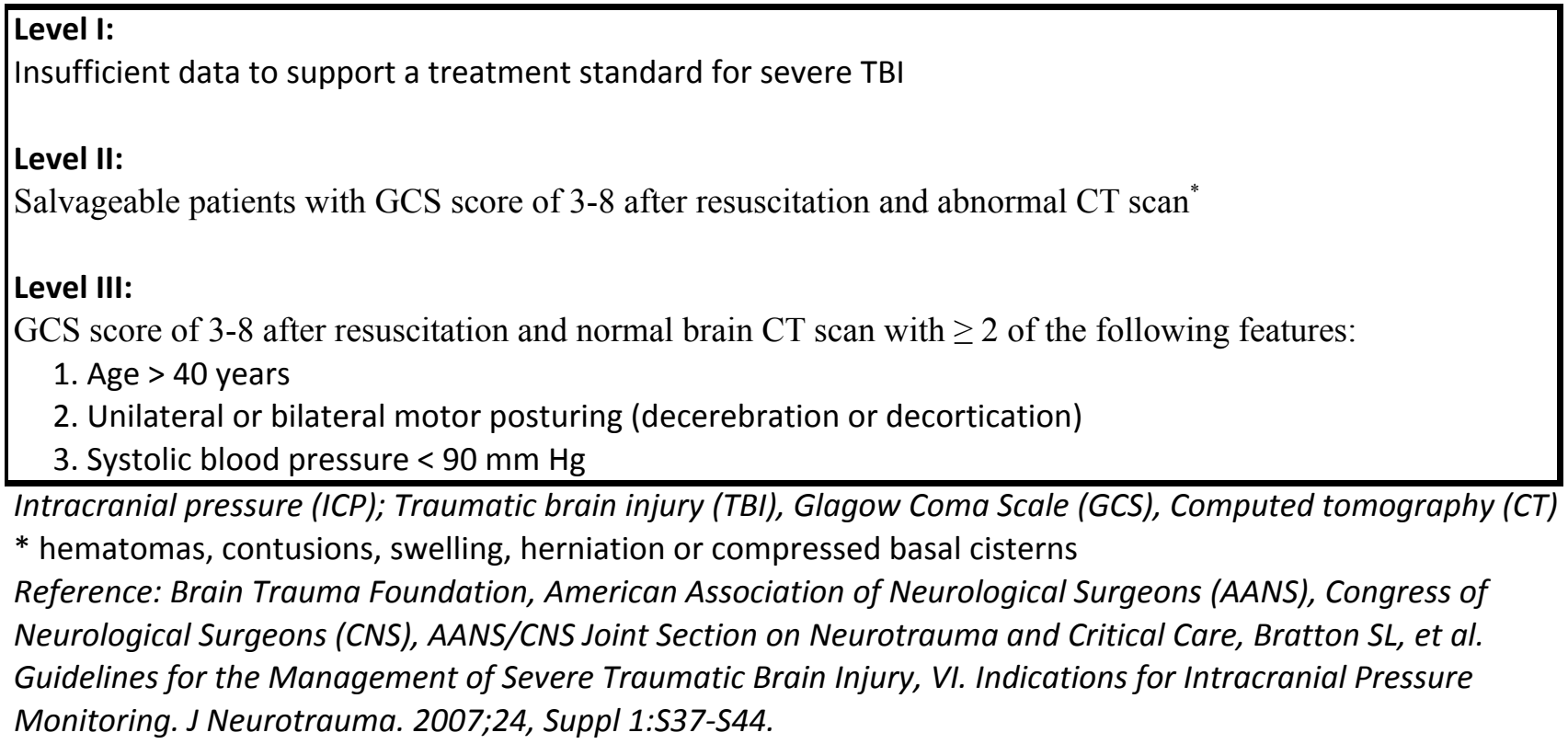




\section{Appendix C: A table of the Kakarla grading for the evaluation of the accuracy of ventricular catheter placement}

Table: Kakarla Grading System for the catheter tip location

\begin{tabular}{|l|l|l|}
\hline Grade & Accuracy of placement & Location of catheter tip \\
\hline 1 & Optimal/ adequate & $\begin{array}{l}\text { Ipsilateral frontal horn, including tip of } \\
\text { the third ventricle }\end{array}$ \\
\hline 2 & $\begin{array}{l}\text { Suboptimal (shallow) in } \\
\text { noneloquent tissue }\end{array}$ & $\begin{array}{l}\text { Contralateral frontal horn or lateral } \\
\text { ventricle, corpus callosum, } \\
\text { interhemispheric fissure }\end{array}$ \\
\hline 3 & $\begin{array}{l}\text { Suboptimal in eloquent } \\
\text { tissue }\end{array}$ & $\begin{array}{l}\text { Brainstem, cerebellum, internal capsule, } \\
\text { basal ganglia, thalamus, occipital cortex, } \\
\text { basal cisterns }\end{array}$ \\
\hline
\end{tabular}

Reference: Kakarla UK, Chang SW, Theodore N, Spetzler RF, Kim LJ. Safety and Accuracy of Bedside External Ventricular Drain Placement.

Neurosurgery. 2008;63:ONS162-ONS7. 


\section{Imaging Protocols}

for the Cranial, DBS, Spine, and ENT Applications

Technical Support Line

800.595.9709 or (+1) 720.890.3200

The following requirements apply to all scans taken for the Cranial, DBS, Spine, and ENT applications.

$\square$ Contrast agents may be injected before scanning.

$\square$ Scan contiguous slices.

$\square$ Use a constant slice thickness.

$\square$ Axial slices are preferred.

- CT scans: Axial/helical scans are acceptable using a pitch ratio of 1:1.

- MR scans: If 3D acquisition is used, reformat the scan to $1 \mathrm{~mm}$ axial slices.

$\square$ Use a circular (or square) field of view (FOV) - the smallest FOV to encompass the region of interest.

- Cranial and ENT scans: up to $25 \mathrm{~cm}(250 \mathrm{~mm})$ for a normal adult head size.

- Spine scans: up to $18 \mathrm{~cm}(180 \mathrm{~mm})$ to completely encompass all vertebrae of interest, including the spinous and transverse processes.

- Stereotactic scans: usually $28-32 \mathrm{~cm}(280 \mathrm{~mm}$ to $320 \mathrm{~mm})$ to include AC/PC structures, target region, entry region (if desired), and stereotactic frame (if applicable).

$\square$ Use a square image matrix of $256 \times 256$ or $512 \times 512$.

$\square$ Use square pixels.

$\square$ Archive the scan in an uncompressed file format (exception: compressed scans from GE scanners are acceptable).

$\square$ If you intend to merge two or more exams using StealthMerge ${ }^{\mathrm{TM}}$ or ENT Scan Merge software, ensure that several identifiable landmarks are included to enable matching.

\section{CT Scans Only}

$\square$ Standard soft tissue algorithm

$\square$ No gantry tilt

MR Scans Only

$\square$ No oblique slices

$\square$ Single-echo, no multi-echo scans

$\square$ Any MR imaging sequence can be used to show anatomy/pathology.

\section{Cranial and DBS Specific Requirements}

For Cranial and DBS scans only, observe the following additional requirements:

$\square$ Use a less than $3 \mathrm{~mm}$ slice thickness (any fractional slice thickness between 1 and $3 \mathrm{~mm}$ is acceptable).

- $1 \mathrm{~mm}$ slice thickness usually produces the highest quality dataset (depending on the scanner).

- $3 \mathrm{~mm}$ is the largest slice thickness acceptable for use with the Cranial application.

Note: Include the hard palate, tip of the nose, the ears, the top of the head, and all fiducial markers in the scan. For cranial scans, ensure that the tip of the nose is the most anterior point on the scan.

$\square$ Separate the patient's head from the scanner headrest with towels or foam.

9732379, rev. 5

$12 / 07$ 


\section{Appendix E: The correspondence with the medical physicist at the radiology department regarding the radiation dose safety of the new CT EVD Protocol}

\author{
"Claire Cohalan, Miss" <claire.cohalan@mcgill.ca> \\ "Claire Cehalan, Miss" \\ <claire.cohalan@mcgill.ca> \\ 07/03/2014 11:56 AM
}

\author{
To"jeffrexchankowsky@muhc.mcgillca" \\ <jeffrey.chankowsky@muhc.mcgill.ca>
}

$\mathrm{cc}$

SubjectCT dose for head trauma patients

Hi Jeff,

I looked at the potential dose for different head scans on the LightSpeed CT scanner (the left one in the CT department). I found that for a $\mathbf{2 . 5} \mathbf{~ m m ~ A x i a l , ~ f o r ~ a ~} 180 \mathrm{~mm}$ coverage, $120 \mathrm{kVh}, 320 \mathrm{~mA}$, it delivered a dose of 61.3 $\mathrm{mGy}$, which is an acceptable dose (a routine head should be between 50 and $80 \mathrm{mGy}$ ). Now this would change drastically depending on the collimator width. I was using a $20 \mathrm{~mm}$ width, taking $8 \times 2.5 \mathrm{~mm}$ slices at a time. Taking $16 \times 1.25 \mathrm{~mm}$ slices would result in the same dose, but would lead to a noisier image, unless the $\mathrm{mA}$ was changed. For $1.25 \mathrm{~mm}$ Axial, for a proper SNR, the protocol required $640 \mathrm{~mA}$, which lead to a dose of $126.2 \mathrm{mGy}$, which is too high. We had talked about a $2 \mathrm{~mm}$ Axial as well, but that is not possible on those machines, due to the physical size of the detectors.

Gyorgy and I had done a similar measurement, $2.5 \mathrm{~mm}$ Axial, on a patient on the other machine (the CT on the right), and it lead to a good image and $62 \mathrm{mGy}$ dose, which again is good.

Should we specify a trauma head protocol directly on the machines, to make sure that the proper image is taken, which leads to a reasonable dose?

If you want to talk about this, we can meet later today or next week. You can reach me on Gyorgy's phone (42875 he's on vacation next week as well), by email, or come by my office (C5-164).

Thanks,

Claire Cohalan

Medical Physicist(See attached file: pic24271.gif) 


\section{Appendix F: Electromagnetic AxiEM system equipment}
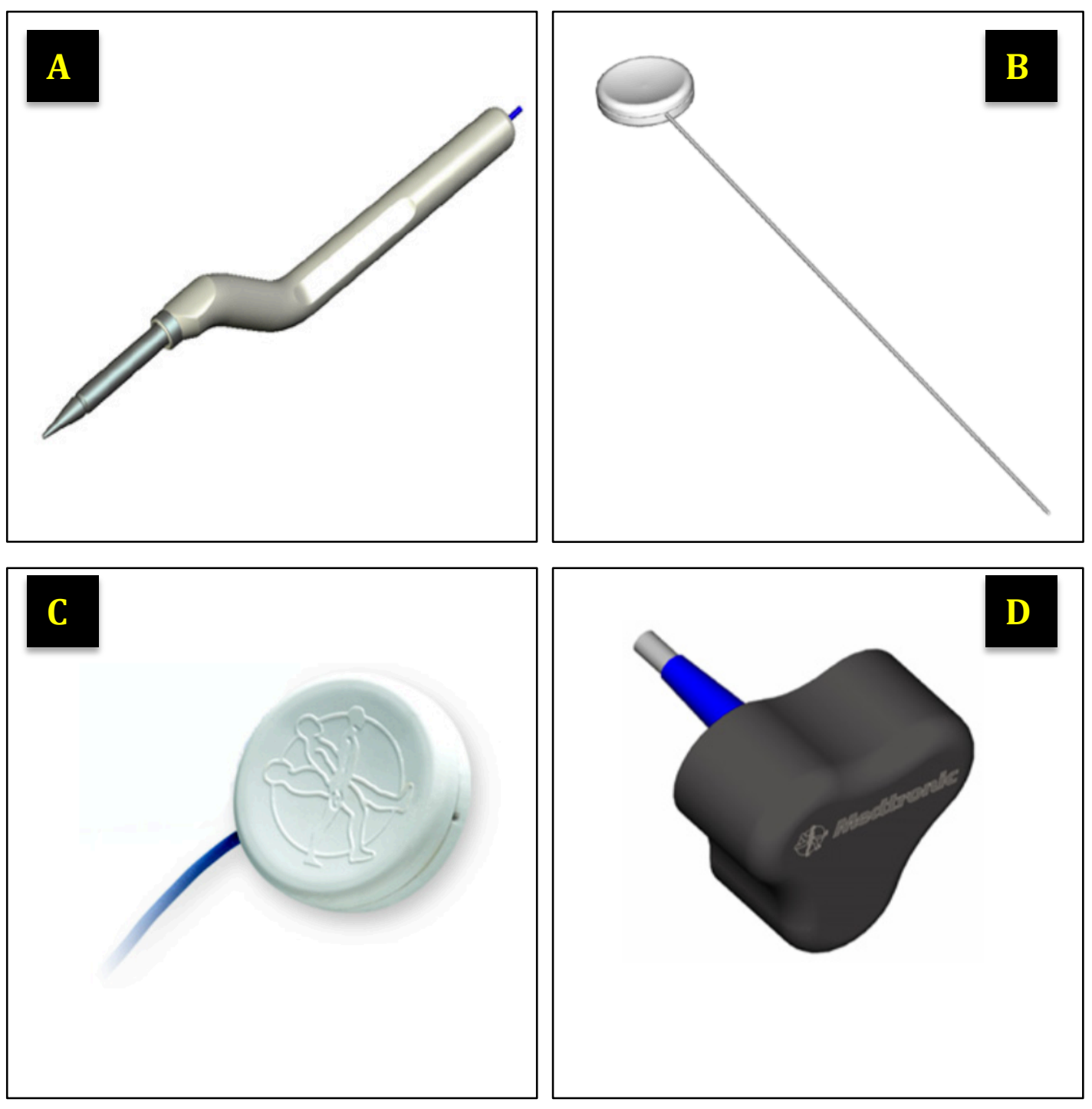

Standard equipment for the electromagnetic AxiEM system. A: Tracer pointer: Used to perform tracer registration. B: AxiEM stylet: A pointing device that can be used to place ventricular catheter. C: Non-invasive patient tracker: Acts as a continuous point of reference for the mobile emitter (rigidly positioned with respect to the patient's head). D: AxiEM mobile emitter: Encompasses the patient anatomy and the electromagnetic devices in a low-energy navigation field tracking their position within the field and relative to each other.

Reference: Medtronic Navigation Inc. Synergy Cranial AxiEM Pocket Guide.

Louisville: Medtronic Navigation; 2008, Revision 4, 01/2009. p. 10-54. 


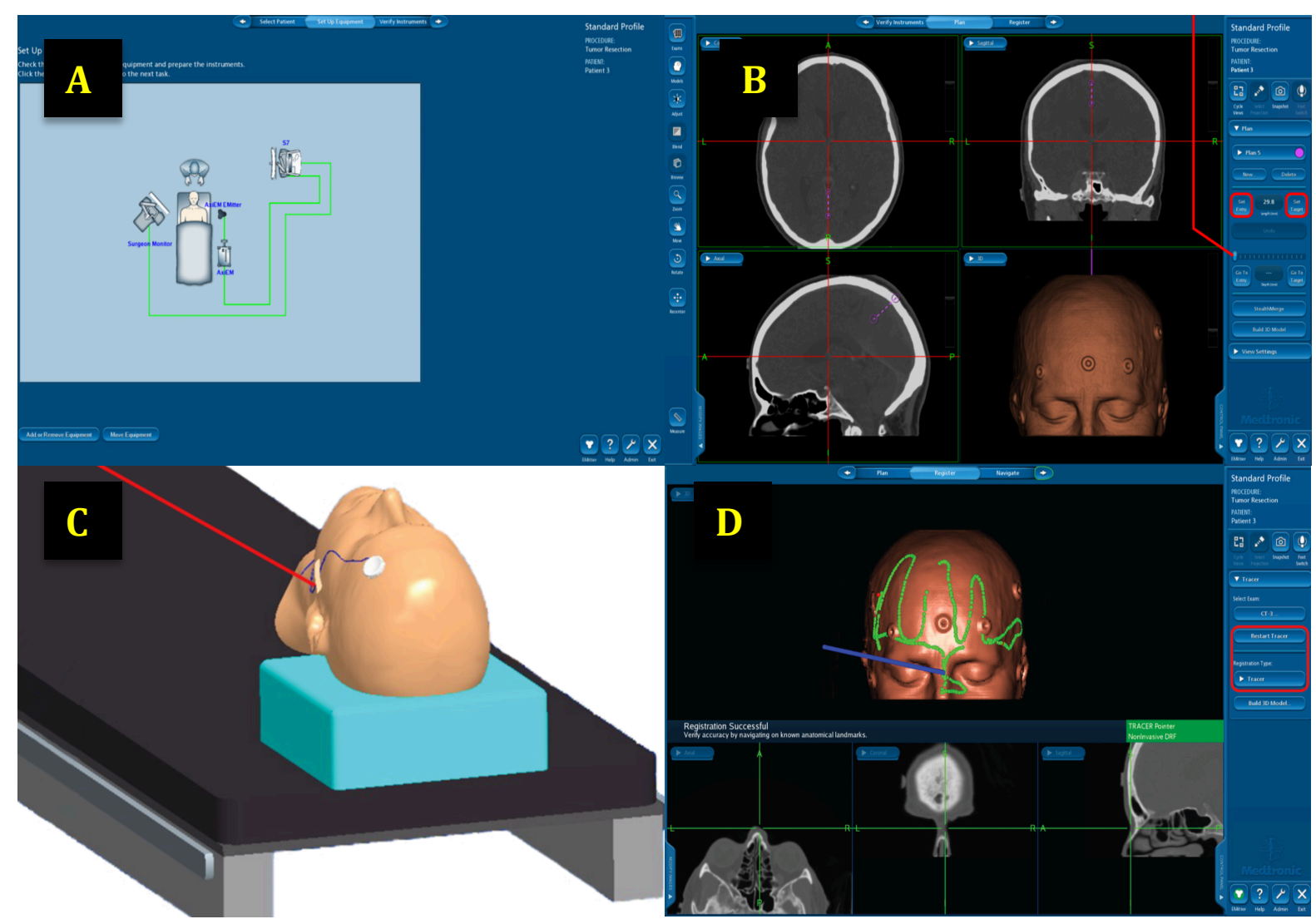

Electromagnetic navigation system setup, registration and navigation. A: The setup of the navigation system equipment. Green lines correspond to the connectivity between equipment. B: After building a 3-D model, target setup is done by clicking into the desired location. C: The way the non-invasive patient tracker is rigidly attached to the patient head and used as a reference for the mobile emitter. D: The registration using the surface tracing and verification of registration accuracy.

Reference: Medtronic Navigation Inc. Synergy Cranial AxiEM Pocket Guide. Louisville: Medtronic Navigation; 2008, Revision 4, 01/2009. p. 10-54. 
Appendix H: A table of the diffuse axonal injury (DAI) grades

Table: Diffue Axonal Injury Grades

\begin{tabular}{|l|l|}
\hline Grade 1 & $\begin{array}{l}\text { Histological evidence of axonal injury in the white matter of cerebral hemispheres, } \\
\text { the corpus callosum, the brain stem and the cerebellum. Radiologically, there are } \\
\text { lesions at grey-white matter interface. }\end{array}$ \\
\hline Grade 2 & Additionally, there is focal lesion in the corpus callosum \\
\hline Grade 3 & Additionlly, there is focal lesion in the dorsolateral aspect of the rostral brain stem \\
\hline
\end{tabular}

Adams JH. Diffuse axonal injury in head injury: definition, diagnosis and grading. Histopathology. 1989;15(1):49. 


\section{Appendix I: A table of the Marshall Grading for structural abnormalities in CT scan in TBI}

Table: Marshall Grading for structural abnormalities visualised in CT scan in TBI

\begin{tabular}{|c|c|c|}
\hline \multicolumn{2}{|c|}{ Grade } & Definition \\
\hline 1 & Diffuse Injury I (no visible pathology) & no visible intracranial pathology seen on CT scan \\
\hline \multirow[t]{4}{*}{ II } & \multirow[t]{4}{*}{ Diffuse Injury II } & cisterns are present with midline shift 0-5mm and/or; \\
\hline & & lesion densities present \\
\hline & & no high- or mixed-density lesion > $25 \mathrm{cc}$ \\
\hline & & may include bone fragments and foreign bodies \\
\hline \multirow[t]{2}{*}{ III } & \multirow[t]{2}{*}{ Diffuse Injury III (swelling) } & cisterns compressed or absent with midline shift $0-5 \mathrm{~mm}$, \\
\hline & & no high- or mixed-density lesion > $25 \mathrm{cc}$ \\
\hline \multirow[t]{2}{*}{ IV } & \multirow[t]{2}{*}{ Diffuse Injury IV (shift) } & midline shift $>5 \mathrm{~mm}$, \\
\hline & & no high- or mixed-density lesion > $25 \mathrm{cc}$ \\
\hline $\mathrm{V}^{\S}$ & Evacuated mass lesion & any lesion surgically evacuated \\
\hline $\mathrm{VI}^{\S}$ & Nonevacuated mass lesion & high- or mixed-density lesion > 25 cc, not surgically evacuated \\
\hline
\end{tabular}

Computerized tomogrpahy (CT); Traumatic Brain Injury (TBI).

$\S$ The Roman numerals $\mathrm{V} \& \mathrm{VI}$ were not in the original grading, and added here to make it better represented Lawrence F. Marshall, Sharon Bowers Marshall, Melville R. Klauber, Marjan van Berkum Clark, Howard M. Eisenberg, John A. Jane, et al. A new classification of head injury based on computerized tomography. Special Supplements. 1991;75(1s):S14-S20. 


\title{
Appendix J: Correspondence to Medtronic after mid interim analysis
}

March 06, 2015

Dr. Leslie Holton

External Research Program

Manager Medtronic Surgical Technologies

leslie.holton@medtronic.com

\author{
Re: EVD project using the neuronavigation system at the Intensive Care Unit of the Montreal General Hospital, McGill University Health Centre INTERIM \\ ANALYSIS
}

Dear Dr. Holton;

We are writing to you for an update regarding our research project, entitled

Placement accuracy of external ventricular drains (EVD) when comparing free-hand insertion to stereotactic neuronavigation-guided insertion at bedside in severe traumatic brain injury.

The study was officially launched in September 2014. While we did not receive the necessary disposable equipment supplies, including the Stylet and the Pointer kits, until October 21 2014, we managed to use some supplies purchased by the Montreal Neurological Hospital for clinical use while awaiting their delivery.

Owing to the technical requirements of neuronavigation, the Medtronic StealthStation AxiEM navigation system requires that imported patient imaging reach certain specifications, specifically without gantry tilt and adequately small slice thickness. We worked with the radiology department protocol at the Montreal General Hospital (MGH) to devise a computed tomography (CT) scan protocol for this study, which included a high-resolution axial scan with slice thickness of $2.5 \mathrm{~mm}$ and zero degree gantry tilt. All patients who present to the emergency room with a suspected diagnosis of traumatic brain injury are routinely imaged under this CT protocol. This means that all potential candidates have a CT compatible with the Medtronic StealthStation for neuronavigation and a subsequent, second CT is not required. This is important since it avoids further delay in patient treatment, and unnecessary exposure to radiation.

We completed a first interim analysis in mid-December 2014 involving the first 9 subjects recruited. Imaging of 9 subjects were compared to 9 control subjects and analyzed by a neuroradiologist, who was blinded to the method of insertion (with or without navigation), to assess the EVD placement. For the 9 navigation test subjects, we found that there was an accuracy of $100 \%$ in desired EVD tip location using the Kakarla grading system. The success from the first pass was in $8 / 9$ cases. None of the cases required further revision and there was a small subdural hematoma in one of the cases post EVD insertion. The procedure involves an additional time for the setup and registration when using the navigation. Unfortunately, it is difficult to quantify the actual additional time, as the retrospective group has no time recorded. Of note is that the procedure for each patient was urgent but not an extreme emergency meaning that it was necessary to complete the procedure within a short amount of time, i.e. one hour, and no patient had any adverse event related to the additional time for setup. In the control group without neuronavigation guidance, the accuracy of EVD tip location was $44 \%$, first pass success was in $5 / 7$, and revision was required in $44 \%$ of the cases.

It is also important to note that we were able to do all the procedures without the need for the neuronavigation personal available for the procedure. This is a key point as this procedure is urgent and needs to be done at any time of the day or the night, without hours of delay. Additionally, this can reduce its overall cost Though the results are promising, it is important to note that these are the interim analysis results and we cannot establish the significance at this early stage. We failed to register one patient, as there was ongoing facial and scalp swelling since the time of CT scan. Additionally, another patient was not enrolled as the CT scan was not done as per the protocol and we do not subject this group to another scan unless clinically indicated. This had happened at the beginning of the study and we followed it up to ensure optimum adherence to this protocol.

We are planning to finish the recruitment by this summer; however, the winter this year is unexpectedly slow with not much cases being recruited. The number of potential cases should go up in the spring and summer seasons.

We have made several observations about the system design and use within the intensive care setting, which may be useful to provide to you as feedback. There are few obstacles that we are facing with this system for EVD placement in the ICU. Mainly, as it is an electromagnetic field-based system, it was very difficult to use metal instruments in the field and it becomes complicated to make use of a self- retaining retractor that is often employed to provide better visualization for the wound opening and skull bone drilling. Additionally, we noted that the range of the electromagnetic field of the EM emitter is limited under $30 \mathrm{~cm}$ and within $20 \mathrm{~cm}$ for ideal usage. We also note that it is always worrisome to lose the patient Tracker during the procedure by accidental mobilization. This is especially important in trauma as almost all these patients have cervical collar, which can accidently push into the Tracker. Another problem that could be easily addressed is the organization of the disposables in the two kits, which is not ideal for trauma setting. The Stylet kit is the only piece that shall be maintained sterile throughout the procedure. We recommend that the Stylet be in a separate kit where the Tracker is added to the Pointer kit. Additionally, we think that the length of the Stylet is optimum, but we are facing a problem with it, as we have to cut the standard EVD tube short to be able to guide it. Though this system is compact, we believe it can be made even smaller and much easier to use. As an example, we could use a laptop instead of the system and adding the AxiEM Portable System to the same computer, so 3 pieces are made into one efficient piece. The last point to add is regarding the EMitter holder. We were unable to use it, as it was not planned for ICU beds, hence we were using traditional ways like using some cushions to support it. It is important to make a universal holder for the EMitter that can be used in different settings.

We will be glad to work with your team to get solutions for all these issues. And to be able to familiarize this procedure, we may ask you in future to provide workshops for the neurosurgery staff and residents plus the interested ones from trauma and intensive care unit (ICU). A future goal in using this system is that it is user-friendly and can be used in ICU in all trauma centers.

Sincerely;

Principal Investigator

Dr. Judith Marcoux

Montreal General Hospital

1650 Cedar Ave, Room L7-524

Montreal, QC

H3G 1 A4

Co-principal Investigator:

Dr. Ahmed AlAzri

Master student in Experimental Surgery

Co-principal Investigator:

Mr. Kelvin Mok Neuronavigation unit

Collaborator:

Dr. Jeff Chankowsky Neuroradiologist 